Pontifícia Universidade C Católica

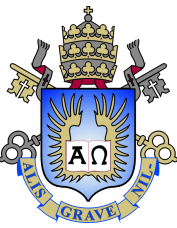

Matheus Secco Torres da Silva

Arithmetic structures in random sets

Tese de Doutorado

Thesis presented to the Programa de Pós-graduação em Matemática of PUC-Rio in partial fulfillment of the requirements for the degree of Doutor em Matemática.

Advisor: Prof. Simon Griffiths

Rio de Janeiro

July 2020 

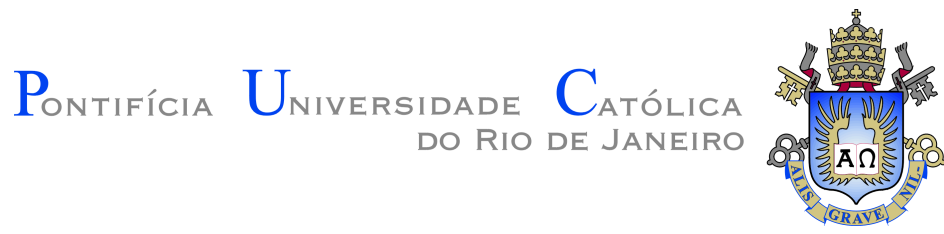

Matheus Secco Torres da Silva

\section{Arithmetic structures in random sets}

Thesis presented to the Programa de Pós-graduação em Matemática of PUC-Rio in partial fulfillment of the requirements for the degree of Doutor em Matemática. Approved by the undersigned Examination Committee.

Prof. Simon Griffiths

Advisor

Departamento de Matemática - PUC-Rio

Prof. Carlos Hoppen

Departamento de Matemática Pura e Aplicada - UFRGS

Prof. Edgard Pimentel

Departamento de Matemática - PUC-Rio

Prof. Maurício Collares Neto

Departamento de Matemática - UFMG

Prof. Robert Morris

Instituto de Matemática Pura e Aplicada - IMPA

Prof. Roberto Imbuzeiro Oliveira

Instituto de Matemática Pura e Aplicada - IMPA

Prof. Silvius Klein

Departamento de Matemática - PUC-Rio

Prof. Taísa Lopes Martins Instituto de Matemática e Estatística - UFF

Rio de Janeiro, July the 10th, 2020 
All rights reserved.

\section{Matheus Secco Torres da Silva}

Bachelor's degree in Mathematics by Pontifícia Universidade Católica do Rio de Janeiro (PUC-Rio) in 2014. Master degree in Mathematics by Fundação Getúlio Vargas (FGV) in 2016. Research interests include extremal and probabilistic combinatorics, as well as random processes and Ramsey theory.

Bibliographic data

Secco Torres da Silva, Matheus

Arithmetic structures in random sets / Matheus Secco Torres da Silva; advisor: Simon Griffiths. - Rio de Janeiro: PUC-Rio, Departamento de Matemática, 2020.

v., 87 f: il. color. ; $30 \mathrm{~cm}$

Tese (doutorado) - Pontifícia Universidade Católica do Rio de Janeiro, Departamento de Matemática.

Inclui bibliografia

1. Matemática - Teses. 2. Combinatória - Teses. 3. Probabilidade - Teses. 4. Desvios moderados;. 5. Martingais;. 6. Hipergrafos;. 7. Progressões aritméticas;. 8. Processos aleatórios.. I. Griffiths, Simon. II. Pontifícia Universidade Católica do Rio de Janeiro. Departamento de Matemática. III. Título. 
To my wife, my parents and my relatives, for their support and encouragement. 


\section{Acknowledgments}

I would like to thank you all my family, specially my parents, Helcio and Carla, my grandparents, Antônio, Lordes, Helcio and Marcia, my brothers, Ian, Iasmin and Mayara and also my uncle, Ricardo, that introduced me to the beautiful world of Mathematics.

The support of my wife Camilla was also imperative in these four years of hard working. Thank you, my love!

I would also like to thank you my advisor Simon for all conversations and for all the support. I will miss working with you but I am sure that we will still work together in the future. Thank you, Simon!

I would also like to thank you the professors Rob Morris, Yuri Lima and Nicolau Saldanha for writing recommendation letters for me that helped a lot in my job hunt.

I would also like to thank you the professors that I had in the Math olympiads. Thank you, Fabinho, Luciano, Samuel, Yuri, Gugu and Alex! I would also like to thank you two friends that I made in this path, Hugo and Renan. You are like brothers to me!

I would also like to thank you the friends that were together with me at PUC-Rio. Thank you, Igor, David, Daniel, Byron, Luize, Handel, José and Leonardo.

I would also like to thank my undegrad advisor (Ricardo Sá Earp) and my master advisor (Vincent Guigues). These acknowledgments extend to all professor that I have had at PUC-Rio and FGV.

This study was financed in part by the Coordenação de Aperfeiçoamento de Pessoal de Nível Superior - Brasil (CAPES) - Finance Code 001. 


\section{Abstract}

Secco Torres da Silva, Matheus; Griffiths, Simon (Advisor). Arithmetic structures in random sets. Rio de Janeiro, 2020. 87p. Tese de doutorado - Departamento de Matemática, Pontifícia Universidade Católica do Rio de Janeiro.

In this Ph.D. thesis, we study bounds for the deviation probabilities of a random variable $X$ that counts the number of edges of a hypergraph induced by a random $m$-element subset of its vertex set. We consider two contexts: the first corresponds to hypergraphs with some kind of regularity, whereas the second addresses hypergraphs that are in some sense far from being regular. It is possible to apply these results to discrete structures such as the set of $k$-term arithmetic progressions in the additive group of integers modulo a prime and in the set of the first $N$ positive integers. Furthermore, we also deduce results for the case when the random subset is generated by including each vertex of the hypergraph independently with probability $p$.

\section{Keywords}

Moderate deviations; Martingales; Hypergraphs; Arithmetic progressions; Random processes. 


\section{Resumo}

Secco Torres da Silva, Matheus; Griffiths, Simon. Estruturas aritméticas em conjuntos aleatórios. Rio de Janeiro, 2020. 87p. Tese de Doutorado - Departamento de Matemática, Pontifícia Universidade Católica do Rio de Janeiro.

Nesta tese de Doutorado, nós estudamos cotas para as probabilidades de desvio de uma variável aleatória $X$ que conta o número de arestas de um hipergrafo induzido por um subconjunto aleatório de $m$ elementos do seu conjunto de vértices. Nós consideramos dois contextos: o primeiro corresponde a hipergrafos que possuem certo tipo de regularidade, ao passo que o segundo lida com hipergrafos que são, em algum sentido, longe de serem regulares. É possível aplicar estes resultados a estruturas discretas, como o conjunto de progressões aritméticas de tamanho $k$ no grupo aditivo de inteiros módulo um primo e também no conjunto dos $N$ primeiros inteiros positivos. Além disso, também deduzimos resultados para o caso em que o subconjunto aleatório é gerado incluindo cada vértice do hipergrafo independentemente com probabilidade $p$.

\section{Palavras-chave}

Desvios moderados; Martingais; Hipergrafos; Progressões aritméticas; Processos aleatórios. 


\section{Table of contents}

1 Introduction $\quad 11$

1.1 Description of the main results 13

1.2 Layout of the thesis 19

2 Preliminaries $\quad 21$

2.1 Basic notations 21

2.2 Asymptotic notations 21

2.3 Hypergraphs 22

$\begin{array}{ll}2.4 & \text { Conditional expectation and Martingales } \\ 2.5 & 25\end{array}$

2.5 Martingale concentration inequalities 27

2.6 Estimates for the binomial distribution 30

3 General setup of the problem 31

3.1 Notations 31

3.2 Martingale representation $\quad 32$

$\begin{array}{lll}\text { 3.3 Some useful probability results } & 34\end{array}$

4 Deviations in m-model for regular hypergraphs $\quad 36$

4.1 A deterministic control of the increments 38

4.2 The base case 38

4.3 $P_{r}$ implies $Q_{r} \quad 39$

4.4 $Q_{r}$ implies $P_{r+1} \quad 42$

5 Deviations in p-model for regular hypergraphs $\quad 45$

5.1 Lower Bound 47

$\begin{array}{ll}5.2 & \text { Upper Bound }\end{array}$

6 Deviations in m-model for non-regular hypergraphs $\quad 51$

6.1 Approximation of the increments 52

6.2 Control of the error term 57

6.3 Approximation of $\Lambda^{\mathcal{H}}\left(B_{m}\right) \quad 59$

6.4 Conditional variance of $\Lambda^{\mathcal{H}, *}\left(B_{m}\right) \quad 61$

6.5 Deviations for $\Lambda^{\mathcal{H}, *}\left(B_{m}\right) \quad 65$

6.6 Proof of Theorem 6.1 68

$\begin{array}{lll}7 & \text { Deviations in p-model for non-regular hypergraphs } & 71\end{array}$

8 Applications for arithmetic configurations in random sets $\quad \mathbf{7 5}$

8.1 Progressions in $\mathbb{Z}_{N} \quad 75$

8.2 Schur equation in $\mathbb{Z}_{N} \quad 77$

8.3 Sidon equation in $\mathbb{Z}_{N} \quad 77$

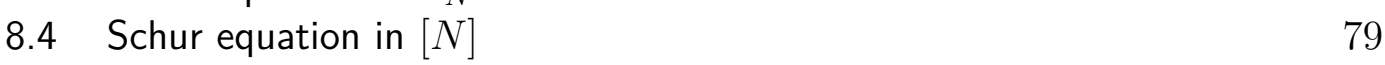

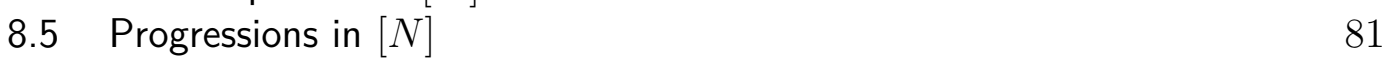

$\begin{array}{lr}\text { Bibliography } & 85\end{array}$ 


\section{List of figures}

Figure 1.1 The result of Bhattacharya, Ganguly, Shao and Zhao [21] applies in the blue and the green regions. On the other hand, Corollary 1.6 applies in the green and the red regions.

Figure 1.2 The result of Bhattacharya, Ganguly, Shao and Zhao [21] applies in the blue and the green regions. On the other hand, Corollary 1.6 applies in the green and the red regions.

Figure 4.1 Illustration of the sequence of implications. 
Mathematics is the surest way to immortality. If you make a big discovery in mathematics, you will be remembered after everyone else will be forgotten.

Paul Erdős, The Magician of Budapest. 


\section{Introduction}

In this thesis, we study how the number of $k$-term arithmetic progressions (and other discrete structures) in a random set deviates from its mean.

A $k$-term arithmetic progression ( $k$-AP for short) is a sequence of the form $(a, a+d, a+2 d, \ldots, a+(k-1) d)$, where $a$ and $d$ are integers (we also consider the case where $a$ and $d$ are elements of the additive group modulo $\left.n, \mathbb{Z}_{n}\right)$. We remark that we do not consider trivial progressions $(a, \ldots, a)$ and when counting, we regard the progression $\left(a_{1}, a_{2} \ldots, a_{k}\right)$ as the same as $\left(a_{k}, a_{k-1}, \ldots, a_{1}\right)$.

One of the first results involving arithmetic progressions in Combinatorics was obtained by van der Waerden in 1927 [1], who proved that every coloring of the natural numbers with a finite number of colors contains arbitrarily long monochromatic APs. In 1936, Erdős and Turán [2] conjectured that every subset of $\mathbb{N}$ with positive upper density contains $k$-APs for all lengths $k$. This conjecture was proved by Roth in 1953 [3] for $k=3$, using Fourier methods. In 1969 [4], Szemerédi extended the result for $k=4$ and in 1975 [5], Szemerédi proved the full conjecture. The techniques used by Szemerédi culminated in one of the most powerful tools in extremal graph theory nowadays, the celebrated Szemerédi's regularity lemma.

Erdös also conjectured that any subset $A$ of $\mathbb{N}^{*}$ with $\sum_{a \in A} 1 / a=\infty$ must contain arbitrarily long APs. This conjecture was proven by Green and Tao [6] for the set of prime numbers, but it is still open in the general case, even for $3-\mathrm{APs}$.

On the other hand, one problem of great interest in Probabilistic Combinatorics is to understand the behavior of a random variable $X$ that counts the number of occurrences of some structure in a random set. There are many questions that can be asked for $X$. For example, one may ask if $X$ satisfies a law of large numbers or if $X$ converges in distribution to some random variable. In this work, we will focus on understanding how $X$ deviates from its mean, $\mathbb{E}[X]$.

This problem was widely studied for the cases where $X$ counts the number of copies of a fixed graph $H$ in a random graph and where $X$ counts the number of $k$-arithmetic progressions in a random set. In both cases, there are three 
types of deviations of interest: small deviations (of the order of the standard deviation of $X$ ), large deviations (of the order of the mean of $X$ ) and the intermediate range of moderate deviations.

In the case of deviations of subgraph counts in random graphs, Ruciński [7] proved that under some conditions on $p$, the number of copies of a fixed graph $H$ in the Erdős-Rényi random graph $G(n, p)$ is asymptotically normally distributed. This has been followed by some quantitatively stronger results $[8,9,10]$.

Regarding large deviations, general (but far from tight) bounds follow from the famous inequality of Kim and $\mathrm{Vu}[11]$. Important subsequent advances include the translation of such deviation problems into variational problems for graphons (Chaterjee and Varadhan [12]) and solutions to these variational problems for certain values of the parameters (Lubetzky and Zhao [13]). One may also consult the survey of Chatterjee [14] and the references therein for a more detailed overview. Very recently, Harel, Mousset, and Samotij [15] have greatly extended the range of such large deviations for graphs $H$ with some conditions.

For moderate deviations, much less is known. The first results of moderate deviations in $G(n, p)$ were obtained by Döring and Eichelsbacher [16, 17]. Very recently, Goldschmidt, Griffiths, and Scott [18] obtained asymptotic results for the rate associated with moderate deviations of subgraph counts in the Erdős-Rényi random graph $G(n, m)$ and as a byproduct, they also obtained asymptotic results for the Erdös-Rényi random graph $G(n, p)$. Their approach is based on a martingale representation of the subgraph count deviations, which we will extend in this thesis for the more general setup of hypergraphs.

In the case of $k$-APs in a random set obtained by choosing each element of $[N]:=\{1, \ldots, N\}$ independently with probability $p$, Janson and Ruciński [19] determined (under certain conditions) the log probability $\log (\mathbb{P}(X>(1+\delta) \mathbb{E}[X]))$, where $\delta$ is a constant, up to a factor of order $\log (1 / p)$. Following this work, Warnke [20] determined the log probability up to a constant factor (in which the constant may depend on $\delta$ ). Both works were developed in the more general context of randomly induced subhypergraphs. In the specific case of arithmetic progressions, Bhattacharya, Ganguly, Shao, and Zhao [21] have made further progress, obtaining the asymptotic of the log probability for a restricted range of $p$. They proved that

$$
\mathbb{P}(X \geqslant(1+\delta) \mathbb{E}[X])=p^{(1+o(1)) \sqrt{\delta} N p^{k / 2}}
$$

whenever $p \geqslant N^{-1 / 6 k(k-1)}(\log N)^{1 / k}$ and $p \rightarrow 0$. 
Recently, this question has been answered almost completely for large deviations (fixed $\delta>0$ ). In a recent breakthrough, Harel, Mousset, and Samotij [15] have proved that (1-1) holds across the whole range of densities $(\log N / N)^{2 / k} \ll p \ll 1$.

In this thesis, we address the problem of moderate deviations in the counting of $k$-APs in a random set obtained from both the set of the first $N$ positive integers, $[N]$, and from the additive group $\mathbb{Z}_{N}$, where $N$ is a prime number. We study this problem under the more general framework of hypergraphs and we prove general results for some classes of hypergraphs, from which we can deduce results for the counting of $k$-APs and other discrete structures, such as the number of solutions of the Schur equation and the Sidon equation.

\section{1}

\section{Description of the main results}

The general framework in which we can view these counting problems is the following: Given a hypergraph $\mathcal{H}$ on $[N]:=\{1, \ldots, N\}$ and a distribution on subsets $B \subseteq[N]$ we may ask how the random variable

$$
X:=e(\mathcal{H}[B])
$$

which represents the number of edges of $\mathcal{H}$ contained in $B$, is concentrated around its mean.

Our main focus is on the case where $B \sim B_{m}$ is a uniformly random $m$ element subset of $[N]=\{1, \ldots, N\}$. We prove upper bounds on the probability of deviations of the random variable $e\left(\mathcal{H}\left[B_{m}\right]\right)$ in two contexts: the first is when the hypergraph $\mathcal{H}$ is highly regular, which corresponds to arithmetic structures in $\mathbb{Z}_{N}$, with $N$ prime. This is based on joint work with Gonzalo Fiz Pontiveros, Simon Griffiths, and Oriol Serra [22].

The second context is when the hypergraph $\mathcal{H}$ is in some sense far from being regular, which is suitable for some discrete structures in $[N]$. Indeed, in this case, we may obtain asymptotic results for the log probability of moderate deviations. This is based on joint work with Simon Griffiths and Christoph Koch.

To illustrate these differences, let us consider the case $N=7$. The hypergraph corresponding to 3 -APs in $\mathbb{Z}_{7}$ is regular in the sense that each vertex lies in 9 edges. On the other hand, the hypergraph corresponding to 3 -APs in [7] is such that the vertices 1,2,6 and 7 belong to 4 edges, whereas the other vertices lie in 5 edges. 
Furthermore, since $B_{p}$, the $p$-random subset, may be obtained by averaging over the models $B_{m}$ where $m$ is selected according to $\operatorname{Bin}(N, p)$, the binomial distribution with parameters $N$ and $p$, in both cases we may deduce results in the context of $p$-random sets.

The following setup is common to both cases. Given a $k$-uniform hypergraph $\mathcal{H}$ on the vertex set $[N]$ and a subset $B \subseteq[N]$ we set

$$
N^{\mathcal{H}}(B):=e(\mathcal{H}[B]),
$$

the number of edges of $\mathcal{H}$ contained in the set $B$. We also define

$$
L^{\mathcal{H}}(m):=\mathbb{E}\left[N^{\mathcal{H}}\left(B_{m}\right)\right]
$$

the expected value of $N^{\mathcal{H}}\left(B_{m}\right)$ where $B_{m}$ is a uniformly selected $m$ element subset of $[N]$. Our focus will be on studying

$$
D^{\mathcal{H}}\left(B_{m}\right):=N^{\mathcal{H}}\left(B_{m}\right)-L^{\mathcal{H}}(m)
$$

the deviation of $N^{\mathcal{H}}\left(B_{m}\right)$ from its mean.

We now discuss the results obtained in the regular case. We say that a hypergraph $\mathcal{H}$ is $r$-tuple-regular ${ }^{1}$ if all $r$-tuples of vertices are included in the same number of edges. Note that all hypergraphs are 0-tuple-regular, and a hypergraph is 1-tuple-regular if it is regular in the usual sense that each vertex is in the same number of edges. We also note that, by a simple double counting argument, $r$-tuple regular implies $r^{\prime}$-tuple regular for all $0 \leqslant r^{\prime} \leqslant r$.

Theorem 1.1 Let $1 \leqslant r \leqslant k$. Let $\mathcal{H}$ be a $k$-uniform hypergraph on $[N]$. Suppose that $\mathcal{H}$ is $(r-1)$-tuple-regular with maximum $r$-degree $\Delta_{r}$. Then

$$
\mathbb{P}\left(D^{\mathcal{H}}\left(B_{m}\right)>a\right) \leqslant N^{O_{k}(1)} \exp \left(\frac{-\Omega_{k}(1) a^{2 / r}}{m \Delta_{r}^{2 / r}}\right)
$$

for all $a>0$.

Furthermore, the same bounds apply to the corresponding negative deviations.

Unfortunately some natural hypergraphs are not precisely $r$-tupleregular, but just very close to being so. Let $\bar{d}_{r}=\bar{d}_{r}(\mathcal{H})$ denote the average degree of $r$-sets in a hypergraph $\mathcal{H}$. We say that $\mathcal{H}$ is $(r, \eta)$-near-regular if every $r$-tuple of vertices is contained in $(1 \pm \eta) \bar{d}_{r}$ edges $^{2}$. Obviously, a hyper-

${ }^{1}$ Equivalently, in the language of combinatorial designs, $\mathcal{H}$ is an $r-(N, k, \lambda)$ design, for some $\lambda$.

${ }^{2}$ We write $x=a \pm b$ to express that $x$ belongs to the interval $[a-b, a+b]$. 
graph which is $r$-tuple-regular is $(r, \eta)$-near-regular for all $\eta \geqslant 0$. In particular, Theorem 1.1 is a special case $(\eta=0)$ of the following theorem.

Theorem 1.2 Let $1 \leqslant r \leqslant k$ and let $\eta \in\left[0,3^{-r+1}\right]$. Let $\mathcal{H}$ be a $k$-uniform hypergraph on $[N]$. Suppose that $\mathcal{H}$ is $(r-1, \eta)$-near-regular with maximum $r$-degree $\Delta_{r}$. Then

$$
\mathbb{P}\left(D^{\mathcal{H}}\left(B_{m}\right)>a\right) \leqslant N^{O_{k}(1)} \exp \left(\frac{-\Omega_{k}(1) a^{2 / r}}{m \Delta_{r}^{2 / r}}\right)
$$

for all

$$
a \geqslant(10 k !)^{10^{r}} e(\mathcal{H})\left(\frac{\eta m^{k-1}}{N^{k-1}}\right)^{r /(r-1)} .
$$

Furthermore, the same bounds apply to the corresponding negative deviations.

Remark 1.3 In the dense case $(m=\Theta(N))$, the bound given by Theorem 1.2 is best possible (up to the constant implicit in $\Omega_{k}(1)$ ) for all $r \geqslant 0$ and across the whole range $\Theta(1) \leqslant \Delta_{r} \leqslant \Theta(N)$. This is discussed in Section 6 of [22].

Remark 1.4 One may easily observe by a double counting argument that an $(r-1, \eta)$-near-regular hypergraph $\mathcal{H}$ is $\left(r^{\prime}-1, \eta\right)$-near-regular for all $1 \leqslant r^{\prime} \leqslant r$. So one may choose which of the above inequalities to apply. Therefore

$$
\mathbb{P}\left(D^{\mathcal{H}}\left(B_{m}\right)>a\right) \leqslant N^{O_{k}(1)} \min _{1 \leqslant r^{\prime} \leqslant r}\left\{\exp \left(\frac{-\Omega_{k}(1) a^{2 / r^{\prime}}}{m \Delta_{r^{\prime}}^{2 / r^{\prime}}}\right)\right\} .
$$

It is worth remarking that the minimum is not always obtained at the extremes $r^{\prime} \in\{1, r\}$, see the application to arithmetic progressions in $\mathbb{Z}_{N}$ in Section 8.1 for example.

When $B \sim B_{p}$ is a set where each element is independently chosen from $[N]$ with probability $p$, which may be as usual a function of $N$, we obtain the following result, where $L^{\mathcal{H}}(p)=\mathbb{E}\left[N^{\mathcal{H}}\left(B_{p}\right)\right]$ and $D^{\mathcal{H}}\left(B_{p}\right)=N^{\mathcal{H}}\left(B_{p}\right)-L^{\mathcal{H}}(p)$.

Theorem 1.5 Let $k \geqslant r \geqslant 2$. Let $\mathcal{H}_{N}$ be a sequence of $k$-uniform hypergraphs which are $(r-1, \eta)$-near-regular with maximum $r$-degree $\Delta_{r}$ and $V\left(\mathcal{H}_{N}\right)=[N]$. Let $\delta_{N}$ be a sequence satisfying $\max \left\{\frac{\Delta_{r}(N \log N)^{r / 2}}{p^{k-r / 2} e(\mathcal{H})},\left(\eta^{r} p^{k-r}\right)^{1 /(r-1)}, \frac{1}{\sqrt{p N}}\right\} \ll \delta_{N} \ll\left(\frac{p^{k-r} e(\mathcal{H})}{N^{r} \Delta_{r}}\right)^{1 /(r-1)}$, where $p \leqslant 1$ is bounded away from $1^{3}$. Then

$$
\mathbb{P}\left(D^{\mathcal{H}_{N}}\left(B_{p}\right)>\delta_{N} L^{\mathcal{H}_{N}}(p)\right)=\exp \left(-(1+o(1)) \frac{\delta_{N}^{2} p N}{2 k^{2}(1-p)}\right) .
$$

${ }^{3}$ This means that there exists a constant $\varepsilon>0$ such that $p \leqslant 1-\varepsilon$. 
Furthermore, the same bounds apply to the corresponding negative deviations.

As an immediate application, consider 3-term arithmetic progressions in the cyclic group $\mathbb{Z} / N \mathbb{Z}$, for $N$ prime. Let us write $D^{3}\left(B_{p}\right)$ for the deviation of the 3 -progressions count in a $p$-random subset $B_{p}$ of $\mathbb{Z} / N \mathbb{Z}$. Note that the expected number of such arithmetic progressions is $L^{3}(p)=p^{3}\left(\begin{array}{c}N \\ 2\end{array}\right)$. Since the hypergraph associated with 3 -APs in $\mathbb{Z}_{N}$ is 1 -regular with $\Delta_{2}=O(1)$ and has $\Theta\left(N^{2}\right)$ edges, Theorem 1.5 with $r=2$ and $\eta=0$ gives us the following result.

Corollary 1.6 Let $\delta_{N}$ be a sequence satisfying

$$
\max \left\{\frac{\log N}{p^{2} N}, \frac{1}{\sqrt{p N}}\right\} \ll \delta_{N} \ll p .
$$

If $p$ is bounded away from 1 , then

$$
\mathbb{P}\left(D^{3}\left(B_{p}\right)>\delta_{N} L^{3}(p)\right)=\exp \left(-(1+o(1)) \frac{\delta_{N}^{2} p N}{18(1-p)}\right) .
$$

Furthermore, the same bounds apply to the corresponding negative deviations.

Bhattacharya, Ganguly, Shao and Zhao [21] have proved that (1-2) holds under the following conditions on $p$ and $\delta_{N}: p \rightarrow 0, \delta_{N}=O(1)$, $\delta_{N}^{-3} p(\log (1 / p))^{2} \rightarrow \infty$, and

$$
\min \left\{\delta_{N} p^{3}, \delta_{N}^{2} p\right\} \geqslant N^{-1 / 6}(\log N)^{7 / 6}
$$

We observe that Corollary 1.6 extends in some sense their result. Indeed, let us consider for simplicity the cases $p=N^{-\gamma}$ and $\delta_{N}=N^{-\theta}$, where $\gamma, \theta>0$.

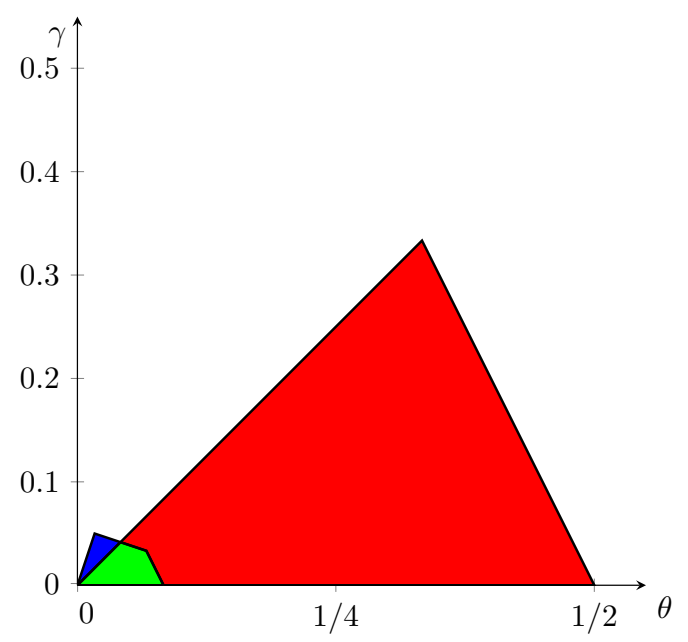

Figure 1.1: The result of Bhattacharya, Ganguly, Shao and Zhao [21] applies in the blue and the green regions. On the other hand, Corollary 1.6 applies in the green and the red regions. 
We now discuss the results obtained in the non regular case. In contrast to the near-regular cases considered first, the variance of the degrees in $\mathcal{H}$ now plays a central role. For a vertex $i \in[N]$ we write $d_{\mathcal{H}}(i)$ for the degree of $i$ in $\mathcal{H}$ so that

$$
\bar{d}(\mathcal{H}):=\frac{1}{N} \sum_{i=1}^{N} d_{\mathcal{H}}(i)=k e(\mathcal{H}) / N
$$

is the average degree of $\mathcal{H}$ and

$$
\sigma_{d}^{2}(\mathcal{H})=\frac{1}{N} \sum_{i=1}^{N}\left(d_{\mathcal{H}}(i)-\bar{d}(\mathcal{H})\right)^{2}
$$

is the variance of the degree sequence of $\mathcal{H}$.

It is natural to state the result as an asymptotic result for a sequence of hypergraphs. We set $t:=m / N$ to be the density of the random set. The result makes sense with $t \in(0,1 / 2]$ a constant or a sequence $t=t_{N} \in$ $(0,1 / 2]$. We observe that the result only applies to hypergraphs with $\Theta\left(N^{2}\right)$ edges possessing certain conditions on the variance of the degree sequence, which correspond to the hypergraph being far from regular. Warnke [20] also considered hypergraphs with $\Theta\left(N^{2}\right)$ edges and some other restrictions. Many natural examples, such as the hypergraphs encoding $k$-APs and Schur triples, are covered in both cases.

Theorem 1.7 Let $\mathcal{H}_{N}$ be a sequence of $k$-uniform hypergraphs $(k \geqslant 2)$ such that $V\left(\mathcal{H}_{N}\right)=[N]$ with maximum degree $\Delta\left(\mathcal{H}_{N}\right)=\Theta(N)$, maximum pair degree $\Delta_{2}\left(\mathcal{H}_{N}\right)=O(1)$ and let $m / N=t \leqslant 1 / 2$. Assume that $\sigma_{d}\left(\mathcal{H}_{N}\right)=\Theta(N)$ and $t \gg(\log N / N)^{1 /(k-1)}$. Let $a_{N}$ be a sequence such that

$$
\max \left\{t N, t^{k-1 / 2} N^{3 / 2}\right\} \ll a_{N} \ll t^{3 k / 2-1} N^{2}
$$

Then

$$
\mathbb{P}\left(D^{\mathcal{H}_{N}}\left(B_{m}\right) \geqslant a_{N}\right)=\exp \left(\frac{-(1+o(1)) a_{N}^{2}}{2(1-t) t^{2 k-1} \sigma_{d}^{2}\left(\mathcal{H}_{N}\right) N}\right) .
$$

This theorem will be a consequence of a slightly more general version, Theorem 6.1, which we will prove in Chapter 6 .

Armed with Theorem 1.7, we can deduce the following theorem in the model $B \sim B_{p}$. We observe that the condition $\Delta\left(\mathcal{H}_{N}\right)=\Theta(N)$ required in Theorem 1.7 is implied by the conditions $e\left(\mathcal{H}_{N}\right)=\Theta\left(N^{2}\right)$ and $\Delta_{2}=O(1)$, since $\Delta$ is at least the average degree of the hypergraph and is at most $N$ times the maximum pair degree. 
Theorem 1.8 Let $\mathcal{H}_{N}$ be a sequence of $k$-uniform hypergraphs $(k \geqslant 2)$ such that $V\left(\mathcal{H}_{N}\right)=[N]$ with $e\left(\mathcal{H}_{N}\right)=\Theta\left(N^{2}\right)$, maximum pair degree $\Delta_{2}=O(1)$ and $\sigma_{d}\left(\mathcal{H}_{N}\right)=\Theta(N)$. Let $p=p_{N}$, bounded away from $1 / 2$, be such that $p \gg(\log N / N)^{1 /(k-1)}$ and let $\delta_{N}$ be a sequence such that

$$
\max \left\{\frac{1}{p^{k-1} N}, \frac{1}{\sqrt{p N}}\right\} \ll \delta_{N} \ll p^{k / 2-1} .
$$

Then

$\mathbb{P}\left(D^{\mathcal{H}_{N}}\left(B_{p}\right) \geqslant \delta_{N} L^{\mathcal{H}_{N}}(p)\right)=\exp \left(\frac{-(1+o(1)) \delta_{N}^{2} p e\left(\mathcal{H}_{N}\right)^{2}}{2(1-p)\left(\bar{d}\left(\mathcal{H}_{N}\right)^{2}+\sigma_{d}^{2}\left(\mathcal{H}_{N}\right)\right) N}+O(\log N)\right)$

Remark 1.9 Replacing the condition $\delta_{N} \gg 1 / \sqrt{p N}$ by

$$
\delta_{N} \gg \sqrt{\frac{\log N}{p N}}
$$

we can drop the $\log N$ term inside the exponential, obtaining

$$
\mathbb{P}\left(D^{\mathcal{H}_{N}}\left(B_{p}\right) \geqslant \delta_{N} L^{\mathcal{H}_{N}}(p)\right)=\exp \left(\frac{-(1+o(1)) \delta_{N}^{2} p e\left(\mathcal{H}_{N}\right)^{2}}{2(1-p)\left(\bar{d}\left(\mathcal{H}_{N}\right)^{2}+\sigma_{d}^{2}\left(\mathcal{H}_{N}\right)\right) N}\right)
$$

This new condition on $\delta_{N}$ also implies, together with the other conditions, that $p \gg(\log N / N)^{1 /(k-1)}$.

As a consequence of the theorem above, consider the hypergraph $\mathcal{H}^{3}$ of increasing $3-\mathrm{APs}$ in $[N]$. It is easy to verify that (indeed it follows from Lemmas 8.6 and 2.1)

$$
\begin{aligned}
\bar{d}\left(\mathcal{H}^{3}\right) & =(1+o(1)) \frac{3 N}{4} \\
\sigma_{d}^{2}\left(\mathcal{H}^{3}\right) & =(1+o(1)) \frac{N^{2}}{48} \\
e\left(\mathcal{H}^{3}\right) & =(1+o(1)) \frac{N^{2}}{4}
\end{aligned}
$$

It is clear that $e\left(\mathcal{H}^{3}\right)=\Theta\left(N^{2}\right), \Delta_{2}\left(\mathcal{H}^{3}\right)=O(1)$ and $\sigma_{d}\left(\mathcal{H}^{3}\right)=\Theta(N)$. Thus Theorem 1.8 and Remark 1.9 give us the following result.

Corollary 1.10 Let $\delta_{N}$ be a sequence satisfying

$$
\max \left\{\frac{1}{p^{2} N}, \sqrt{\frac{\log N}{p N}}\right\} \ll \delta_{N} \ll p^{1 / 2} .
$$

If $p$ is bounded away from $1 / 2$, then 


$$
\mathbb{P}\left(D^{\mathcal{H}^{3}}\left(B_{p}\right) \geqslant \delta_{N} L^{\mathcal{H}_{3}}(p)\right)=\exp \left(\frac{-(3+o(1)) \delta_{N}^{2} p N}{56(1-p)}\right)
$$

Bhattacharya, Ganguly, Shao and Zhao [21] have proved that (1-3) holds under the following conditions on $p$ and $\delta_{N}: p \rightarrow 0, \delta_{N}=O(1)$, $\delta_{N}^{-3} p(\log (1 / p))^{2} \rightarrow \infty$, and

$$
\min \left\{\delta_{N} p^{3}, \delta_{N}^{2} p\right\} \geqslant N^{-1 / 6}(\log N)^{7 / 6}
$$

We observe that Corollary 1.10 extends in some sense their result. Indeed, let us consider once more for simplicity the cases $p=N^{-\gamma}$ and $\delta_{N}=N^{-\theta}$, where $\gamma, \theta>0$.

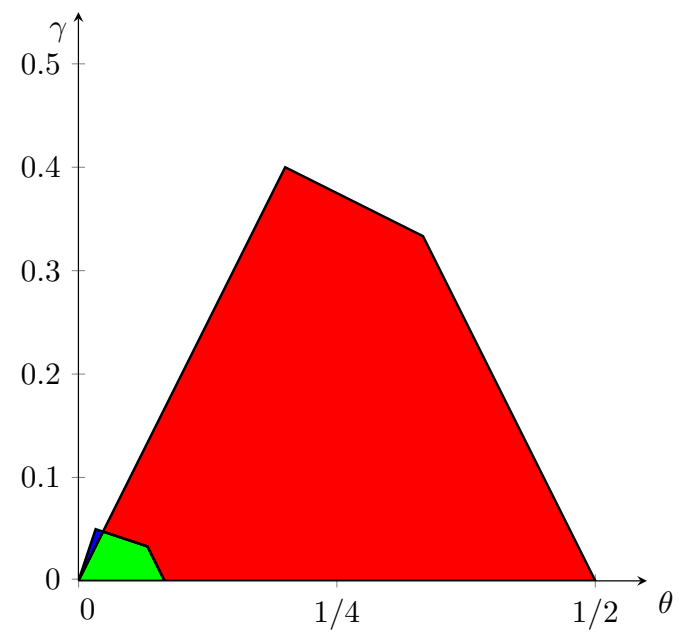

Figure 1.2: The result of Bhattacharya, Ganguly, Shao and Zhao [21] applies in the blue and the green regions. On the other hand, Corollary 1.6 applies in the green and the red regions.

\section{2}

\section{Layout of the thesis}

We now describe the organization of this thesis. In Chapter 2, we introduce some standard definitions regarding sets, asymptotic notations, and hypergraphs. We also recall the definitions of conditional expectation and martingales and we provide some martingale concentration inequalities that will be widely used throughout the thesis. We finish the chapter presenting some estimates for the binomial distribution.

In Chapter 3, we describe the general setup of our problem and we introduce notations that allow us to deduce a martingale representation for 
the deviation $D^{\mathcal{H}}\left(B_{m}\right)$, which may be of independent interest. We also prove a variant of the Hoeffding-Azuma inequality.

In Chapter 4, we obtain deviations for near-regular hypergraphs in the $m$-model (Theorem 1.2). This is achieved by a double induction argument which links deviation probabilities to the increments of the martingale and vice-versa.

In Chapter 5, we obtain deviations for near-regular hypergraphs in the $p$-model (Theorem 1.5) by transferring in a quite standard way the result from Theorem 1.2.

In Chapter 6, we prove Theorem 1.7 about deviations in non regular hypergraphs in the $m$-model as a corollary of Theorem 6.1 . The idea is to prove that $D^{\mathcal{H}}\left(B_{m}\right)$ is very well approximated by an expression which only depends on the degrees of the vertices of $B_{m}$. After that we prove a result in the same spirit of Theorem 6.1 for this expression, which is easier to analyze.

In Chapter 7 , we again transfer the results from the $m$-model to the $p$-model, proving then Theorem 1.8 .

Finally, in Chapter 8, we collect some applications of Theorems 1.2, 1.5, 1.7, and 1.8 for a variety of arithmetic structures. These include $k$-arithmetic progressions, Schur triples, additive quadruples, and, more generally, solutions of linear systems in random sets obtained from $\mathbb{Z}_{N}$, with $N$ prime. We also provide results for $k$-arithmetic progressions and Schur triples in random sets obtained from $[N]$. 


\section{2}

\section{Preliminaries}

In this chapter we recall some definitions and results that will be used throughout the thesis.

\section{1}

\section{Basic notations}

We denote the set of natural numbers by $\mathbb{N}=\{0,1,2, \ldots\}$. For a positive integer $n$, we denote by $[n]$ the set of the first $n$ positive integers, i.e., $[n]:=\{1, \ldots, n\}$ and $\mathbb{Z}_{n}$ denotes the additive cyclic group of integers modulo $n$. Given a finite set $A,|A|$ is the cardinality of $A$ and for a positive integer $k,\left(\begin{array}{l}A \\ k\end{array}\right)$ is the set whose elements are all the $k$-element subsets of $A$. If $|A|=n$, we observe that

$$
\left|\left(\begin{array}{l}
A \\
k
\end{array}\right)\right|=\left(\begin{array}{l}
n \\
k
\end{array}\right)=\frac{n !}{k !(n-k) !} .
$$

Given a real number $x$ and a positive integer $k$, the falling factorial $(x)_{k}$ is defined as the polynomial

$$
(x)_{k}=\prod_{j=1}^{k}(x-j+1) .
$$

We also define $(x)_{0}=1$ for all real numbers $x$.

\section{2}

\section{Asymptotic notations}

In this section, $\left(a_{n}\right)$ and $\left(b_{n}\right)$ denote sequences of real numbers, where $\left(b_{n}\right)$ is positive for all sufficiently large $n$. We now introduce the asymptotic notations that are widely used in this work. We write $a_{n}=O\left(b_{n}\right)$ if there exists a constant $C>0$ such that

$$
\left|a_{n}\right| \leqslant C b_{n}
$$

for all sufficiently large $n$. In a similar way, we write $a_{n}=\Omega\left(b_{n}\right)$ if there exists a constant $c>0$ such that

$$
a_{n} \geqslant c b_{n}
$$


for all sufficiently large $n$.

When $a_{n}=O\left(b_{n}\right)$ and $a_{n}=\Omega\left(b_{n}\right)$, we write $a_{n}=\Theta\left(b_{n}\right)$, i.e., there are constants $c, C>0$ such that

$$
c b_{n} \leqslant a_{n} \leqslant C b_{n}
$$

for all sufficiently large $n$.

Sometimes the constants may depend on other quantities. In such cases, we denote for example $a_{n}=O_{x}\left(b_{n}\right)$ (and similarly for $\Omega$ and $\Theta$ ) if there exists a constant $C(x)>0$, depending on $x$, such that

$$
\left|a_{n}\right| \leqslant C(x) b_{n}
$$

for all sufficiently large $n$. We write $a_{n}=o\left(b_{n}\right)$ or $a_{n} \ll b_{n}$ if

$$
\lim _{n \rightarrow \infty} \frac{a_{n}}{b_{n}}=0
$$

On the other hand, we write $a_{n}=\omega\left(b_{n}\right)$ or $a_{n} \gg b_{n}$ if

$$
\lim _{n \rightarrow \infty} \frac{a_{n}}{b_{n}}=\infty
$$

\section{3}

\section{Hypergraphs}

In this section, we introduce the basic notation regarding hypergraphs. A hypergraph $\mathcal{H}$ is a pair $\mathcal{H}=(V(\mathcal{H}), E(\mathcal{H}))$ of sets, where the elements of $E(\mathcal{H})$ (set of edges) are non-empty subsets of $V(\mathcal{H})$ (set of vertices). We usually denote by $e(\mathcal{H})$ the number of edges of $\mathcal{H}$, i.e., $e(\mathcal{H}):=|E(\mathcal{H})|$. For a positive integer $k$, we say that a hypergraph $\mathcal{H}$ is $k$-uniform if $E(\mathcal{H}) \subset\left(\begin{array}{c}V(\mathcal{H}) \\ k\end{array}\right)$, i.e., if all edges of $\mathcal{H}$ have size $k$.

Given a hypergraph $\mathcal{H}=(V(\mathcal{H}), E(\mathcal{H}))$ and a subset $A \subset V(\mathcal{H})$, we define the degree of $A$ in $\mathcal{H}$, denoted by $d_{\mathcal{H}}(A)$, as the number of edges of $\mathcal{H}$ containing $A$. For a positive integer $\ell$, the maximum $\ell$-degree of $\mathcal{H}$, denoted by $\Delta_{\ell}(\mathcal{H})$, is the maximum degree of $A$ over all $A \in\left(\begin{array}{c}V(\mathcal{H}) \\ \ell\end{array}\right)$. When $\ell=1$, we usually write $\Delta_{1}(\mathcal{H})=\Delta(\mathcal{H})$.

We also define the average $\ell$-degree of $\mathcal{H}$, denoted by $d_{\ell}(\mathcal{H})$ by

$$
\bar{d}_{\ell}(\mathcal{H})=\frac{\sum_{A \subset\left(\begin{array}{c}
V(\mathcal{H}) \\
\ell
\end{array}\right)} d_{\mathcal{H}}(A)}{\left(\begin{array}{c}
|V(\mathcal{H})| \\
\ell
\end{array}\right)} .
$$

When $\ell=1$, we usually write $\bar{d}_{1}(\mathcal{H})=\bar{d}(\mathcal{H})$. 
We now state the following simple result, which is a generalization of the classical handshaking lemma for graphs, and that can be proved by double counting the number of pairs $(A, e)$ with $A \subset e$, where $A$ is an $\ell$-subset of $V(\mathcal{H})$ and $e$ is an edge of the hypergraph.

Lemma 2.1 Let $\mathcal{H}=(V(\mathcal{H}), E(\mathcal{H}))$ be a $k$-uniform hypergraph and $\ell$ be a positive integer. Then

$$
\bar{d}_{\ell}(\mathcal{H})=\frac{e(\mathcal{H})\left(\begin{array}{l}
k \\
\ell
\end{array}\right)}{\left(\begin{array}{c}
|V(\mathcal{H})| \\
\ell
\end{array}\right)}
$$

Some hypergraphs have the nice property that all $r$-tuples of vertices are included in the same number of edges. We make this precise in the following definition.

Definition 2.2 We say that a hypergraph $\mathcal{H}=(V(\mathcal{H}), E(\mathcal{H}))$ is r-tupleregular if for each pair $A, A^{\prime} \in\left(\begin{array}{c}V(\mathcal{H}) \\ r\end{array}\right)$ we have $d_{\mathcal{H}}(A)=d_{\mathcal{H}}\left(A^{\prime}\right)$.

Unfortunately some natural hypergraphs are not precisely $r$-tupleregular, but just very close to being so.

Definition 2.3 We say that a hypergraph $\mathcal{H}=(V(\mathcal{H}), E(\mathcal{H}))$ is $(r, \eta)$-nearregular if for each $A \in\left(\begin{array}{c}V(\mathcal{H}) \\ r\end{array}\right)$, we have $d_{\mathcal{H}}(A)=(1 \pm \eta) \bar{d}_{r}(\mathcal{H})$.

Furthermore, we denote by $\mathcal{H}(x)$ the link hypergraph with respect to the vertex $x \in V(\mathcal{H})$, that is the hypergraph with vertex set $V(\mathcal{H}(x))=V(\mathcal{H}) \backslash\{x\}$ and edge set

$$
E(\mathcal{H}(x)):=\{f \backslash\{x\}: f \in E(\mathcal{H}), x \in f\}
$$

In the case that $\mathcal{H}$ is a $k$-uniform hypergraph on $[N]:=\{1, \ldots, N\}$, then $\mathcal{H}(x)$ is a $(k-1)$-uniform hypergraph on $[N] \backslash\{x\}$. The following lemma indicates some ways in which near-regularity is inherited.

Lemma 2.4 Let $\mathcal{H}$ be a $k$-uniform $(r, \eta)$-near-regular hypergraph $(1 \leqslant r \leqslant k)$ with maximum $(r+1)$-degree $\Delta_{r+1}$, for some $\eta \in[0,1 / 3]$. Let $x \in V(\mathcal{H})$. Then

(i) $\mathcal{H}$ is $(r-1, \eta)$-near-regular.

(ii) $\mathcal{H}(x)$ is $(r-1,3 \eta)$-near-regular with maximum $r$-degree at most $\Delta_{r+1}$.

Proof. Part (i) follows from a double counting argument. Indeed, let us fix $A \subset\left(\begin{array}{l}{[N]} \\ r-1\end{array}\right)$ and consider the triples $(A, B, e)$ such that $A \subset B \subset e$, where $B \subset\left(\begin{array}{c}{[N]} \\ r\end{array}\right)$ and $e$ is an edge of the hypergraph. First we observe that the number of such triples is

$$
\sum_{x \notin A} d_{\mathcal{H}}(A \cup\{x\}) .
$$


Since $\mathcal{H}$ is $(r, \eta)$-near-regular, each term in the above sum is between $(1-\eta) \bar{d}_{r}(\mathcal{H})$ and $(1+\eta) \bar{d}_{r}(\mathcal{H})$. As there are $N-r+1$ terms in the sum, the number of triples is

$$
(1 \pm \eta)(N-r+1) \bar{d}_{r}(\mathcal{H})
$$

On the other hand, if we start by counting from $e$, the number of triples is

$$
(k-r+1) d_{\mathcal{H}}(A) .
$$

Altogether we obtain

$$
(1-\eta) \frac{N-r+1}{k-r+1} \bar{d}_{r}(\mathcal{H}) \leqslant d_{\mathcal{H}}(A) \leqslant(1+\eta) \frac{N-r+1}{k-r+1} \bar{d}_{r}(\mathcal{H}) .
$$

Finally, Lemma 2.1 gives us that

$$
\bar{d}_{r-1}(\mathcal{H})=\frac{N-r+1}{k-r+1} \bar{d}_{r}(\mathcal{H})
$$

which proves that $\mathcal{H}$ is $(r-1, \eta)$-near-regular.

For (ii) it is easy to observe that $\mathcal{H}(x)$ is $(k-1)$-uniform with maximum $r$-degree at most $\Delta_{r+1}$. We will prove now that $\mathcal{H}(x)$ is $(r-1,3 \eta)$-near-regular. Since $\mathcal{H}$ is $(r, \eta)$-near-regular, it is also $(1, \eta)$-near-regular, by (i), and therefore

$$
d_{\mathcal{H}}(x) \geqslant(1-\eta) \bar{d}_{1}(\mathcal{H})=(1-\eta) \frac{e(\mathcal{H}) k}{N}
$$

by Lemma 2.1, where $N=|V(\mathcal{H})|$. And so it follows again from Lemma 2.1 that the average $(r-1)$-degree of $\mathcal{H}(x)$ satisfies

$$
\bar{d}_{r-1}(\mathcal{H}(x))=\frac{e(\mathcal{H}(x))\left(\begin{array}{l}
k-1 \\
r-1
\end{array}\right)}{\left(\begin{array}{c}
N-1 \\
r-1
\end{array}\right)}=\frac{(k-1)_{r-1}}{(N-1)_{r-1}} d_{\mathcal{H}}(x),
$$

since $e(\mathcal{H}(x))=d_{\mathcal{H}}(x)$. Once again from Lemma 2.1, we have

$$
\frac{(k-1)_{r-1}}{(N-1)_{r-1}}=\bar{d}_{r}(\mathcal{H}) \frac{N}{e(\mathcal{H}) k} .
$$

Therefore we obtain by (2-1), (2-2) and (2-3) that

$$
\bar{d}_{r-1}(\mathcal{H}(x))=\bar{d}_{r}(\mathcal{H}) \cdot \frac{N}{e(\mathcal{H}) k} \cdot d_{\mathcal{H}}(x) \geqslant(1-\eta) \bar{d}_{r}(\mathcal{H})
$$

Now let $A \subseteq V(\mathcal{H}(x))$ be an $(r-1)$-element set. Since $\mathcal{H}$ is $(r, \eta)$-nearregular we have

$$
d_{\mathcal{H}(x)}(A)=d_{\mathcal{H}}(A \cup\{x\}) \leqslant(1+\eta) \bar{d}_{r}(\mathcal{H})
$$


and so, since $\eta \in[0,1 / 3]$, we have

$$
d_{\mathcal{H}(x)}(A) \leqslant \frac{1+\eta}{1-\eta} \bar{d}_{r-1}(\mathcal{H}(x)) \leqslant(1+3 \eta) \bar{d}_{r-1}(\mathcal{H}(x)) .
$$

A near identical argument gives the lower bound $(1-\eta) /(1+\eta) \geqslant 1-3 \eta$ times $\bar{d}_{r-1}(\mathcal{H}(x))$, and so completes the proof.

Another quantity of interest for us is the variance of the degree sequence of a hypergraph. We have already defined this in the introduction, but now we restate the definition in another useful way.

Definition 2.5 Given a hypergraph $\mathcal{H}=(V(\mathcal{H}), E(\mathcal{H}))$, the variance of its degree sequence is defined by

$$
\sigma_{d}^{2}(\mathcal{H})=\frac{\sum_{x \in V(\mathcal{H})} d_{\mathcal{H}}(x)^{2}}{|V(\mathcal{H})|}-\left(\frac{\sum_{x \in V(\mathcal{H})} d_{\mathcal{H}}(x)}{|V(\mathcal{H})|}\right)^{2}
$$

\section{4}

\section{Conditional expectation and Martingales}

We first recall the definition of conditional expectation.

Definition 2.6 Let $(\Omega, \mathbb{F}, \mathbb{P})$ be a probability space and $X: \Omega \rightarrow \mathbb{R}$ a random variable with $\mathbb{E}[|X|]<\infty$ (we say that $X \in L^{1}(\Omega)$ ). Let $\mathcal{G}$ be a sub- $\sigma$-algebra of $\mathbb{F}$. A conditional expectation of $X$ given $\mathcal{G}$, denoted by $\mathbb{E}[X \mid \mathcal{G}]$, is any $\mathcal{G}$ measurable random variable $Y \in L^{1}(\Omega)$ such that

$$
\int_{G} Y d \mathbb{P}=\int_{G} X d \mathbb{P}
$$

for every $G \in \mathcal{G}$.

The following theorem, whose proof can be found in [23], establishes the existence and the almost sure uniqueness of $\mathbb{E}[X \mid \mathcal{G}]$.

Theorem 2.7 Let $(\Omega, \mathbb{F}, \mathbb{P})$ be a probability space and $X \in L^{1}(\Omega)$. Let $\mathcal{G}$ be a sub- $\sigma$-algebra of $\mathbb{F}$. Then there exists a $\mathcal{G}$-measurable random variable $Y \in L^{1}(\Omega)$ such that

$$
\int_{G} Y d \mathbb{P}=\int_{G} X d \mathbb{P}
$$

for every $G \in \mathcal{G}$. Moreover, if $\tilde{Y}$ is another random variable with these properties, then $\tilde{Y}=Y$ a.s.

Remark 2.8 We often write $\mathbb{E}\left[X \mid Z_{1}, \ldots, Z_{k}\right]$ for $\mathbb{E}\left[X \mid \sigma\left(Z_{1}, \ldots, Z_{k}\right)\right]$. 
We now present some useful properties about conditional expectation, whose proofs can also be found in [23].

Lemma 2.9 Let $(\Omega, \mathbb{F}, \mathbb{P})$ be a probability space and $\mathcal{G}$ a sub- $\sigma$-algebra of $\mathbb{F}$. The following properties hold.

(i) If $X \in L^{1}(\Omega)$, then $\mathbb{E}[\mathbb{E}[X \mid \mathcal{G}]]=\mathbb{E}[X]$.

(ii) If $X, Y \in L^{1}(\Omega)$ and $\alpha \in \mathbb{R}$, then $\mathbb{E}[\alpha X+Y \mid \mathcal{G}]=\alpha \mathbb{E}[X \mid \mathcal{G}]+\mathbb{E}[Y \mid \mathcal{G}]$ a.s.

(iii) If $X, Y \in L^{1}(\Omega)$ and $X \geqslant Y$ a.s., then $\mathbb{E}[X \mid \mathcal{G}] \geqslant \mathbb{E}[Y \mid \mathcal{G}]$ a.s.

(iv) If $Z$ is a $\mathcal{G}$-measurable random variable bounded a.s. and $X \in L^{1}(\Omega)$, then $\mathbb{E}[Z X \mid \mathcal{G}]=Z \mathbb{E}[X \mid \mathcal{G}]$.

Finally we introduce the concept of martingales. Given a probability space $(\Omega, \mathcal{F}, \mathbb{P})$, we say that an increasing family of sub- $\sigma$-algebras $\left(\mathcal{F}_{n}\right)_{n \geqslant 0}$ is a filtration.

A sequence of random variables $\left(S_{n}\right)_{n \geqslant 0}$ is called a martingale with respect to a filtration $\left(\mathcal{F}_{n}\right)_{n \geqslant 0}$ if for all $n \geqslant 0$

(i) $S_{n}$ is $\mathcal{F}_{n}$-measurable,

(ii) $S_{n} \in L^{1}(\Omega)$,

(iii) $\mathbb{E}\left[S_{n+1} \mid \mathcal{F}_{n}\right]=S_{n}$ a.s.

Remark 2.10 We say that $\left(S_{n}\right)_{n \geqslant 0}$ is simply a martingale when the filtration is $\mathcal{F}_{n}=\sigma\left(S_{0}, S_{1}, \ldots, S_{n}\right)$. 


\section{5}

\section{Martingale concentration inequalities}

In this section we recall some martingale inequalities that will play a key role in this thesis. We first observe that if $X$ is a non-negative random variable and $a>0$, then $X \geqslant a \cdot 1_{\{X \geqslant a\}}$, where $1_{A}$ is the indicator function of the event $A$. By taking expectation on both sides, we obtain Markov's inequality.

Lemma 2.11 (Markov inequality) Let $X$ be a non-negative random variable and $a>0$. Then

$$
\mathbb{P}(X \geqslant a) \leqslant \frac{\mathbb{E}[X]}{a} .
$$

We now prove the Hoeffding-Azuma inequality [24, 25], which applies for martingales with bounded increments.

Lemma 2.12 (Hoeffding-Azuma inequality) Let $\left(S_{m}\right)_{m=0}^{M}$ be a martingale such that $\left|S_{i}-S_{i-1}\right| \leqslant c_{i}$ a.s. for each $1 \leqslant i \leqslant M$. Then, for every $a>0$,

$$
\mathbb{P}\left(S_{M}-S_{0}>a\right) \leqslant \exp \left(\frac{-a^{2}}{2 \sum_{i=1}^{M} c_{i}^{2}}\right) .
$$

Furthermore, the same bound holds for $\mathbb{P}\left(S_{M}-S_{0}<-a\right)$.

In order to prove this inequality we need two auxiliary lemmas.

Lemma 2.13 For every real number $x$, it holds that

$$
\frac{\exp (x)+\exp (-x)}{2}:=\cosh x \leqslant \exp \left(\frac{x^{2}}{2}\right) .
$$

Proof. By Taylor series expansion, we have

$$
\cosh x=\sum_{n=0}^{\infty} \frac{x^{2 n}}{(2 n) !} \leqslant \sum_{n=0}^{\infty} \frac{x^{2 n}}{2^{n} \cdot n !}=\exp \left(\frac{x^{2}}{2}\right),
$$

where we used that $(2 n) ! \geqslant 2^{n} \cdot n !$ for every integer $n \geqslant 0$.

Lemma 2.14 Let $Y$ be a random variable such that $|Y| \leqslant c$ a.s. and $\mathbb{E}[Y \mid \mathcal{F}]=0$ a.s. Then, for every $\lambda>0$,

$$
\mathbb{E}[\exp (\lambda Y) \mid \mathcal{F}] \leqslant \exp \left(\frac{\lambda^{2} c^{2}}{2}\right) \text { a.s. }
$$

Proof. For every $y \in[-c, c]$, by convexity we have

$$
\exp (\lambda y) \leqslant \frac{\exp (-\lambda c)+\exp (\lambda c)}{2}+\frac{y}{c} \cdot \frac{\exp (\lambda c)-\exp (-\lambda c)}{2} .
$$


Since $|Y| \leqslant c$ a.s., it follows that

$$
\mathbb{E}[\exp (\lambda Y) \mid \mathcal{F}] \leqslant \mathbb{E}\left[\frac{\exp (-\lambda c)+\exp (\lambda c)}{2}+\frac{Y}{c} \cdot \frac{\exp (\lambda c)-\exp (-\lambda c)}{2} \mid \mathcal{F}\right]
$$

As $\mathbb{E}[Y \mid \mathcal{F}]=0$ a.s., Lemma 2.13 gives us

$$
\mathbb{E}\left[e^{\lambda Y} \mid \mathcal{F}\right] \leqslant \exp \left(\frac{\lambda^{2} c^{2}}{2}\right) \text { a.s. }
$$

We are now in position to prove the Hoeffding-Azuma inequality.

Proof of Lemma 2.12. For $1 \leqslant i \leqslant M$, let $X_{i}=S_{i}-S_{i-1}$ and observe that $S_{M}-S_{0}=\sum_{i=1}^{M} X_{i}$ and $\mathbb{E}\left[X_{i} \mid \mathcal{F}_{i-1}\right]=0$. By properties (i) and (iv) of Lemma 2.9 , we have

$$
\begin{aligned}
\mathbb{E}\left[\exp \left(\lambda\left(S_{M}-S_{0}\right)\right]\right. & =\mathbb{E}\left[\exp \left(\lambda \sum_{i=1}^{M} X_{i}\right)\right] \\
& =\mathbb{E}\left[\exp \left(\lambda X_{M}\right) \cdot \exp \left(\lambda \sum_{i=1}^{M-1} X_{i}\right)\right] \\
& =\mathbb{E}\left[\mathbb{E}\left[\exp \left(\lambda X_{M}\right) \cdot \exp \left(\lambda \sum_{i=1}^{M-1} X_{i}\right) \mid \mathcal{F}_{M-1}\right]\right] \\
& =\mathbb{E}\left[\exp \left(\lambda \sum_{i=1}^{M-1} X_{i}\right) \cdot \mathbb{E}\left[\exp \left(\lambda X_{M}\right) \mid \mathcal{F}_{M-1}\right]\right]
\end{aligned}
$$

Applying Lemma 2.14 with the random variable $X_{M}$, we obtain

$$
\mathbb{E}\left[\exp \left(\lambda\left(S_{M}-S_{0}\right)\right] \leqslant \exp \left(\frac{\lambda^{2} c_{M}^{2}}{2}\right) \cdot \mathbb{E}\left[\exp \left(\lambda \sum_{i=1}^{M-1} X_{i}\right)\right] .\right.
$$

Therefore, by induction it follows that

$$
\mathbb{E}\left[\exp \left(\lambda\left(S_{M}-X_{0}\right)\right] \leqslant \exp \left(\frac{\lambda^{2}}{2} \sum_{i=1}^{M} c_{i}^{2}\right)\right.
$$

For $\lambda>0$, Markov's inequality gives us

$$
\begin{aligned}
\mathbb{P}\left(S_{M}-S_{0}>a\right) & =\mathbb{P}\left(\exp \left(\lambda\left(S_{M}-S_{0}\right)\right)>\exp (\lambda a)\right) \\
& \leqslant \frac{\mathbb{E}\left[\exp \left(\lambda\left(S_{M}-S_{0}\right)\right]\right.}{\exp (\lambda a)} \\
& \leqslant \exp \left(\frac{\lambda^{2}}{2} \sum_{i=1}^{M} c_{i}^{2}-\lambda a\right) .
\end{aligned}
$$


We now finish the proof choosing $\lambda=a /\left(\sum_{i=1}^{M} c_{i}^{2}\right)$. The furthermore part follows replacing $S_{m}$ by $-S_{m}$, which still is a martingale.

Sometimes the martingale increments are typically much smaller than their maximum possible value. One alternative to Hoeffding-Azuma inequality in such cases is Freedman's inequality [26], which takes into account the conditional second moment of the increments, $\mathbb{E}\left[X_{i}^{2} \mid \mathcal{F}_{i-1}\right]$.

Lemma 2.15 (Freedman's inequality) Let $\left(S_{m}\right)_{m=0}^{M}$ be a martingale with increments $\left(X_{i}\right)_{i=1}^{M}$ with respect to a filtration $\left(\mathcal{F}_{m}\right)_{m=0}^{M}$, let $R \in \mathbb{R}$ be such that $\max _{i}\left|X_{i}\right| \leqslant R$ almost surely, and let

$$
V(m):=\sum_{i=1}^{m} \mathbb{E}\left[X_{i}^{2} \mid \mathcal{F}_{i-1}\right]
$$

Then, for every $\alpha, \beta>0$, we have

$$
\mathbb{P}\left(S_{m}-S_{0} \geqslant \alpha \text { and } V(m) \leqslant \beta \text { for some } m\right) \leqslant \exp \left(\frac{-\alpha^{2}}{2(\beta+R \alpha)}\right) .
$$

Freedman also proved a converse for this inequality, which requires some new notations. Define the stopping time $m_{\alpha}$ to be the least $m$ such that $S_{m}>S_{0}+\alpha$ (we let $m_{\alpha}=\infty$ if there is no such $m$ ) and

$$
T_{\alpha}:=V\left(m_{\alpha}\right)
$$

We observe that $T_{\alpha}$ is the total amount of conditional variance it takes for the martingale process to cross the $\alpha$-line, if it crosses it; otherwise $T_{\alpha}$ is the total amount of conditional variance. Freedman's converse inequality [26] is as follows.

Lemma 2.16 (Converse Freedman inequality) Let $\left(S_{m}\right)_{i=0}^{M}$ be a martingale with increments $\left(X_{i}\right)_{i=1}^{M}$ with respect to a filtration $\left(\mathcal{F}_{m}\right)_{m=0}^{M}$, let $R \in \mathbb{R}$ be such that $\max _{i}\left|X_{i}\right| \leqslant R$ almost surely, and let $T_{\alpha}$ be defined as above. Then for every $\alpha, \beta>0$, we have

$$
\mathbb{P}\left(T_{\alpha} \leqslant \beta\right) \geqslant \frac{1}{2} \exp \left(\frac{-\alpha^{2}(1+4 \delta)}{2 \beta}\right),
$$

where $\delta$ is minimal such that $\beta / \alpha \geqslant 9 R \delta^{-2}$ and $\alpha^{2} / \beta \geqslant 16 \delta^{-2} \log \left(64 \delta^{-2}\right)$. 


\section{6}

\section{Estimates for the binomial distribution}

When transferring the results from the $m$-model to the $p$-model, we will make use of some estimates for tail probabilities of the binomial distribution. Let us denote by $\operatorname{Bin}(N, p)$ the binomial distribution with parameters $N$ and $p$ and set $q:=1-p$. We also define

$$
b_{N, p}(m):=\mathbb{P}(\operatorname{Bin}(N, p)=m)
$$

and

$$
B_{N, p}(m):=\mathbb{P}(\operatorname{Bin}(N, p) \geqslant m) .
$$

For $0<x<\sqrt{N} / 2$, define

$$
E(x, N)=\sum_{i=1}^{\infty} \frac{\left(p^{i+1}+(-1)^{i} q^{i+1}\right) x^{i+2}}{(i+1)(i+2) p^{i / 2} q^{i / 2} N^{i / 2}} .
$$

The result we give next is essentially due to Bahadur [27], adapted in the appendix of [18].

Theorem 2.17 Suppose that $\left(x_{N}\right)$ is a sequence such that $1 \ll x_{N} \ll \sqrt{N p q}$. Then

$$
b_{N, p}\left(\left\lfloor p N+x_{N} \sqrt{N p q}\right\rfloor\right)=(1+o(1)) \frac{1}{\sqrt{2 \pi N p q}} \exp \left(-\frac{x_{N}^{2}}{2}-E\left(x_{N}, N\right)\right)
$$

and

$$
B_{N, p}\left(\left\lfloor p N+x_{N} \sqrt{N p q}\right\rfloor\right)=(1+o(1)) \frac{1}{x_{N} \sqrt{2 \pi}} \exp \left(-\frac{x_{N}^{2}}{2}-E\left(x_{N}, N\right)\right) .
$$




\section{3}

\section{General setup of the problem}

We aim to study deviation probabilities for the number of edges in random subhypergraphs. Given $N \in \mathbb{N}$, let $\mathcal{H}=(V(\mathcal{H}), E(\mathcal{H}))$ be a hypergraph on the vertex set $V(\mathcal{H}):=[N]$, where $[N]=\{1,2, \ldots, N\}$. For an integer $m \in \mathbb{N}$, let $B_{m} \subseteq[N]$ denote a uniformly randomly chosen subset of size $\left|B_{m}\right|=m$. We observe that we can consider $B_{m}$ as being obtained by an $m$-step process such that in each step we choose an element for $B_{m}$ uniformly among all the non previously chosen elements of $[N]$. Throughout the thesis, we consider the subhypergraph $\mathcal{H}\left[B_{m}\right]$ induced by the set $B_{m}$, i.e., its vertex set is $B_{m}$ and it contains all edges of $\mathcal{H}$ which are completely contained in $B_{m}$.

The heart of our method is based on a martingale expression for the deviation of the random variable $e\left(\mathcal{H}\left[B_{m}\right]\right)$. In other words, we consider the random variable $D^{\mathcal{H}}\left(B_{m}\right):=e\left(\mathcal{H}\left[B_{m}\right]\right)-\mathbb{E}\left[e\left(\mathcal{H}\left[B_{m}\right]\right)\right]$. Thus in the next section we will introduce notations that will allow us to deduce a martingale representation for $D^{\mathcal{H}}\left(B_{m}\right)$.

\section{1}

\section{Notations}

First of all, we consider $B_{m}$ as an ordered set $B_{m}=\left\{b_{1}, b_{2}, \ldots, b_{m}\right\}$ and for $i \leqslant m$, we denote $B_{i}=\left\{b_{1}, b_{2}, \ldots, b_{i}\right\}$. For convenience, we will write $t:=m / N$ for the density of the random set $B_{m} \subseteq[N]$; similarly, when considering earlier steps of the process, say $B_{i}$ with $1 \leqslant i \leqslant m$, we abbreviate the density by $s:=i / N$. Next, we let $N^{\mathcal{H}}\left(B_{m}\right):=e\left(\mathcal{H}\left[B_{m}\right]\right)$ denote the number of edges of $\mathcal{H}$ completely contained in $B_{m}$. However, we will also need to keep track of the numbers of partially completed edges, which we count with multiplicities. Formally, for each $j \in \mathbb{N}$, the number of $j$-subsets of edges contained in $B_{m}$ is denoted by

$$
N_{j}^{\mathcal{H}}\left(B_{m}\right):=\sum_{f \in E(\mathcal{H})}\left(\begin{array}{c}
\left|f \cap B_{m}\right| \\
j
\end{array}\right),
$$

where the summation accounts for counting with multiplicities. Moreover, we write

$$
D_{j}^{\mathcal{H}}\left(B_{m}\right):=N_{j}^{\mathcal{H}}\left(B_{m}\right)-\mathbb{E}\left[N_{j}^{\mathcal{H}}\left(B_{m}\right)\right]
$$


for the deviation of the count of $j$-subsets from its mean. Note that when the hypergraph $\mathcal{H}$ is $k$-uniform, for some $k \in \mathbb{N}$, we have $N^{\mathcal{H}}\left(B_{m}\right)=N_{k}^{\mathcal{H}}\left(B_{m}\right)$ and $D^{\mathcal{H}}\left(B_{m}\right)=D_{k}^{\mathcal{H}}\left(B_{m}\right)$. In this work, we will focus on such hypergraphs.

Throughout the thesis we denote the expectation of $N_{j}^{\mathcal{H}}\left(B_{m}\right)$ by

$$
L_{j}^{\mathcal{H}}(m):=\mathbb{E}\left[N_{j}^{\mathcal{H}}\left(B_{m}\right)\right]=e(\mathcal{H})\left(\begin{array}{l}
k \\
j
\end{array}\right) \frac{(m)_{j}}{(N)_{j}} .
$$

In order to define the increments of the key martingale representation, ${ }^{1}$ we introduce

$$
X_{\ell}^{\mathcal{H}}\left(B_{i}\right):=N_{\ell}^{\mathcal{H}}\left(B_{i}\right)-\mathbb{E}\left[N_{\ell}^{\mathcal{H}}\left(B_{i}\right) \mid B_{i-1}\right] .
$$

Since $N_{\ell}^{\mathcal{H}}\left(B_{i-1}\right)$ is determined by $B_{i-1}$ we observe that

$$
X_{\ell}^{\mathcal{H}}\left(B_{i}\right)=A_{\ell}^{\mathcal{H}}\left(B_{i}\right)-\mathbb{E}\left[A_{\ell}^{\mathcal{H}}\left(B_{i}\right) \mid B_{i-1}\right]
$$

where

$$
A_{\ell}^{\mathcal{H}}\left(B_{i}\right):=N_{\ell}^{\mathcal{H}}\left(B_{i}\right)-N_{\ell}^{\mathcal{H}}\left(B_{i-1}\right)
$$

denotes the increase in $N_{\ell}^{\mathcal{H}}\left(B_{i}\right)$ with the addition of the $i$-th element. Both of the above expressions for $X_{\ell}^{\mathcal{H}}\left(B_{i}\right)$ will be used during the thesis. We observe that, by its definition, the sequence $X_{\ell}^{\mathcal{H}}\left(B_{i}\right)$ is a martingale difference for each $\ell$.

Besides the $m$-model, where we consider uniformly random chosen subsets of cardinality $m$, we will also consider the $p$-model. Here we consider a random subset $B_{p} \subseteq[N]$, in which each element is included in $B_{p}$ independently with probability $p$. As usual in probabilistic combinatorics we allow for the possibility that $p=p(N)$ is a function of $N$. In this model, we denote by $N^{\mathcal{H}}\left(B_{p}\right)$ the number of edges of $\mathcal{H}$ that are contained in $B_{p}$ and we denote the deviation of this random variable from its mean by

$$
D^{\mathcal{H}}\left(B_{p}\right)=N^{\mathcal{H}}\left(B_{p}\right)-L^{\mathcal{H}}(p)
$$

where $L^{\mathcal{H}}(p):=\mathbb{E}\left[N^{\mathcal{H}}\left(B_{p}\right)\right]=e(\mathcal{H}) p^{k}$.

\section{2}

\section{Martingale representation}

We are now in position to state and to prove the following martingale representation for $D_{j}^{\mathcal{H}}\left(B_{m}\right)$, which is one of the key ingredients to our approach.

\footnotetext{
${ }^{1} \mathrm{With}$ respect to the order of the random set $B_{m}$.
} 
Lemma 3.1 Let $\mathcal{H}$ be a $k$-uniform hypergraph and let $0 \leqslant j \leqslant k$. Then

$$
D_{j}^{\mathcal{H}}\left(B_{m}\right)=\sum_{i=1}^{m} \sum_{\ell=1}^{j} \frac{(N-m)_{\ell}(m-i)_{j-\ell}}{(N-i)_{j}}\left(\begin{array}{l}
k-\ell \\
k-j
\end{array}\right) X_{\ell}^{\mathcal{H}}\left(B_{i}\right) .
$$

Proof. We prove the required expression (3-2) by a double induction over $m$ and $j$. The base cases $j=0$ and $m=0$ are trivial.

For the induction step we may assume that (3-2) holds if $j^{\prime}<j$ or if $j^{\prime}=j$ and $m^{\prime}<m$. The argument proceeds by focusing on the latest point added. We recall that $X_{j}^{\mathcal{H}}\left(B_{m}\right)=A_{j}^{\mathcal{H}}\left(B_{m}\right)-\mathbb{E}\left[A_{j}^{\mathcal{H}}\left(B_{m}\right) \mid B_{m-1}\right]$ and that $A_{j}^{\mathcal{H}}\left(B_{m}\right)=N_{j}^{\mathcal{H}}\left(B_{m}\right)-N_{j}^{\mathcal{H}}\left(B_{m-1}\right)$ counts the increase in $N_{j}^{\mathcal{H}}\left(B_{m}\right)$ with the addition of the $m$-th element of $B_{m}$. Considering that any such increase must consist of a $(j-1)$-subset of an edge together with an extra element of the same edge (which is not already present) and each vertex has probability $1 /(N-m+1)$ to be selected next, we have that

$$
\mathbb{E}\left[A_{j}^{\mathcal{H}}\left(B_{m}\right) \mid B_{m-1}\right]=\frac{(k-j+1) N_{j-1}^{\mathcal{H}}\left(B_{m-1}\right)-j N_{j}^{\mathcal{H}}\left(B_{m-1}\right)}{N-m+1} .
$$

We will use this expression to find a suitable expression for $D_{j}^{\mathcal{H}}\left(B_{m}\right)$ in terms of the deviations $D_{j-1}^{\mathcal{H}}\left(B_{m-1}\right), D_{j}^{\mathcal{H}}\left(B_{m-1}\right)$ and $X_{j}^{\mathcal{H}}\left(B_{m}\right)$. The first step will be to express $D_{j}$ as $N_{j}-L_{j}$. To reach the later steps we expand $N_{j}^{\mathcal{H}}\left(B_{m}\right)$ as $A_{j}^{\mathcal{H}}\left(B_{m}\right)+N_{j}^{\mathcal{H}}\left(B_{m-1}\right)$ and when possible express $N_{j}$ as $L_{j}+D_{j}$ and use the identity

$$
\frac{(k-j+1) L_{j-1}^{\mathcal{H}}(m-1)-j L_{j}^{\mathcal{H}}(m-1)}{N-m+1}=L_{j}^{\mathcal{H}}(m)-L_{j}^{\mathcal{H}}(m-1) .
$$

We obtain the following expression for $D_{j}^{\mathcal{H}}\left(B_{m}\right)$ :

$$
\begin{aligned}
D_{j}^{\mathcal{H}}\left(B_{m}\right) & =N_{j}^{\mathcal{H}}\left(B_{m}\right)-L_{j}^{\mathcal{H}}(m) \\
& =A_{j}^{\mathcal{H}}\left(B_{m}\right)+D_{j}^{\mathcal{H}}\left(B_{m-1}\right)-\left(L_{j}^{\mathcal{H}}(m)-L_{j}^{\mathcal{H}}(m-1)\right) \\
& =\frac{N-m-j+1}{N-m+1} D_{j}^{\mathcal{H}}\left(B_{m-1}\right)+\frac{k-j+1}{N-m+1} D_{j-1}^{\mathcal{H}}\left(B_{m-1}\right)+X_{j}^{\mathcal{H}}\left(B_{m}\right),
\end{aligned}
$$

where in the last step we used $A_{j}^{\mathcal{H}}\left(B_{m}\right)=X_{j}^{\mathcal{H}}\left(B_{m}\right)+\mathbb{E}\left[A_{j}^{\mathcal{H}}\left(B_{m}\right) \mid B_{m-1}\right],(3-3)$, and (3-4). The required expression (3-2) now follows immediately from the induction hypothesis by simply checking the coefficient of each $X_{\ell}^{\mathcal{H}}\left(B_{i}\right)$. This may be verified easily by checking the cases (i) $i=m$ and $\ell=j$, (ii) $i=m$ and $\ell<j$, (iii) $i<m$ and $\ell=j$, and (iv) $i<m$ and $\ell<j$.

In case (i), the coefficients on each side are 1 and in case (ii), the coefficients on each side are 0 . In case (iii), the coefficients on both sides are $(N-m)_{j} /(N-i)_{j}$. 
Finally in case (iv), the equality of the coefficients follows from the identity

$$
(N-m-j+1)(m-i-j+\ell)+(N-i-j+1)(j-\ell)=(N-m-\ell+1)(m-i) .
$$

\section{3}

\section{Some useful probability results}

In this section we prove a variant of Hoeffding-Azuma inequality which is useful when we cannot bound the increments almost surely, but only with large probability. The proof of this variant relies on a "truncation" argument and on the following simple observation.

Lemma 3.2 Given a $B_{i}$-measurable event $E$, let $E^{-}$be the $B_{i-1}$-measurable event that $E$ occurs for some extension $b_{i}=x$ with $x \in[N] \backslash B_{i-1}$. Then

$$
\mathbb{P}(E) \leqslant \mathbb{P}\left(E^{-}\right) \leqslant N \mathbb{P}(E)
$$

Proof. We first observe that $E \subset E^{-}$. Moreover, since the conditional probability $\mathbb{P}\left(E \mid E^{-}\right)$is between $1 / N$ and 1 , we immediately obtain the result.

By considering a "truncation" of the increments in which $X_{i}$ is set to 0 if it could be larger than $c_{i}$ with positive probability then one obtains the following variant of Hoeffding-Azuma inequality (Lemma 2.12). We state the lemma in our context of a sequence of random sets $\left(B_{i}\right)_{i=0}^{m}$.

Lemma 3.3 (Hoeffding-Azuma inequality (a variant)) Let $\left(S_{i}\right)_{i=0}^{m}$ be a martingale with respect to the natural filtration of the process $\left(B_{i}\right)_{i=0}^{m}$, let $\left(X_{i}\right)_{i=1}^{m}$ be the increments of the process and let $\left(c_{i}\right)_{i=1}^{m}$ be a sequence of real numbers. Then, for each $a>0$,

$$
\mathbb{P}\left(S_{m}-S_{0}>a\right) \leqslant \exp \left(\frac{-a^{2}}{2 \sum_{i=1}^{m} c_{i}^{2}}\right)+N \sum_{i=1}^{m} \mathbb{P}\left(\left|X_{i}\right|>c_{i}\right)
$$

Furthermore, the same bound holds for $\mathbb{P}\left(S_{m}-S_{0}<-a\right)$.

Proof. We first define for $1 \leqslant i \leqslant m$ the "truncation" $X_{i}^{*}$ of the increment $X_{i}$ as

$$
X_{i}^{*}:=X_{i} 1_{\left\|X_{i} \mid B_{i-1}\right\|_{\infty} \leqslant c_{i}},
$$

where $\left\|X_{i} \mid B_{i-1}\right\|_{\infty}$ denotes the maximum possible value of $X_{i}$ over the possible choices $b_{i} \in[N] \backslash B_{i-1}$. Let us define a new process $\left(S_{j}^{*}\right)_{j=0}^{m}$ by $S_{0}^{*}:=S_{0}$ and 
for $1 \leqslant j \leqslant m$ :

$$
S_{j}^{*}:=S_{0}^{*}+\sum_{i=1}^{j} X_{i}^{*} .
$$

Since the event considered by the indicator function is $B_{i-1}-$ measurable we have $\mathbb{E}\left[X_{i}^{*} \mid B_{i-1}\right]=0$ and so $\left(S_{j}^{*}\right)_{j=0}^{m}$ is a martingale with respect to the natural filtration of the process $\left(B_{i}\right)_{i=0}^{m}$. Note also that the increments of this process satisfy $\left|X_{i}^{*}\right| \leqslant c_{i}$ almost surely. Therefore Hoeffding-Azuma inequality gives us

$$
\mathbb{P}\left(S_{m}^{*}-S_{0}^{*}>a\right) \leqslant \exp \left(\frac{-a^{2}}{2 \sum_{i=1}^{m} c_{i}^{2}}\right) .
$$

We also observe that by the union bound and Lemma 3.2

$$
\begin{aligned}
\mathbb{P}\left(S_{m}^{*} \neq S_{m}\right) & \leqslant \sum_{i=1}^{m} \mathbb{P}\left(X_{i}^{*} \neq X_{i}\right) \\
& =\sum_{i=1}^{m} \mathbb{P}\left(\left\|X_{i} \mid B_{i-1}\right\|_{\infty}>c_{i}\right) \\
& \leqslant N \sum_{i=1}^{m} \mathbb{P}\left(\left|X_{i}\right|>c_{i}\right) .
\end{aligned}
$$

Finally we have

$$
\mathbb{P}\left(S_{m}-S_{0}>a\right) \leqslant \mathbb{P}\left(S_{m}^{*}-S_{0}^{*}>a\right)+\mathbb{P}\left(S_{m}^{*} \neq S_{m}\right)
$$

and so by (3-5) and (3-6) we get the desired result. 


\section{4}

\section{Deviations in $\mathbf{m}$-model for regular hypergraphs}

In this chapter, we present the proof of Theorem 1.2, which we will restate for the convenience of the reader.

Theorem (Restatement of Theorem 1.2) Let $1 \leqslant r \leqslant k$ and let $\eta \in$ $\left[0,3^{-r+1}\right]$. Let $\mathcal{H}$ be a $k$-uniform hypergraph on $[N]$. Suppose that $\mathcal{H}$ is $(r-1, \eta)$-near-regular with maximum $r$-degree $\Delta_{r}$. Then

$$
\mathbb{P}\left(D^{\mathcal{H}}\left(B_{m}\right)>a\right) \leqslant N^{O_{k}(1)} \exp \left(\frac{-\Omega_{k}(1) a^{2 / r}}{m \Delta_{r}^{2 / r}}\right)
$$

for all

$$
a \geqslant(10 k !)^{10^{r}} e(\mathcal{H})\left(\frac{\eta m^{k-1}}{N^{k-1}}\right)^{r /(r-1)} .
$$

Furthermore, the same bounds apply to the corresponding negative deviations.

The proof of Theorem 1.2 is given by induction on $r$. In fact we prove two series of statements with a joint induction. Since $N_{k}^{\mathcal{H}}\left(B_{m}\right)=N^{\mathcal{H}}\left(B_{m}\right)$, it is clear that Theorem 1.2 is contained in the sequence of statements $P_{r}, r \geqslant 1$ formulated below. In the following, we recall that $t=m / N$ and $s=i / N$.

$P_{r}:$ For all $k \geqslant j \geqslant r$ and $\eta \in\left[0,3^{-r+1}\right]$, for all $k$-uniform hypergraphs $\mathcal{H}$ on $[N]$ which are $(r-1, \eta)$-near-regular with maximum $r$-degree $\Delta_{r}$, we have

$$
\mathbb{P}\left(D_{j}^{\mathcal{H}}\left(B_{m}\right)>a\right) \leqslant N^{O_{k}(1)} \exp \left(\frac{-\Omega_{k}(1) a^{2 / r}}{m \Delta_{r}^{2 / r}}\right)
$$

for all $0 \leqslant m \leqslant N$ and all $a \geqslant C_{r} \eta^{r /(r-1)} e(\mathcal{H}) t^{(j-1) r /(r-1)}$, where $C_{r}=$ $(10 k !)^{10^{r}}$.

Remark 4.1 For $r=1$, the above bound holds for all $a>0$.

The other sequence of statements $Q_{r}$ will be related to the behaviour of the random variables $X_{\ell}^{\mathcal{H}}\left(B_{i}\right)$ that occur in the martingale representation of $D_{j}^{\mathcal{H}}\left(B_{m}\right)$. We define $Q_{r}$ to be the following statement. 
$Q_{r}:$ For all $k \geqslant \ell \geqslant r+1$ and $\eta \in\left[0,3^{-r}\right]$, for all $k$-uniform hypergraphs $\mathcal{H}$ on $[N]$ which are $(r, \eta)$-near-regular with maximum $(r+1)$-degree $\Delta_{r+1}$, we have

$$
\mathbb{P}\left(\left|X_{\ell}^{\mathcal{H}}\left(B_{i}\right)\right|>\alpha\right) \leqslant N^{O_{k}(1)} \exp \left(\frac{-\Omega_{k}(1) \alpha^{2 / r}}{i \Delta_{r+1}^{2 / r}}\right)
$$

for all $0 \leqslant i \leqslant N$ and all $\alpha \geqslant D_{r} \eta s^{\ell-1} e(\mathcal{H}) / N$, where $D_{r}=(10 k !)^{10^{r}+10}$.

As seen in the sequence of statements $Q_{r}$, a major part of the proof of Theorem 1.2 involves controlling the size of the increments in the martingale representation. In some cases we can control $\left|X_{\ell}^{\mathcal{H}}\left(B_{i}\right)\right|$ directly and deterministically, which is made clear by the following lemma.

Lemma 4.2 Let $\mathcal{H}$ be a k-uniform hypergraph on $[N]$ which is $(r, \eta)$-nearregular. If $1 \leqslant \ell \leqslant r$, then

$$
\left|X_{\ell}^{\mathcal{H}}\left(B_{i}\right)\right| \leqslant \frac{2 \ell\left(\begin{array}{l}
k \\
\ell
\end{array}\right) \eta s^{\ell-1} e(\mathcal{H})}{N} .
$$

for all $0 \leqslant i \leqslant N$.

We first prove Lemma 4.2 in Section 4.1. The base case of the induction, $P_{1}$, is proved in Section 4.2 by a straightforward application of the HoeffdingAzuma inequality. We complete the proof by showing that $P_{r}$ implies $Q_{r}$ and $Q_{r}$ implies $P_{r+1}$. These proofs are given in Sections 4.3 and 4.4 respectively.

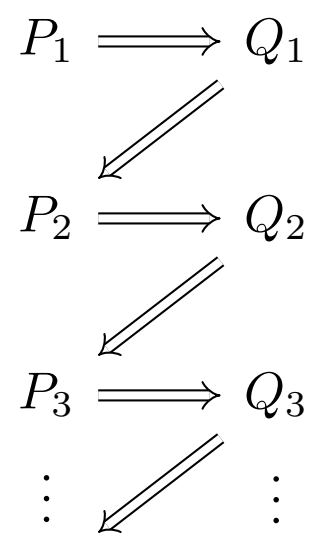

Figure 4.1: Illustration of the sequence of implications. 


\section{1}

\section{A deterministic control of the increments}

We now prove Lemma 4.2, which bounds the value of $\left|X_{\ell}^{\mathcal{H}}\left(B_{i}\right)\right|$ in a $k$-uniform $(r, \eta)$-near-regular hypergraph $\mathcal{H}$, for $1 \leqslant \ell \leqslant r$.

Proof of Lemma 4.2. Recall first that $X_{\ell}^{\mathcal{H}}\left(B_{i}\right):=A_{\ell}^{\mathcal{H}}\left(B_{i}\right)-\mathbb{E}\left[A_{\ell}^{\mathcal{H}}\left(B_{i}\right) \mid B_{i-1}\right]$ and that $A_{\ell}^{\mathcal{H}}\left(B_{i}\right)=N_{\ell}\left(B_{i}\right)-N_{\ell}\left(B_{i-1}\right)$. If $b_{i}$ is the last element added to $B_{i}$, i.e., $B_{i}=B_{i-1} \cup\left\{b_{i}\right\}$, then

$$
A_{\ell}^{\mathcal{H}}\left(B_{i}\right)=\sum_{\substack{C \subseteq B_{i-1} \\|C|=\ell-1}} d_{\mathcal{H}}\left(C \cup\left\{b_{i}\right\}\right)
$$

As $1 \leqslant \ell \leqslant r$ and $\mathcal{H}$ is $(r, \eta)$-near-regular, we have that $\mathcal{H}$ is also $(\ell, \eta)$-nearregular by part (i) of Lemma 2.4. And so $d_{\mathcal{H}}\left(C \cup\left\{b_{i}\right\}\right)=(1 \pm \eta) \bar{d}_{\ell}(\mathcal{H})$ for all $(\ell-1)$-element subsets $C \subseteq B_{i-1}$. It follows that

$$
A_{\ell}^{\mathcal{H}}\left(B_{i}\right)=(1 \pm \eta)\left(\begin{array}{l}
i-1 \\
\ell-1
\end{array}\right) \bar{d}_{\ell}(\mathcal{H}) \text {. }
$$

Now, as $X_{\ell}^{\mathcal{H}}\left(B_{i}\right):=A_{\ell}^{\mathcal{H}}\left(B_{i}\right)-\mathbb{E}\left[A_{\ell}^{\mathcal{H}}\left(B_{i}\right) \mid B_{i-1}\right]$, it follows that

$$
\left|X_{\ell}^{\mathcal{H}}\left(B_{i}\right)\right| \leqslant 2 \eta\left(\begin{array}{l}
i-1 \\
\ell-1
\end{array}\right) \bar{d}_{\ell}(\mathcal{H})
$$

Finally, since by Lemma 2.1, $\bar{d}_{\ell}(\mathcal{H})=e(\mathcal{H})\left(\begin{array}{l}k \\ \ell\end{array}\right) /\left(\begin{array}{l}N \\ \ell\end{array}\right)$, and using the bound $(i-1)_{\ell-1} \leqslant s^{\ell-1}(N-1)_{\ell-1}$, we obtain

$$
\left|X_{\ell}^{\mathcal{H}}\left(B_{i}\right)\right| \leqslant \frac{2 \ell\left(\begin{array}{l}
k \\
\ell
\end{array}\right) \eta s^{\ell-1} e(\mathcal{H})}{N}
$$

as required.

\section{2}

\section{The base case}

Let us fix $k \geqslant j \geqslant 1, \eta \in[0,1]$, and a $k$-uniform hypergraph $\mathcal{H}$ on $[N]$ with maximum degree $\Delta$.

We use the Hoeffding-Azuma inequality applied to the martingale representation:

$$
D_{j}^{\mathcal{H}}\left(B_{m}\right)=\sum_{i=1}^{m} \sum_{\ell=1}^{j} \frac{(N-m)_{\ell}(m-i)_{j-\ell}}{(N-i)_{j}} \cdot\left(\begin{array}{l}
k-\ell \\
k-j
\end{array}\right) X_{\ell}^{\mathcal{H}}\left(B_{i}\right) .
$$


In order to do so we must bound the magnitude of the increment

$$
Y_{i}:=\sum_{\ell=1}^{j} \frac{(N-m)_{\ell}(m-i)_{j-\ell}}{(N-i)_{j}} \cdot\left(\begin{array}{l}
k-\ell \\
k-j
\end{array}\right) X_{\ell}^{\mathcal{H}}\left(B_{i}\right)
$$

of the martingale. We observe that the first fraction is always at most 1 , and so the coefficient itself is $O_{k}(1)$. Recalling that $X_{\ell}^{\mathcal{H}}\left(B_{i}\right):=A_{\ell}^{\mathcal{H}}\left(B_{i}\right)-$ $\mathbb{E}\left[A_{\ell}^{\mathcal{H}}\left(B_{i}\right) \mid B_{i-1}\right]$, and that both $A_{\ell}^{\mathcal{H}}\left(B_{i}\right)$ and $\mathbb{E}\left[A_{\ell}^{\mathcal{H}}\left(B_{i}\right) \mid B_{i-1}\right]$ are non-negative we have

$$
\left\|X_{\ell}^{\mathcal{H}}\left(B_{i}\right)\right\|_{\infty} \leqslant\left\|A_{\ell}^{\mathcal{H}}\left(B_{i}\right)\right\|_{\infty} \leqslant O_{k}(1) \cdot \Delta \quad \text { a.s. }
$$

where the second inequality follows since $A_{\ell}^{\mathcal{H}}\left(B_{i}\right)=N_{\ell}^{\mathcal{H}}\left(B_{i}\right)-N_{\ell}^{\mathcal{H}}\left(B_{i-1}\right)$ is certainly at most $\left(\begin{array}{l}k-1 \\ \ell-1\end{array}\right)=O_{k}(1)$ times $d_{\mathcal{H}}\left(b_{i}\right) \leqslant \Delta$ (any "new" $\ell$-sets must be in edges containing $b_{i}$ ).

Since $Y_{i}$ consists of a finite number of terms and the coefficients are $O_{k}(1)$,

$$
\left|Y_{i}\right| \leqslant O_{k}(1) \cdot \Delta \quad \text { a.s. }
$$

By an application of the Hoeffding-Azuma inequality to $D_{j}^{\mathcal{H}}\left(B_{m}\right)=\sum_{i=1}^{m} Y_{i}$, we have that

$$
\mathbb{P}\left(D_{j}^{\mathcal{H}}\left(B_{m}\right)>a\right) \leqslant \exp \left(\frac{-a^{2}}{2 m O_{k}(1) \Delta^{2}}\right)=\exp \left(\frac{-\Omega_{k}(1) a^{2}}{m \Delta^{2}}\right) .
$$

This complete the proof of the base case $P_{1}$.

\section{3}

\section{$P_{r}$ implies $Q_{r}$}

In this section, we prove that $P_{r}$ implies $Q_{r}$. Let us fix $k \geqslant \ell \geqslant r+1$, $\eta \in\left[0,3^{-r}\right]$ and a $k$-uniform hypergraph $\mathcal{H}$ on $[N]$ which is $(r, \eta)$-near-regular. Let $\Delta_{r+1}$ be the maximum $(r+1)$-degree of $\mathcal{H}$. Let us also fix $0 \leqslant i \leqslant N$ and $\alpha \geqslant D_{r} \eta s^{\ell-1} e(\mathcal{H}) / N$. In order to prove $Q_{r}$ we must prove that

$$
\mathbb{P}\left(\left|X_{\ell}^{\mathcal{H}}\left(B_{i}\right)\right|>\alpha\right) \leqslant N^{O_{k}(1)} \exp \left(\frac{-\Omega_{k}(1) \alpha^{2 / r}}{i \Delta_{r+1}^{2 / r}}\right) .
$$

We recall that $X_{\ell}^{\mathcal{H}}\left(B_{i}\right)=A_{\ell}^{\mathcal{H}}\left(B_{i}\right)-\mathbb{E}\left[A_{\ell}^{\mathcal{H}}\left(B_{i}\right) \mid B_{i-1}\right]$. Our proof of (4-1) is based on the following proposition on the deviation of $A_{\ell}^{\mathcal{H}}\left(B_{i}\right)$ from its mean. We set

$$
\lambda_{\ell}(i):=\mathbb{E}\left[A_{\ell}^{\mathcal{H}}\left(B_{i}\right)\right]=\frac{\ell\left(\begin{array}{c}
k \\
\ell
\end{array}\right) e(\mathcal{H})(i-1)_{\ell-1}}{(N)_{\ell}} .
$$

Note that $\lambda_{\ell}\left(B_{i}\right)$ is also equal to $L_{\ell}(i)-L_{\ell}(i-1)$. 


\section{Proposition 4.3}

$$
\mathbb{P}\left(\left|A_{\ell}^{\mathcal{H}}\left(B_{i}\right)-\lambda_{\ell}(i)\right|>\alpha\right) \leqslant N^{O_{k}(1)} \exp \left(\frac{-\Omega_{k}(1) \alpha^{2 / r}}{i \Delta_{r+1}^{2 / r}}\right)
$$

for all $\alpha \geqslant D_{r} \eta s^{\ell-1} e(\mathcal{H}) / 2 N$.

Let us first observe that (4-1) follows from Proposition 4.3. Since $X_{\ell}^{\mathcal{H}}\left(B_{i}\right)=A_{\ell}^{\mathcal{H}}\left(B_{i}\right)-\mathbb{E}\left[A_{\ell}^{\mathcal{H}}\left(B_{i}\right) \mid B_{i-1}\right]$, the event $\left|X_{\ell}^{\mathcal{H}}\left(B_{i}\right)\right|>\alpha$ of (4-1) may only occur if either $\left|A_{\ell}^{\mathcal{H}}\left(B_{i}\right)-\lambda_{\ell}(i)\right|>\alpha / 2$ or $\left|\mathbb{E}\left[A_{\ell}^{\mathcal{H}}\left(B_{i}\right) \mid B_{i-1}\right]-\lambda_{\ell}(i)\right|>\alpha / 2$, by the triangle inequality. The first of these events has probability at most

$$
N^{O_{k}(1)} \exp \left(\frac{-\Omega_{k}(1) \alpha^{2 / r}}{i \Delta_{r+1}^{2 / r}}\right)
$$

by Proposition 4.3. The second may only occur if there exists $x \in[N] \backslash B_{i-1}$ such that $\left|A_{\ell}^{\mathcal{H}}\left(B_{i-1} \cup\{x\}\right)-\lambda_{\ell}(i)\right|>\alpha / 2$ and so the same bound holds by Lemma 3.2 and Proposition 4.3. This completes the proof of (4-1).

All that remains is to prove Proposition 4.3. We shall base the proof of Proposition 4.3 on Lemma 4.4, which shows how we may view $A_{\ell}$ in terms of a deviation in the link hypergraph $\mathcal{H}(x)$, and Lemma 4.5 which uses $P_{r}$ to bound the probability of such deviations.

It will be useful to condition on the element $b_{i}=x$ that is added as the $i$-th element of the process. Given that $b_{i}=x$ the set $B_{i}$ is distributed as

$$
B_{i}=B_{i-1}^{(x)} \cup\{x\}
$$

where $B_{i-1}^{(x)}$ is a uniformly random subset of $i-1$ elements of $[N] \backslash\{x\}$. We also recall that we defined the link hypergraph of $\mathcal{H}$ with respect to $x$ as

$$
V(\mathcal{H}(x))=V(\mathcal{H}) \backslash\{x\}, E(\mathcal{H}(x)):=\{f \backslash\{x\}: f \in E(\mathcal{H}), x \in f\}
$$

The first lemma shows that $A_{\ell}^{\mathcal{H}}\left(B_{i-1}^{(x)} \cup\{x\}\right)$ may be expressed precisely in terms of the deviation of $(\ell-1)$ sets in the hypergraph $\mathcal{H}(x)$.

Lemma 4.4 For each $x \in[N]$

$$
\begin{aligned}
A_{\ell}^{\mathcal{H}}\left(B_{i-1}^{(x)} \cup\{x\}\right) & =D_{\ell-1}^{\mathcal{H}(x)}\left(B_{i-1}^{(x)}\right)+\mathbb{E}\left[N_{\ell-1}^{\mathcal{H}(x)}\left(B_{i-1}^{(x)}\right)\right] \\
& =D_{\ell-1}^{\mathcal{H}(x)}\left(B_{i-1}^{(x)}\right)+\lambda_{\ell}(i) \pm \frac{\eta \ell\left(\begin{array}{l}
k \\
\ell
\end{array}\right) e(\mathcal{H}) s^{\ell-1}}{N} .
\end{aligned}
$$

We use $P_{r}$ to provide a bound on the probability that $D_{\ell-1}^{\mathcal{H}(x)}\left(B_{i-1}^{(x)}\right)$ is large. 
Lemma 4.5 Let $r+1 \leqslant \ell \leqslant k$. Then

$$
\mathbb{P}\left(\left|D_{\ell-1}^{\mathcal{H}(x)}\left(B_{i-1}^{(x)}\right)\right|>\alpha\right) \leqslant N^{O_{k}(1)} \exp \left(\frac{-\Omega_{k}(1) \alpha^{2 / r}}{i \Delta_{r+1}^{2 / r}}\right)
$$

for all $\alpha \geqslant C_{r}(3 \eta)^{r /(r-1)} e(\mathcal{H}(x)) s^{(\ell-2) r /(r-1)}$. In particular, the result holds for all $\alpha \geqslant D_{r} \eta s^{\ell-1} e(\mathcal{H}) / 4 N$.

Remark 4.6 For $r=1$, the above bound holds for all $\alpha>0$, since the bound in $P_{1}$ holds for all $a>0$.

Let us see how Proposition 4.3 follows from these lemmas.

Proof of Proposition 4.3. By Lemma 4.4, conditioned on $b_{i}=x$, we have that

$$
\left|A_{\ell}^{\mathcal{H}}\left(B_{i}\right)-\lambda_{\ell}(i)\right| \leqslant\left|D_{\ell-1}^{\mathcal{H}(x)}\left(B_{i-1}^{(x)}\right)\right|+\frac{\eta \ell\left(\begin{array}{l}
k \\
\ell
\end{array}\right) e(\mathcal{H}) s^{\ell-1}}{N} .
$$

Since the second term on the right is at most $\alpha / 2$ (this follows from the condition on $\alpha$ and the fact that $\left.D_{r} \geqslant 4 \ell\left(\begin{array}{l}k \\ \ell\end{array}\right)\right)$, the event that $\left|A_{\ell}^{\mathcal{H}}\left(B_{i}\right)-\lambda_{\ell}(i)\right|>$ $\alpha$ is contained in the event that $\left|D_{\ell-1}^{\mathcal{H}(x)}\left(B_{i-1}^{(x)}\right)\right|>\alpha / 2$. The required bound now follows immediately from Lemma 4.5 and hence also unconditionally.

All that remains is to prove Lemma 4.4 and Lemma 4.5. We begin with Lemma 4.4.

Proof of Lemma 4.4. Recall that

$$
A_{\ell}^{\mathcal{H}}\left(B_{i-1}^{(x)} \cup\{x\}\right):=N_{\ell}^{\mathcal{H}}\left(B_{i-1}^{(x)} \cup\{x\}\right)-N_{\ell}^{\mathcal{H}}\left(B_{i-1}^{(x)}\right)
$$

which is precisely the number of pairs $(S, f)$ where $S \subseteq B_{i-1}^{(x)}$ is a subset of $\ell-1$ elements, and $f$ is an edge of $\mathcal{H}$ such that $S \cup\{x\} \subseteq f$. Setting $f^{-}=f \backslash\{x\}$ we note that this condition is equivalent to the fact that $S \subseteq f^{-}$. It follows that $A_{\ell}^{\mathcal{H}}\left(B_{i-1}^{(x)} \cup\{x\}\right)$ is precisely $N_{\ell-1}^{\mathcal{H}(x)}\left(B_{i-1}^{(x)}\right)$, and so

$$
A_{\ell}^{\mathcal{H}}\left(B_{i-1}^{(x)} \cup\{x\}\right)=D_{\ell-1}^{\mathcal{H}(x)}\left(B_{i-1}^{(x)}\right)+\mathbb{E}\left[N_{\ell-1}^{\mathcal{H}(x)}\left(B_{i-1}^{(x)}\right)\right]
$$

by the definition of $D_{\ell-1}^{\mathcal{H}(x)}\left(B_{i-1}^{(x)}\right)$ as the deviation of $N_{\ell-1}^{\mathcal{H}(x)}\left(B_{i-1}^{(x)}\right)$ from its mean. All that remains is to prove that

$$
\mathbb{E}\left[N_{\ell-1}^{\mathcal{H}(x)}\left(B_{i-1}^{(x)}\right)\right]=\lambda_{\ell}(i) \pm \frac{\eta \ell\left(\begin{array}{l}
k \\
\ell
\end{array}\right) s^{\ell-1} e(\mathcal{H})}{N} .
$$

Since $\mathcal{H}$ is $(r, \eta)$-near-regular (and so $(1, \eta)$-near-regular by Lemma 2.4 ) we have $e(\mathcal{H}(x))=d_{\mathcal{H}}(x)=(1 \pm \eta) e(\mathcal{H}) k / N$ by Lemma 2.1. We may also observe 
that $\mathcal{H}(x)$ is $(k-1)$-uniform on $N-1$ vertices and so

$$
\begin{aligned}
\mathbb{E}\left[N_{\ell-1}^{\mathcal{H}(x)}\left(B_{i-1}^{(x)}\right)\right] & =\frac{e(\mathcal{H}(x))\left(\begin{array}{c}
k-1 \\
\ell-1
\end{array}\right)(i-1)_{\ell-1}}{(N-1)_{\ell-1}} \\
& =\frac{\ell\left(\begin{array}{l}
k \\
\ell
\end{array}\right) e(\mathcal{H})(i-1)_{\ell-1}}{(N)_{\ell}} \pm \frac{\eta \ell\left(\begin{array}{l}
k \\
\ell
\end{array}\right) e(\mathcal{H})(i-1)_{\ell-1}}{(N)_{\ell}} \\
& =\lambda_{\ell}(i) \pm \frac{\eta \ell\left(\begin{array}{l}
k \\
\ell
\end{array}\right) s^{\ell-1} e(\mathcal{H})}{N}
\end{aligned}
$$

as required.

We now prove Lemma 4.5.

Proof of Lemma 4.5. We prove the required bound by applying the inequality given by $P_{r}$ to the hypergraph $\mathcal{H}(x)$. We observe that $\mathcal{H}(x)$ is a $(k-1)$-uniform hypergraph on $N-1$ vertices. We may also observe that $\mathcal{H}(x)$ inherits the regularity condition $(r-1,3 \eta)$-near-regular from $\mathcal{H}$ by Lemma 2.4 and the maximum $r$-degree of $\mathcal{H}(x)$ is at most $\Delta_{r+1}$. By $P_{r}$ we have that

$$
\mathbb{P}\left(\left|D_{\ell-1}^{\mathcal{H}(x)}\left(B_{i-1}^{(x)}\right)\right|>\alpha\right) \leqslant N^{O_{k}(1)} \exp \left(\frac{-\Omega_{k}(1) \alpha^{2 / r}}{i \Delta_{r+1}^{2 / r}}\right)
$$

for all $\alpha \geqslant C_{r}(3 \eta)^{r /(r-1)} e(\mathcal{H}(x)) s^{(\ell-2) r /(r-1)}$. This is exactly the result we need. All that remains is to verify that this includes all $\alpha \geqslant D_{r} \eta s^{\ell-1} e(\mathcal{H}) / 4 N$.

We have that $D_{r} \geqslant 10 k \cdot 3^{r /(r-1)} C_{r}, \eta<1,(\ell-2) r \geqslant(\ell-1)(r-1)$ and $e(\mathcal{H}(x)) \leqslant(1+\eta) e(\mathcal{H}) k / N \leqslant 2 e(\mathcal{H}) k / N$. It follows that

$$
\begin{aligned}
\frac{D_{r} \eta e(\mathcal{H}) s^{\ell-1}}{4 N} & \geqslant \frac{10 C_{r}(3 \eta)^{r /(r-1)} e(\mathcal{H}) k s^{(\ell-2) r /(r-1)}}{4 N} \\
& \geqslant C_{r}(3 \eta)^{r /(r-1)} e(\mathcal{H}(x)) s^{(\ell-2) r /(r-1)}
\end{aligned}
$$

This confirms that the inequality holds across the whole of the range we claimed.

\section{4}

$Q_{r}$ implies $P_{r+1}$

In this section, we prove that $Q_{r}$ implies $P_{r+1}$. The main idea of the induction step is using our information about the magnitude of increments combined with the variant of the Hoeffding-Azuma inequality, Lemma 3.3. Let us fix $k \geqslant j \geqslant r+1, \eta \in\left[0,3^{-r}\right]$ and a $k$-uniform $(r, \eta)$-near-regular hypergraph $\mathcal{H}$ on $[N]$ with maximum $(r+1)$-degree $\Delta_{r+1}$. We recall the 
martingale representation

$$
D_{j}^{\mathcal{H}}\left(B_{m}\right)=\sum_{i=1}^{m} Y_{i}
$$

where

$$
Y_{i}=\sum_{\ell=1}^{j} \frac{(N-m)_{\ell}(m-i)_{j-\ell}}{(N-i)_{j}}\left(\begin{array}{l}
k-\ell \\
k-j
\end{array}\right) X_{\ell}^{\mathcal{H}}\left(B_{i}\right) .
$$

We now prove an auxiliary lemma that controls the probability that the increments are large.

Lemma 4.7 If $\alpha \geqslant j k ! D_{r} \eta t^{j-1} e(\mathcal{H}) / N$, then

$$
\mathbb{P}\left(\left|Y_{i}\right|>\alpha\right) \leqslant N^{O_{k}(1)} \exp \left(\frac{-\Omega_{k}(1) \alpha^{2 / r}}{m \Delta_{r+1}^{2 / r}}\right) .
$$

Proof. We first observe that

$$
\left|Y_{i}\right| \leqslant \sum_{\ell=1}^{j} k ! t^{j-\ell}\left|X_{\ell}^{\mathcal{H}}\left(B_{i}\right)\right|
$$

Indeed, we have $\left(\begin{array}{l}k-\ell \\ k-j\end{array}\right) \leqslant k !$ and

$$
\begin{aligned}
\frac{(N-m)_{\ell}(m-i)_{j-\ell}}{(N-i)_{j}} & =\frac{(N-m)_{\ell}}{(N-i-j+\ell)_{\ell}} \cdot \frac{(m-i)_{j-\ell}}{(N-i)_{j-\ell}} \\
& \leqslant t^{j-\ell}
\end{aligned}
$$

since the first fraction is at most 1 (because $m-i \geqslant j-\ell$, otherwise $(m-i)_{j-\ell}$ would be 0 and the inequality would be trivial) and the second fraction is at $\operatorname{most}(m / N)^{j-\ell}=t^{j-\ell}$.

By an application of Lemma 4.2 and $Q_{r}$, since $\alpha \geqslant j k ! D_{r} \eta t^{j-1} e(\mathcal{H}) / N$ the following bound holds for all $1 \leqslant \ell \leqslant k$ :

$$
\mathbb{P}\left(\left|X_{\ell}^{\mathcal{H}}\left(B_{i}\right)\right|>\frac{\alpha t^{\ell-j}}{j k !}\right) \leqslant N^{O_{k}(1)} \exp \left(\frac{-\Omega_{k}(1) \alpha^{2 / r}}{m \Delta_{r+1}^{2 / r}}\right) .
$$

Finally, using (4-2), (4-3) and the union bound, we have

$$
\mathbb{P}\left(\left|Y_{i}\right|>\alpha\right) \leqslant N^{O_{k}(1)} \exp \left(\frac{-\Omega_{k}(1) \alpha^{2 / r}}{m \Delta_{r+1}^{2 / r}}\right) .
$$

We are now ready to prove $P_{r+1}$. Let $a \geqslant C_{r+1} \eta^{(r+1) / r} e(\mathcal{H}) t^{(j-1)(r+1) / r}$. Choosing $\alpha=a^{r /(r+1)} \Delta_{r+1}^{1 /(r+1)}$, we can easily verify that

$$
\alpha \geqslant j k ! D_{r} \eta t^{j-1} e(\mathcal{H}) / N
$$


using $\Delta_{r+1} \geqslant \bar{d}_{r+1}(\mathcal{H}) \geqslant e(\mathcal{H}) /\left(N^{r+1}\right)$, by Lemma 2.1 , and $C_{r+1} \geqslant k^{2}(k !)^{2} D_{r}^{2}$. By an application of the Hoeffding-Azuma inequality (the version given in Lemma 3.3) with $c_{i}=\alpha$ for every $i$, we have

$$
\mathbb{P}\left(D_{j}^{\mathcal{H}}\left(B_{m}\right)>a\right) \leqslant \exp \left(\frac{-a^{2}}{2 m \alpha^{2}}\right)+N \sum_{i=1}^{m} \mathbb{P}\left(\left|Y_{i}\right|>\alpha\right) .
$$

By Lemma 4.7, we obtain

$$
\mathbb{P}\left(D_{j}^{\mathcal{H}}\left(B_{m}\right)>a\right) \leqslant \exp \left(\frac{-a^{2}}{2 m \alpha^{2}}\right)+N^{O_{k}(1)} \exp \left(\frac{-\Omega_{k}(1) \alpha^{2 / r}}{m \Delta_{r+1}^{2 / r}}\right)
$$

Since $\alpha=a^{r /(r+1)} \Delta_{r+1}^{1 /(r+1)}$, this last inequality gives us

$$
\mathbb{P}\left(D_{j}^{\mathcal{H}}\left(B_{m}\right)>a\right) \leqslant N^{O_{k}(1)} \exp \left(\frac{-\Omega_{k}(1) a^{2 /(r+1)}}{m \Delta_{r+1}^{2 /(r+1)}}\right)
$$

which establishes the induction step.

Now that we have established the base case $P_{1}$ and the implications $P_{r} \Rightarrow Q_{r}$ and $Q_{r} \Rightarrow P_{r+1}$ for all $r \geqslant 1$ we have completed the proof of Theorem 1.2. 


\section{5}

\section{Deviations in $\mathbf{p}$-model for regular hypergraphs}

In this chapter, we prove Theorem 1.5. We restate the theorem below for the convenience of the reader.

Theorem (Restatement of Theorem 1.5) Let $k \geqslant r \geqslant 2$. Let $\mathcal{H}_{N}$ be a sequence of $k$-uniform hypergraphs which are $(r-1, \eta)$-near-regular with maximum $r$-degree $\Delta_{r}=\Delta_{r}\left(\mathcal{H}_{N}\right)$ and $V\left(\mathcal{H}_{N}\right)=[N]$. Let $\delta_{N}$ be a sequence satisfying

$$
\max \left\{\frac{\Delta_{r}(N \log N)^{r / 2}}{p^{k-r / 2} e\left(\mathcal{H}_{N}\right)},\left(\eta^{r} p^{k-r}\right)^{1 /(r-1)}, \frac{1}{\sqrt{p N}}\right\} \ll \delta_{N} \ll\left(\frac{p^{k-r} e\left(\mathcal{H}_{N}\right)}{N^{r} \Delta_{r}}\right)^{1 /(r-1)},
$$

where $p \leqslant 1$ is bounded away from 1 . Then

$$
\mathbb{P}\left(D^{\mathcal{H}_{N}}\left(B_{p}\right)>\delta_{N} L^{\mathcal{H}_{N}}(p)\right)=\exp \left(-(1+o(1)) \frac{\delta_{N}^{2} p N}{2 k^{2}(1-p)}\right) .
$$

Furthermore, the same bounds apply to the corresponding negative deviations.

The idea of the proof is that deviations $D\left(B_{p}\right)$ in the $p$-model are intimately related to deviations $D\left(B_{m}\right)$ in the $m$-model, via the identity

$$
\mathbb{P}\left(D^{\mathcal{H}_{N}}\left(B_{p}\right)>a\right)=\sum_{m=0}^{N} b_{N, p}(m) \mathbb{P}\left(N^{\mathcal{H}_{N}}\left(B_{m}\right)>p^{k} e\left(\mathcal{H}_{N}\right)+a\right),
$$

where $b_{N, p}(m):=\mathbb{P}(\operatorname{Bin}(N, p)=m)$ and $\operatorname{Bin}(N, p)$ denotes the binomial distribution with parameters $N$ and $p$. Recall that $L^{\mathcal{H}_{N}}(p)=p^{k} e\left(\mathcal{H}_{N}\right)$ is the expected value of $N^{\mathcal{H}_{N}}\left(B_{p}\right)$ and we study the probability that the deviation satisfies $D^{\mathcal{H}_{N}}\left(B_{p}\right)>\delta_{N} L^{\mathcal{H}_{N}}(p)$.

Our proof of Theorem 1.5 consists of a lower bound (see Section 5.1) and an upper bound (see Section 5.2). The lower bound is based on the fact that $\mathbb{P}\left(N^{\mathcal{H}_{N}}\left(B_{m}\right)>p^{k} e\left(\mathcal{H}_{N}\right)+a\right)$ is increasing in $m$ and so $\mathbb{P}\left(D^{\mathcal{H}_{N}}\left(B_{p}\right)>\delta_{N} L^{\mathcal{H}_{N}}(p)\right) \geqslant B_{N, p}\left(m_{+}\right) \mathbb{P}\left(N^{\mathcal{H}_{N}}\left(B_{m_{+}}\right)>\left(1+\delta_{N}\right) L^{\mathcal{H}_{N}}(p)\right)$ 
for all $m_{+} \geqslant 0$, where $B_{N, p}(m):=\mathbb{P}(\operatorname{Bin}(N, p) \geqslant m)$. For a particular choice of $m_{+}=m_{+}\left(\delta_{N}\right)$ we shall prove that

$$
B_{N, p}\left(m_{+}\right) \mathbb{P}\left(N^{\mathcal{H}_{N}}\left(B_{m_{+}}\right)>\left(1+\delta_{N}\right) L(p)\right)=\exp \left(-(1+o(1)) \frac{\delta_{N}^{2} p N}{2 k^{2}(1-p)}\right)
$$

which gives the required lower bound.

We need to work harder to prove the upper bound. We must control all contributions to the sum (5-1). We again use that $\mathbb{P}\left(N^{\mathcal{H}_{N}}\left(B_{m}\right)>p^{k} e\left(\mathcal{H}_{N}\right)+a\right)$ is increasing to observe that

$$
\mathbb{P}\left(D^{\mathcal{H}_{N}}\left(B_{p}\right)>\delta_{N} L(p)\right) \leqslant \mathbb{P}\left(N^{\mathcal{H}_{N}}\left(B_{m_{-}}\right)>\left(1+\delta_{N}\right) L(p)\right)+B_{N, p}\left(m_{-}\right)
$$

for all $m_{-} \geqslant 0$. We shall then choose $m_{-}=m_{-}\left(\delta_{N}\right)$ such that

$$
B_{N, p}\left(m_{-}\right)=\exp \left(-(1+o(1)) \frac{\delta_{N}^{2} p N}{2 k^{2}(1-p)}\right)
$$

and

$$
\mathbb{P}\left(N^{\mathcal{H}_{N}}\left(B_{m_{-}}\right)>\left(1+\delta_{N}\right) L(p)\right) \ll \exp \left(\frac{-\delta_{N}^{2} p N}{2 k^{2}(1-p)}\right) .
$$

The latter inequality is proved using Theorem 1.2.

Based on the above sketch it is clear that the probabilities $b_{N, p}(m)$ and $B_{N, p}(m)$, related to the binomial distribution, are central to our proof. While more precise estimates, up to a multiplicative factor of $1+o(1)$ are known (see Theorem 2.17 in Chapter 2), the following corollary is sufficient to our purposes.

Corollary 5.1 Suppose that $\left(x_{N}\right)$ is a sequence such that $1 \ll x_{N} \ll \sqrt{N p q}$.

Then

$$
b_{N, p}\left(\left\lfloor p N+x_{N} \sqrt{N p q}\right\rfloor\right)=\frac{1}{\sqrt{N p q}} \exp \left(-(1+o(1)) \frac{x_{N}^{2}}{2}\right)
$$

and

$$
B_{N, p}\left(\left\lfloor p N+x_{N} \sqrt{N p q}\right\rfloor\right)=\exp \left(-(1+o(1)) \frac{x_{N}^{2}}{2}\right)
$$

Both of the values $m_{-}$and $m_{+}$discussed above will be chosen in relation to

$$
m_{*}:=\left(1+\delta_{N}\right)^{1 / k} p N,
$$

which is chosen so that $L^{\mathcal{H}_{N}}\left(m_{*}\right)$ is approximately equal to $\left(1+\delta_{N}\right) L^{\mathcal{H}_{N}}(p)$. Let us also define

$$
x(m):=\frac{m-p N}{\sqrt{N p q}}
$$


in general and, in particular, set $x_{*}:=x\left(m_{*}\right)$.

\section{1}

\section{Lower Bound}

First we choose a sequence $f_{N}$ such that

$$
\max \left\{\eta^{r /(r-1)} p^{(k-1) /(r-1)} N, \frac{p^{r / 2-k+1} N^{r / 2+1} \Delta_{r}(\log N)^{r / 2}}{e\left(\mathcal{H}_{N}\right)}\right\} \ll f_{N} \ll \delta_{N} p N .
$$

Note that since

$$
\delta_{N} \gg \max \left\{\frac{\Delta_{r}(N \log N)^{r / 2}}{p^{k-r / 2} e\left(\mathcal{H}_{N}\right)},\left(\eta^{r} p^{k-r}\right)^{1 /(r-1)}, \frac{1}{\sqrt{p N}}\right\}
$$

there exists such sequence. We then choose $m_{+}=m_{*}+f_{N}$ and we also set $x_{+}=x\left(m_{+}\right)$.

Now we prove the following two lemmas, which together with (5-2), will give us the desired lower bound.

\section{Lemma 5.2}

$$
\mathbb{P}\left(N^{\mathcal{H}_{N}}\left(B_{m_{+}}\right)>\left(1+\delta_{N}\right) L^{\mathcal{H}_{N}}(p)\right)=1-o(1) .
$$

Proof. Note first that it suffices to prove that

$$
\mathbb{P}\left(D^{\mathcal{H}_{N}}\left(B_{m_{+}}\right) \leqslant\left(1+\delta_{N}\right) L^{\mathcal{H}_{N}}(p)-L^{\mathcal{H}_{N}}\left(m_{+}\right)\right)=o(1) .
$$

Observe now that for sufficiently large $N$, we have

$$
\begin{aligned}
\left(1+\delta_{N}\right) L^{\mathcal{H}_{N}}(p)-L^{\mathcal{H}_{N}}\left(m_{+}\right) & =e\left(\mathcal{H}_{N}\right)\left[\left(1+\delta_{N}\right) p^{k}-\frac{\left(m_{+}\right)_{k}}{(N)_{k}}\right] \\
& \leqslant e\left(\mathcal{H}_{N}\right)\left[\left(1+\delta_{N}\right) p^{k}-\frac{\left(m_{*}+f_{N}-k\right)^{k}}{N^{k}}\right] \\
& \leqslant e\left(\mathcal{H}_{N}\right)\left[\left(1+\delta_{N}\right) p^{k}-\frac{m_{*}^{k}}{N^{k}}-\frac{m_{*}^{k-1} f_{N}}{N^{k}}\right] \\
& \leqslant-\frac{e\left(\mathcal{H}_{N}\right) p^{k-1} f_{N}}{N} .
\end{aligned}
$$

So we obtain

$$
\begin{aligned}
& \mathbb{P}\left(D^{\mathcal{H}_{N}}\left(B_{m_{+}}\right) \leqslant\left(1+\delta_{N}\right) L^{\mathcal{H}_{N}}(p)-L^{\mathcal{H}_{N}}\left(m_{+}\right)\right) \leqslant \\
& \mathbb{P}\left(D^{\mathcal{H}_{N}}\left(B_{m_{+}}\right) \leqslant-\frac{e\left(\mathcal{H}_{N}\right) p^{k-1} f_{N}}{N}\right) .
\end{aligned}
$$


Let us denote $a=\left(e\left(\mathcal{H}_{N}\right) p^{k-1} f_{N}\right) / N$. Since $f_{N} \gg \eta^{r /(r-1)} p^{(k-1) /(r-1)} N$, if $N$ is sufficiently large, we can apply Theorem 1.2 and this gives us

$$
\mathbb{P}\left(D^{\mathcal{H}_{N}}\left(B_{m_{+}}\right) \leqslant-a\right) \leqslant N^{O_{k}(1)} \exp \left(\frac{-\Omega_{k}(1) a^{2 / r}}{m_{+} \Delta_{r}^{2 / r}}\right) .
$$

Using now that

$$
f_{N} \gg \frac{p^{r / 2-k+1} N^{r / 2+1} \Delta_{r}(\log N)^{r / 2}}{e\left(\mathcal{H}_{N}\right)}
$$

we can easily verify that

$$
N^{O_{k}(1)} \exp \left(\frac{-\Omega_{k}(1) a^{2 / r}}{m_{+} \Delta_{r}^{2 / r}}\right)=o(1),
$$

which gives us the desired result.

\section{Lemma 5.3}

$$
B_{N, p}\left(m_{+}\right)=\exp \left(-(1+o(1)) \frac{\delta_{N}^{2} p N}{2 k^{2}(1-p)}\right)
$$

Proof. By Corollary 5.1, we have

$$
B_{N, p}\left(m_{+}\right)=\exp \left(-(1+o(1)) \frac{x_{+}^{2}}{2}\right)
$$

Observe now that

$$
\frac{x_{+}}{x_{*}}=1+\frac{f_{N}}{p N\left[\left(1+\delta_{N}\right)^{1 / k}-1\right]} .
$$

Since $\delta_{N} \ll 1,\left(1+\delta_{N}\right)^{1 / k}-1=\Theta\left(\delta_{N}\right)$ and as $f_{N} \ll \delta_{N} p N$, we obtain $x_{+}=(1+o(1)) x_{*}$, which gives us

$$
B_{N, p}\left(m_{+}\right)=\exp \left(-(1+o(1)) \frac{x_{*}^{2}}{2}\right)
$$

Finally, note that

$$
x_{*}=\sqrt{\frac{p N}{1-p}}\left(\left(1+\delta_{N}\right)^{1 / k}-1\right)
$$

and since $\left(1+\delta_{N}\right)^{1 / k}-1=(1+o(1)) \delta_{N} / k$, we obtain the required result. 


\section{2}

\section{Upper Bound}

Our upper bound on $\mathbb{P}\left(D^{\mathcal{H}_{N}}\left(B_{p}\right)>\delta_{N} L^{\mathcal{H}_{N}}(p)\right)$ requires us to control all the terms of the sum (5-1), i.e., all the terms in the sum

$$
\begin{aligned}
& \mathbb{P}\left(D^{\mathcal{H}_{N}}\left(B_{p}\right)>\delta_{N} L^{\mathcal{H}_{N}}(p)\right) \\
& =\sum_{m=0}^{N} b_{N, p}(m) \mathbb{P}\left(N^{\mathcal{H}_{N}}\left(B_{m}\right)>p^{k} e\left(\mathcal{H}_{N}\right)+\delta_{N} L^{\mathcal{H}_{N}}(p)\right) .
\end{aligned}
$$

In fact we do not require a very precise analysis. We shall simply break the sum into two parts $m<m_{-}$and $m \geqslant m_{-}$for a value of $m_{-}$which we define below. We bound the terms $m \geqslant m_{-}$using only the first probability (the binomial) and the terms $m<m_{-}$using only the second probability (the deviation in the model $\left.B_{m}\right)$.

Let $g_{N}$ be a sequence satisfying

$$
\max \left\{\frac{\eta^{r /(r-1)} p^{(k-1) /(r-1)} N}{e\left(\mathcal{H}_{N}\right)}, \frac{N^{r / 2+1}(\log N)^{r / 2} \Delta_{r}}{p^{k-r / 2-1} e\left(\mathcal{H}_{N}\right)}, \frac{\delta_{N}^{r} N^{r+1} \Delta_{r}}{p^{k-r-1} e\left(\mathcal{H}_{N}\right)}\right\} \ll g_{N} \ll \delta_{N} p N
$$

It is certainly possible to choose such a sequence by the conditions on $\delta_{N}$ in Theorem 1.5.

We define $m_{-}:=m_{*}-g_{N}$ and set $x_{-}=x\left(m_{-}\right)$. By a calculation similar to that given in the proof of Lemma 5.2 we have

$$
L^{\mathcal{H}_{N}}\left(m_{-}\right) \leqslant\left(1+\delta_{N}\right) L^{\mathcal{H}_{N}}(p)-\frac{e\left(\mathcal{H}_{N}\right) p^{k-1} g_{N}}{N} .
$$

We now bound the two parts of the sum. First, for the part $m \geqslant m_{-}$we simply use that the sum of these terms is at most

$$
B_{N, p}\left(m_{-}\right)=\exp \left(-(1+o(1)) \frac{\delta_{N}^{2} p N}{2 k^{2}(1-p)}\right)
$$

This may be verified by simply following the proof of Lemma 5.3 and using that $g_{N} \ll \delta_{N} p N$.

Now, we bound the rest of the sum by $\exp \left(-x_{*}^{2}\right)$ using the following lemma. This will complete the proof of the upper bound.

\section{Lemma 5.4}

$$
\sum_{m=0}^{m_{-}} \mathbb{P}\left(N^{\mathcal{H}_{N}}\left(B_{m}\right)>p^{k} e\left(\mathcal{H}_{N}\right)+\delta_{N} L^{\mathcal{H}_{N}}(p)\right) \leqslant \exp \left(-x_{*}^{2}\right) .
$$

Proof. Since $\mathbb{P}\left(N^{\mathcal{H}_{N}}\left(B_{m}\right)>p^{k} e\left(\mathcal{H}_{N}\right)+\delta_{N} L^{\mathcal{H}_{N}}(p)\right)$ is increasing in $m$ it suffices to prove that 


$$
\mathbb{P}\left(N^{\mathcal{H}_{N}}\left(B_{m_{-}}\right)>p^{k} e\left(\mathcal{H}_{N}\right)+\delta_{N} L^{\mathcal{H}_{N}}(p)\right) \leqslant \frac{\exp \left(-x_{*}^{2}\right)}{N} .
$$

By (5-3) this event is contained in the event that $D^{\mathcal{H}_{N}}\left(B_{m_{-}}\right)>$ $e\left(\mathcal{H}_{N}\right) p^{k-1} g_{N} / N$. Three lower bounds on $g_{N}$ were given above. The first ensures that we may apply Theorem 1.2 to bound the probability of the deviation $D^{\mathcal{H}_{N}}\left(B_{m_{-}}\right)>e\left(\mathcal{H}_{N}\right) p^{k-1} g_{N} / N$. The second and third give that the resulting bound is at most $N^{O_{k}(1)} \exp (-\omega(\log N))$ and $N^{O_{k}(1)} \exp \left(-\omega\left(x_{*}^{2}\right)\right)$ respectively. In particular, for any constant $C$ we have that

$$
\mathbb{P}\left(D^{\mathcal{H}_{N}}\left(B_{m_{-}}\right)>e\left(\mathcal{H}_{N}\right) p^{k-1} g_{N} / N\right) \leqslant N^{O_{k}(1)} \exp \left(-C \log N-x_{*}^{2}\right)
$$

for all sufficiently large $N$. Choosing $C$ to be one larger than the constant of the $O_{k}(1)$ we obtain (5-4), and so complete the proof of the lemma. 


\section{6 \\ Deviations in $\mathbf{m}$-model for non-regular hypergraphs}

In this chapter, we prove Theorem 6.1 , which clearly implies Theorem 1.7 stated in the introduction.

Theorem 6.1 Let $\mathcal{H}_{N}$ be a sequence of $k$-uniform hypergraphs such that $V\left(\mathcal{H}_{N}\right)=[N]$ with maximum degree $\Delta=\Delta\left(\mathcal{H}_{N}\right)$ and maximum pair degree $\Delta_{2}\left(\mathcal{H}_{N}\right)=O(1)$ and let $m / N=t \leqslant 1 / 2$. Assume that $\sigma_{d}\left(\mathcal{H}_{N}\right) / \Delta \gg$ $(t \log N / N)^{1 / 4}$ and $\sigma_{d}^{2}\left(\mathcal{H}_{N}\right) / \Delta \gg \log N / t^{k-1}$. Let $a_{N}$ be a sequence such that

$$
\begin{aligned}
& \max \left\{t \Delta, t^{k-1 / 2} \sigma_{d}\left(\mathcal{H}_{N}\right) N^{1 / 2}\right\} \ll a_{N} \ll \\
& \min \left\{\frac{t^{k} \sigma_{d}^{2}\left(\mathcal{H}_{N}\right) N}{\Delta}, \frac{\sigma_{d}^{3}\left(\mathcal{H}_{N}\right) N t^{k-1}}{\Delta^{2}}, \frac{t^{3 k / 2-1} \sigma_{d}^{2}\left(\mathcal{H}_{N}\right) N^{1 / 2}}{\Delta^{1 / 2}}\right\} .
\end{aligned}
$$

Then

$$
\mathbb{P}\left(D^{\mathcal{H}_{N}}\left(B_{m}\right) \geqslant a_{N}\right)=\exp \left(\frac{-(1+o(1)) a_{N}^{2}}{2(1-t) t^{2 k-1} \sigma_{d}^{2}\left(\mathcal{H}_{N}\right) N}\right) .
$$

The proof of Theorem 6.1 is based on an approximation of $D^{\mathcal{H}}\left(B_{m}\right)$ by an expression which only depends on the degrees of the vertices of $B_{m}$. More precisely, we will prove that $D^{\mathcal{H}}\left(B_{m}\right)$ can be well approximated by ${ }^{1}$

$$
\Lambda^{\mathcal{H}, *}\left(B_{m}\right):=\sum_{i=1}^{m} \sum_{\ell=1}^{k} \frac{(1-t)^{\ell}(t-s)^{k-\ell} s^{\ell-1}}{(1-s)^{k}}\left(\begin{array}{c}
k-1 \\
\ell-1
\end{array}\right) X_{1}^{\mathcal{H}}\left(B_{i}\right) .
$$

The conditional variance of this new expression can be well predicted, which will allow us to use Freedman's inequality and its converse. We now give a brief sketch of the proof. We first prove in Section 6.1 that the increments of our martingale representation for $D^{\mathcal{H}}\left(B_{m}\right)$ are well approximated by a linear function of $X_{1}$. This will give rise to a decomposition

$$
D^{\mathcal{H}}\left(B_{m}\right)=\Lambda^{\mathcal{H}}\left(B_{m}\right)+D^{\mathcal{H}, \perp}\left(B_{m}\right),
$$

where $\Lambda^{\mathcal{H}}\left(B_{m}\right)$ is related to $\Lambda^{\mathcal{H}, *}\left(B_{m}\right)$ and $D^{\mathcal{H}, \perp}\left(B_{m}\right)$ is an expression which we may think of as an error term. Relying on the approximation of the increments,

\footnotetext{
${ }^{1}$ We observe that such approach could not work in the regular framework, since in this case the increments $X_{1}$ are equal to 0 .
} 
we control in Section 6.2 the probability that the error term $D^{\mathcal{H}, \perp}\left(B_{m}\right)$ is large. In Section 6.3, we prove that $\Lambda^{\mathcal{H}}\left(B_{m}\right)$ is deterministically well approximated by $\Lambda^{\mathcal{H}, *}\left(B_{m}\right)$. Next, we prove in Section 6.4 that the conditional variance of the increments $X_{1}$ are (with very high probability) close to the variance of the degree sequence of our hypergraph, provided that some conditions on the hypergraph are satisfied. In Section 6.5 , we prove an asymptotic result for the log-probability of deviations of $\Lambda^{\mathcal{H}, *}\left(B_{m}\right)$. Finally we complete the proof of Theorem 6.1 in Section 6.6.

\section{1}

\section{Approximation of the increments}

The proof that the increments $X_{\ell}$ of our martingale representation are well approximated by a function of $X_{1}$ is based on the following result, which is effective only for bounded degree hypergraphs. We observe that this proposition will be applied for the link hypergraph with respect to some vertex, which has bounded maximum degree, since our original hypergraph has bounded maximum pair degree.

Proposition 6.2 Let $m / N=t \leqslant 1 / 2$ and $\mathcal{H}$ be a $k$-uniform hypergraph on $[N]$ with maximum degree $\Delta$. Then, for all $\alpha>0,0 \leqslant j \leqslant k$, and uniformly for all $0 \leqslant i \leqslant m$, we have

$$
\mathbb{P}\left(D_{j}^{\mathcal{H}}\left(B_{i}\right)>\alpha\right) \leqslant N^{O_{k}(1)} \exp \left(-\Omega_{k, \Delta}(1) \min \left\{\alpha, \frac{\alpha^{2}}{e(\mathcal{H}) t^{j}}\right\}\right) .
$$

Proof. We proceed by induction on $j$. Note that for all $0 \leqslant i \leqslant m$ we have $D_{0}^{\mathcal{H}}\left(B_{i}\right)=N_{0}^{\mathcal{H}}\left(B_{i}\right)-\mathbb{E}\left[N_{0}^{\mathcal{H}}\left(B_{i}\right)\right]=0$, since $N_{0}^{\mathcal{H}}\left(B_{i}\right)=e(\mathcal{H})$, the number of edges in $\mathcal{H}$. Thus the base case $j=0$ holds trivially. It is also clear that for $i=0$ we have no deviations and so this case also holds trivially.

Now assume $j \geqslant 1$, fix an arbitrary $\alpha>0$ and we aim to prove the statement for deviations of size $\alpha$ based on it being true for all $0 \leqslant j^{\prime}<j$, deviations $\alpha_{j^{\prime}}>0$, and $0 \leqslant i \leqslant m$. We define

$$
Z_{i}:=\sum_{\ell=1}^{j} \frac{(N-m)_{\ell}(m-i)_{j-\ell}}{(N-i)_{j}}\left(\begin{array}{c}
k-\ell \\
k-j
\end{array}\right) X_{\ell}^{\mathcal{H}}\left(B_{i}\right)
$$

to be the martingale increment of $D_{j}^{\mathcal{H}}\left(B_{m}\right)$ and so

$$
D_{j}^{\mathcal{H}}\left(B_{m}\right)=\sum_{i=1}^{m} Z_{i}
$$


The goal now is to control the conditional variance of this process so we can use Freedman inequality, which will give us the desired bound. Using the CauchySchwarz inequality and

$$
\frac{(N-m)_{\ell}(m-i)_{j-\ell}}{(N-i)_{j}}\left(\begin{array}{l}
k-\ell \\
k-j
\end{array}\right) \leqslant t^{j-\ell} k !,
$$

we have

$$
\mathbb{E}\left[Z_{i}^{2} \mid B_{i-1}\right] \leqslant O_{k}(1) \sum_{\ell=1}^{j} t^{2 j-2 \ell} \mathbb{E}\left[X_{\ell}^{\mathcal{H}}\left(B_{i}\right)^{2} \mid B_{i-1}\right] .
$$

We recall that $X_{\ell}^{\mathcal{H}}\left(B_{i}\right)=A_{\ell}^{\mathcal{H}}\left(B_{i}\right)-\mathbb{E}\left[A_{\ell}^{\mathcal{H}}\left(B_{i}\right) \mid B_{i-1}\right]$, where $A_{\ell}^{\mathcal{H}}\left(B_{i}\right)=$ $N_{\ell}^{\mathcal{H}}\left(B_{i}\right)-N_{\ell}^{\mathcal{H}}\left(B_{i-1}\right)$. Since $\mathbb{E}\left[A_{\ell}^{\mathcal{H}}\left(B_{i}\right) \mid B_{i-1}\right]$ is $B_{i-1}$-measurable, we get

$$
\begin{aligned}
\mathbb{E}\left[X_{\ell}^{\mathcal{H}}\left(B_{i}\right)^{2} \mid B_{i-1}\right] & =\mathbb{E}\left[A_{\ell}^{\mathcal{H}}\left(B_{i}\right)^{2} \mid B_{i-1}\right]-\left(\mathbb{E}\left[A_{\ell}^{\mathcal{H}}\left(B_{i}\right) \mid B_{i-1}\right]\right)^{2} \\
& \leqslant \mathbb{E}\left[A_{\ell}^{\mathcal{H}}\left(B_{i}\right)^{2} \mid B_{i-1}\right]
\end{aligned}
$$

which gives us

$$
\mathbb{E}\left[Z_{i}^{2} \mid B_{i-1}\right] \leqslant O_{k}(1) \sum_{\ell=1}^{j} t^{2 j-2 \ell} \mathbb{E}\left[A_{\ell}^{\mathcal{H}}\left(B_{i}\right)^{2} \mid B_{i-1}\right]
$$

We now fix $1 \leqslant \ell \leqslant j$ for the moment and say that a vertex $x \in[N] \backslash B_{i-1}$ is $\ell$-useful at time $i$ if there are $\ell-1$ distinct vertices $x_{1}, \ldots, x_{\ell-1} \in B_{i-1}$ such that the set $\left\{x, x_{1}, \ldots, x_{\ell-1}\right\}$ is contained in some edge of $\mathcal{H}$. Observe that choosing an element $x \in[N] \backslash B_{i-1}$ which is not $\ell$-useful in step $i$, i.e. $b_{i}=\{x\}$, implies that $N_{\ell}^{\mathcal{H}}\left(B_{i}\right)$ does not increase and so $A_{\ell}^{\mathcal{H}}\left(B_{i}\right)=$ $N_{\ell}^{\mathcal{H}}\left(B_{i}\right)-N_{\ell}^{\mathcal{H}}\left(B_{i-1}\right)=0$. On the other hand, if $B_{i} \backslash B_{i-1}=\{x\}$ and $x$ is $\ell$-useful, there are at most $\Delta$ edges in $\mathcal{H}$ containing $x$ and for each of them there are at most $\left(\begin{array}{c}k-1 \\ \ell-1\end{array}\right)$ ways of completing an $\ell$-subset containing $x$; hence in this case we have $A_{\ell}^{\mathcal{H}}\left(B_{i}\right) \leqslant\left(\begin{array}{c}k-1 \\ \ell-1\end{array}\right) \Delta=O_{k, \Delta}(1)$. We may easily bound the number of $\ell$-useful vertices. Indeed, there are at most $N_{\ell-1}^{\mathcal{H}}\left(B_{i-1}\right)$ edges which contain at least $\ell-1$ vertices of $B_{i-1}$ and each gives us at most $k \ell$-useful vertices. Hence the probability of choosing an $\ell$-useful vertex in step $i$ is at most $k N_{\ell-1}^{\mathcal{H}}\left(B_{i-1}\right) /(N-i+1)$. Altogether, this implies that

$$
\mathbb{E}\left[Z_{i}^{2} \mid B_{i-1}\right] \leqslant O_{k, \Delta}(1) \sum_{\ell=1}^{j} t^{2 j-2 \ell} \frac{N_{\ell-1}^{\mathcal{H}}\left(B_{i-1}\right)}{N-i+1} .
$$

Next we use the induction hypothesis to provide an asymptotically almost sure upper bound on $N_{j^{\prime}-1}^{\mathcal{H}}\left(B_{i}\right)$ that holds uniformly for all $0 \leqslant i \leqslant m$. By the induction hypothesis for $j^{\prime}=\ell-1$ and $\alpha_{\ell-1}=t^{\ell-1-j} \max \left\{\alpha, e(\mathcal{H}) t^{j}\right\}>0$, we 
have

$$
\begin{aligned}
& \mathbb{P}\left(D_{\ell-1}^{\mathcal{H}}\left(B_{i}\right) \leqslant t^{\ell-1-j} \max \left\{\alpha, e(\mathcal{H}) t^{j}\right\}\right) \geqslant \\
& 1-N^{O_{k}(1)} \exp \left(-\Omega_{k, \Delta}(1) \min \left\{\alpha_{\ell-1}, \frac{\alpha_{\ell-1}^{2}}{e(\mathcal{H}) t^{\ell-1}}\right\}\right)
\end{aligned}
$$

uniformly for all $0 \leqslant i \leqslant m$, where we recall that $t=m / N$. We note that

$$
\min \left\{\alpha_{\ell-1}, \frac{\alpha_{\ell-1}^{2}}{e(\mathcal{H}) t^{\ell-1}}\right\}= \begin{cases}\min \left\{t^{\ell-1-j} \alpha, \frac{t^{2(\ell-1-j)} \alpha^{2}}{e(\mathcal{H}) t^{\ell-1}}\right\}=t^{\ell-1-j} \alpha, & \alpha \geqslant e(\mathcal{H}) t^{j} \\ e(\mathcal{H}) t^{\ell-1}, & \alpha<e(\mathcal{H}) t^{j}\end{cases}
$$

and so we obtain

$$
\begin{aligned}
& \mathbb{P}\left(D_{\ell-1}^{\mathcal{H}}\left(B_{i}\right) \leqslant t^{\ell-1-j} \max \left\{\alpha, e(\mathcal{H}) t^{j}\right\}\right)= \\
& 1-N^{O_{k}(1)} \exp \left(-\Omega_{k, \Delta}(1) t^{\ell-1-j} \max \left\{\alpha, e(\mathcal{H}) t^{j}\right\}\right),
\end{aligned}
$$

uniformly for all $0 \leqslant i \leqslant m$.

Thus applying the union bound for $1 \leqslant \ell \leqslant j$ this implies that we have

$$
N_{\ell-1}^{\mathcal{H}}\left(B_{i}\right) \leqslant \mathbb{E}\left[N_{\ell-1}^{\mathcal{H}}\left(B_{i}\right)\right]+\alpha_{\ell-1}
$$

for all $1 \leqslant \ell \leqslant j$ and uniformly for all $0 \leqslant i \leqslant m$, with probability at least

$$
\begin{aligned}
& 1-\sum_{\ell=1}^{j} N^{O_{k}(1)} \exp \left(-\Omega_{k, \Delta}(1) t^{\ell-1-j} \max \left\{\alpha, e(\mathcal{H}) t^{j}\right\}\right) \geqslant \\
& 1-N^{O_{k}(1)} \exp \left(-\Omega_{k, \Delta}(1) \max \left\{\alpha, e(\mathcal{H}) t^{j}\right\}\right) .
\end{aligned}
$$

Furthermore, since by $(3-1)$ we have $\mathbb{E}\left[N_{\ell-1}^{\mathcal{H}}\left(B_{i}\right)\right] \leqslant O_{k}(1) e(\mathcal{H}) t^{\ell-1}$, this implies

$$
\begin{aligned}
N_{\ell-1}^{\mathcal{H}}\left(B_{i}\right) & \leqslant O_{k}(1) e(\mathcal{H}) t^{\ell-1}+\alpha_{\ell-1} \\
& \leqslant O_{k}(1) e(\mathcal{H}) t^{\ell-1}+t^{\ell-1-j} \max \left\{\alpha, e(\mathcal{H}) t^{j}\right\} \\
& \leqslant O_{k}(1) t^{\ell-1-j} \max \left\{\alpha, e(\mathcal{H}) t^{j}\right\},
\end{aligned}
$$

where this bound holds uniformly for all $0 \leqslant i \leqslant m$.

Consequently by (6-1), with probability at least

$$
1-N^{O_{k}(1)} \exp \left(-\Omega_{k, \Delta}(1) \max \left\{\alpha, e(\mathcal{H}) t^{j}\right\}\right)
$$


we can bound the conditional variance of the first $m$ steps by

$$
\begin{aligned}
\sum_{i=0}^{m} \mathbb{E}\left[Z_{i}^{2} \mid B_{i-1}\right] & \leqslant O_{k, \Delta}(1) \max \left\{\alpha, e(\mathcal{H}) t^{j}\right\} \sum_{\ell=1}^{j} t^{j-\ell-1} \sum_{i=1}^{m} \frac{1}{N-i+1} \\
& \leqslant O_{k, \Delta}(1)\left(\alpha+e(\mathcal{H}) t^{j}\right),
\end{aligned}
$$

where $\sum_{i=1}^{m} \frac{1}{N-i+1} \leqslant m /(N-m) \leqslant 2 t$, since $m / N=t \leqslant 1 / 2, \sum_{\ell=1}^{j} t^{j-\ell-1} \leqslant$ $O_{k}(1) t^{-1}$ and we have bounded the maximum by the sum of its terms.

It remains to observe that $\left|Z_{i}\right|=O_{k, \Delta}(1)$, uniformly for all $1 \leqslant i \leqslant m$, and apply Freedman's inequality, Lemma 2.15, (after truncation of the error event) to obtain

$$
\begin{aligned}
& \mathbb{P}\left(D_{j}^{\mathcal{H}}\left(B_{m}\right)>\alpha\right) \leqslant \exp \left(\frac{-\alpha^{2}}{O_{k, \Delta}(1)\left(\alpha+e(\mathcal{H}) t^{j}\right)}\right)+ \\
& N^{O_{k}(1)} \exp \left(-\Omega_{k, \Delta}(1) \max \left\{\alpha, e(\mathcal{H}) t^{j}\right\}\right) \leqslant \\
& N^{O_{k}(1)} \exp \left(-\Omega_{k, \Delta}(1) \min \left\{\alpha, \frac{\alpha^{2}}{e(\mathcal{H}) t^{j}}\right\}\right)
\end{aligned}
$$

as claimed, completing the induction step.

We now prove that $X_{\ell}^{\mathcal{H}}\left(B_{i}\right)$ is well approximated by a linear function of $X_{1}^{\mathcal{H}}\left(B_{i}\right)$. More specifically, we define

$$
Y_{\ell}^{\mathcal{H}}\left(B_{i}\right):=X_{\ell}^{\mathcal{H}}\left(B_{i}\right)-\left(\begin{array}{c}
k-1 \\
\ell-1
\end{array}\right) \frac{(i-1)_{\ell-1}}{(N-1)_{\ell-1}} X_{1}^{\mathcal{H}}\left(B_{i}\right)
$$

and we prove the following proposition.

Proposition 6.3 Let $\mathcal{H}$ be a k-uniform hypergraph on $[N]$ with maximum degree $\Delta$ and maximum pair degree $\Delta_{2}$ and let $t=m / N \leqslant 1 / 2$. Then, for all $0 \leqslant \ell \leqslant k, 0 \leqslant i \leqslant m$, and $\alpha>0$ we have

$$
\mathbb{P}\left(\left|Y_{\ell}^{\mathcal{H}}\left(B_{i}\right)\right|>\alpha\right) \leqslant N^{O_{k}(1)} \exp \left(-\Omega_{k, \Delta_{2}}(1) \min \left\{\frac{\alpha^{2}}{t^{\ell-1} \Delta}, \alpha\right\}\right) .
$$

For the proof it will be convenient to instead consider a quantity similar to $Y_{\ell}^{\mathcal{H}}\left(B_{i}\right)$ obtained by replacing the centralised increments $X_{\ell}^{\mathcal{H}}\left(B_{i}\right)$ by their not centralised counterparts $A_{\ell}^{\mathcal{H}}\left(B_{i}\right)$. In other words, we let

$$
T_{\ell}^{\mathcal{H}}\left(B_{i}\right):=A_{\ell}^{\mathcal{H}}\left(B_{i}\right)-\left(\begin{array}{c}
k-1 \\
\ell-1
\end{array}\right) \frac{(i-1)_{\ell-1}}{(N-1)_{\ell-1}} A_{1}^{\mathcal{H}}\left(B_{i}\right)
$$


and note that we have

$$
Y_{\ell}^{\mathcal{H}}\left(B_{i}\right)=T_{\ell}^{\mathcal{H}}\left(B_{i}\right)-\mathbb{E}\left[T_{\ell}^{\mathcal{H}}\left(B_{i}\right) \mid B_{i-1}\right]
$$

The proof of Proposition 6.3 will be a consequence of the following lemma.

Lemma 6.4 Let $\mathcal{H}$ be a $k$-uniform hypergraph on $[N]$ with maximum degree $\Delta$ and maximum pair degree $\Delta_{2}$ and let $t=m / N \leqslant 1 / 2$. Then, for all $0 \leqslant \ell \leqslant k$, $0 \leqslant i \leqslant m$ and $\alpha \geqslant 0$ we have

$$
\mathbb{P}\left(\left|T_{\ell}^{\mathcal{H}}\left(B_{i}\right)\right|>\alpha\right) \leqslant N^{O_{k}(1)} \exp \left(-\Omega_{k, \Delta_{2}}(1) \min \left\{\frac{\alpha^{2}}{t^{\ell-1} \Delta}, \alpha\right\}\right) .
$$

Proof. We condition on the event $\left\{b_{i}=x\right\}$, i.e., $x$ is added as the $i$-th element of the process and consider the link hypergraph $\mathcal{H}(x)$. Note that given that $b_{i}=x$, the set $B_{i}$ is distributed as $B_{i}=B_{i-1}^{(x)} \cup\{x\}$, where $B_{i-1}^{(x)}$ is a uniformly random subset of $i-1$ elements of $[N] \backslash\{x\}$. Now observe that we have

$$
A_{1}^{\mathcal{H}}\left(B_{i}\right)=\mid\{(x, f): f \in E(\mathcal{H}) \text { and } x \in f\} \mid=d_{\mathcal{H}}(x)=e(\mathcal{H}(x)),
$$

and similarly

$$
\begin{aligned}
A_{\ell}^{\mathcal{H}}\left(B_{i}\right) & =\left|\left\{\left(\left\{x, x_{1}, \ldots, x_{\ell-1}\right\}, f\right): f \in E(\mathcal{H}), x \in f,\left(x_{1}, \ldots, x_{\ell-1}\right) \subseteq B_{i-1}^{(x)} \cap f\right\}\right| \\
& =N_{\ell-1}^{\mathcal{H}(x)}\left(B_{i-1}^{(x)}\right) .
\end{aligned}
$$

Furthermore, note that (3-1) implies

$$
\mathbb{E}\left[N_{\ell-1}^{\mathcal{H}(x)}\left(B_{i-1}^{(x)}\right)\right]=\left(\begin{array}{c}
k-1 \\
\ell-1
\end{array}\right) \frac{(i-1)_{\ell-1}}{(N-1)_{\ell-1}} e(\mathcal{H}(x))
$$

Combining these three statements, we obtain

$$
\begin{aligned}
T_{\ell}^{\mathcal{H}}\left(B_{i}\right) & =A_{\ell}^{\mathcal{H}}\left(B_{i}\right)-\left(\begin{array}{c}
k-1 \\
\ell-1
\end{array}\right) \frac{(i-1)_{\ell-1}}{(N-1)_{\ell-1}} A_{1}^{\mathcal{H}}\left(B_{i}\right) \\
& =N_{\ell-1}^{\mathcal{H}(x)}\left(B_{i-1}^{(x)}\right)-\mathbb{E}\left[N_{\ell-1}^{\mathcal{H}(x)}\left(B_{i-1}^{(x)}\right)\right] \\
& =D_{\ell-1}^{\mathcal{H}(x)}\left(B_{i-1}^{(x)}\right)
\end{aligned}
$$

and so Proposition 6.2 implies

$$
\mathbb{P}\left(\left|D_{\ell-1}^{\mathcal{H}(x)}\left(B_{i-1}^{(x)}\right)\right|>\alpha\right) \leqslant N^{O_{k}(1)} \exp \left(-\Omega_{k, \Delta(\mathcal{H}(x))}(1) \min \left\{\frac{\alpha^{2}}{e(\mathcal{H}(x)) t^{\ell-1}}, \alpha\right\}\right) .
$$


Since $e(\mathcal{H}(x))=d_{\mathcal{H}}(x) \leqslant \Delta_{\mathcal{H}}$ and $\Delta_{\mathcal{H}(x)} \leqslant \Delta_{2}(\mathcal{H})$ we obtain

$$
\mathbb{P}\left(\left|T_{\ell}^{\mathcal{H}}\left(B_{i}\right)\right|>\alpha \mid b_{i}=x\right) \leqslant N^{O_{k}(1)} \exp \left(-\Omega_{k, \Delta_{2}}(1) \min \left\{\frac{\alpha^{2}}{t^{\ell-1} \Delta}, \alpha\right\}\right)
$$

uniformly for all $x \in[N] \backslash B_{i-1}$, hence also unconditionally.

We now show that Proposition 6.3 follows from Lemma 6.4

Proof of Proposition 6.3. Recall that $Y_{\ell}^{\mathcal{H}}\left(B_{i}\right)=T_{\ell}^{\mathcal{H}}\left(B_{i}\right)-\mathbb{E}\left[T_{\ell}^{\mathcal{H}}\left(B_{i}\right) \mid B_{i-1}\right]$. Hence, by the triangle inequality, the event $\left|Y_{\ell}^{\mathcal{H}}\left(B_{i}\right)\right|>\alpha$ may only occur if either $\left|T_{\ell}^{\mathcal{H}}\left(B_{i}\right)\right|>\alpha / 2$ or $\left|\mathbb{E}\left[T_{\ell}^{\mathcal{H}}\left(B_{i}\right) \mid B_{i-1}\right]\right|>\alpha / 2$. By Lemma 6.4 , the first event has probability

$$
\mathbb{P}\left(\left|T_{\ell}^{\mathcal{H}}\left(B_{i}\right)\right|>\alpha / 2\right) \leqslant N^{O_{k}(1)} \exp \left(-\Omega_{k, \Delta_{2}}(1) \min \left\{\frac{\alpha^{2}}{t^{\ell-1} \Delta}, \alpha\right\}\right) .
$$

On the other hand, the second event may only occur if there exists an extension $x \in[N] \backslash B_{i-1}$ such that $\left|T_{\ell}^{\mathcal{H}}\left(B_{i}\right)\right|>\alpha / 2$ conditionally on $b_{i}=x$. So by Lemma 3.2 we have

$$
\mathbb{P}\left(\left|\mathbb{E}\left[T_{\ell}^{\mathcal{H}}\left(B_{i}\right) \mid B_{i-1}\right]\right|>\alpha / 2\right) \leqslant N \mathbb{P}\left(\left|T_{\ell}^{\mathcal{H}}\left(B_{i}\right)\right|>\alpha / 2\right)
$$

completing the proof.

\section{2}

\section{Control of the error term}

We first recall that we defined

$$
Y_{\ell}^{\mathcal{H}}\left(B_{i}\right):=X_{\ell}^{\mathcal{H}}\left(B_{i}\right)-\left(\begin{array}{c}
k-1 \\
\ell-1
\end{array}\right) \frac{(i-1)_{\ell-1}}{(N-1)_{\ell-1}} X_{1}^{\mathcal{H}}\left(B_{i}\right)
$$

and that Lemma 3.1 gives us

$$
D^{\mathcal{H}}\left(B_{m}\right)=\sum_{i=1}^{m} \sum_{\ell=1}^{k} \frac{(N-m)_{\ell}(m-i)_{k-\ell}}{(N-i)_{k}} X_{\ell}^{\mathcal{H}}\left(B_{i}\right) .
$$

Therefore we can write

$$
D^{\mathcal{H}}\left(B_{m}\right)=\Lambda^{\mathcal{H}}\left(B_{m}\right)+D^{\mathcal{H}, \perp}\left(B_{m}\right)
$$

where

$$
\Lambda^{\mathcal{H}}\left(B_{m}\right)=\sum_{i=1}^{m} \sum_{\ell=1}^{k} \frac{(N-m)_{\ell}(m-i)_{k-\ell}}{(N-i)_{k}}\left(\begin{array}{c}
k-1 \\
\ell-1
\end{array}\right) \frac{(i-1)_{\ell-1}}{(N-1)_{\ell-1}} X_{1}^{\mathcal{H}}\left(B_{i}\right)
$$


is the main part of the deviation and

$$
D^{\mathcal{H}, \perp}\left(B_{m}\right)=\sum_{i=1}^{m} \sum_{\ell=1}^{k} \frac{(N-m)_{\ell}(m-i)_{k-\ell}}{(N-i)_{k}} Y_{\ell}^{\mathcal{H}}\left(B_{i}\right)
$$

expresses $D^{\mathcal{H}, \perp}\left(B_{m}\right)$ as the final value of a martingale whose increments are weighted sums of the $Y_{\ell}^{\mathcal{H}}\left(B_{i}\right)$. We control the probability that $D^{\mathcal{H}, \perp}\left(B_{m}\right)$ is large using Lemma 3.3 (a variant of Hoeffding-Azuma), together with Proposition 6.3, which helps us control the size of $\left|Y_{\ell}^{\mathcal{H}}\left(B_{i}\right)\right|$, and therefore the increments of the martingale. Set

$$
\mathbb{Y}_{i}:=\sum_{\ell=1}^{k} \frac{(N-m)_{\ell}(m-i)_{k-\ell}}{(N-i)_{k}} Y_{\ell}^{\mathcal{H}}\left(B_{i}\right)
$$

Proposition 6.5 Let $\mathcal{H}$ be a k-uniform hypergraph on $[N]$ with maximum degree $\Delta$ and maximum pair degree $\Delta_{2}$ and let $t=m / N \leqslant 1 / 2$. Then for all $\theta \geqslant 1$ and $\alpha>0$, we have

$\mathbb{P}\left(D^{\mathcal{H}, \perp}\left(B_{m}\right)>\alpha\right) \leqslant \exp \left(\frac{-\theta \alpha^{2}}{\sigma_{d}^{2}(\mathcal{H}) t^{2 k-1} N}\right)+N^{O_{k}(1)} \exp \left(\frac{-\Omega_{k, \Delta_{2}}(1) t^{k-1} \sigma_{d}^{2}(\mathcal{H})}{\theta \Delta}\right)$

Furthermore, the same bound holds for $\mathbb{P}\left(D^{\mathcal{H}, \perp}\left(B_{m}\right)<-\alpha\right)$.

Proof. Since we have a martingale expression $D^{\mathcal{H}, \perp}\left(B_{m}\right)=\sum_{i=1}^{m} \mathbb{Y}_{i}$ for $D^{\mathcal{H}, \perp}\left(B_{m}\right)$ the required result follows immediately from the variant of Hoeffding-Azuma inequality (Lemma 3.3) and the bound

$$
\mathbb{P}\left(\left|\mathbb{Y}_{i}\right| \geqslant \theta^{-1 / 2} \sigma_{d}(\mathcal{H}) t^{k-1}\right) \leqslant N^{O_{k}(1)} \exp \left(\frac{-\Omega_{k, \Delta_{2}}(1) t^{k-1} \sigma_{d}^{2}(\mathcal{H})}{\theta \Delta}\right)
$$

for all $1 \leqslant i \leqslant m$.

We shall prove (6-3) by applying Proposition 6.3 to the $Y_{\ell}^{\mathcal{H}}\left(B_{i}\right)$. Considering the definition of $\mathbb{Y}_{i}$ and the fact that the coefficient of $Y_{\ell}^{\mathcal{H}}\left(B_{i}\right)$ is at most $t^{k-\ell}$, by the triangle inequality and the union bound, it suffices to prove that

$$
\mathbb{P}\left(\left|Y_{\ell}^{\mathcal{H}}\left(B_{i}\right)\right| \geqslant \frac{\sigma_{d}(\mathcal{H}) t^{\ell-1}}{k \theta^{1 / 2}}\right) \leqslant N^{O_{k}(1)}\left(\frac{-\Omega_{k, \Delta_{2}}(1) t^{k-1} \sigma_{d}^{2}(\mathcal{H})}{\theta \Delta}\right)
$$

for all $\ell=1, \ldots, k$. Recall that the conclusion of Proposition 6.3 contains a minimum. Since $\sigma_{d}(\mathcal{H}) t^{\ell-1} / k \theta^{1 / 2} \leqslant \Delta t^{\ell-1}$ the minimum is achieved by the quadratic term. It follows that

$$
\mathbb{P}\left(\left|Y_{\ell}^{\mathcal{H}}\left(B_{i}\right)\right| \geqslant \frac{\sigma_{d}(\mathcal{H}) t^{\ell-1}}{k \theta^{1 / 2}}\right) \leqslant N^{O_{k}(1)} \exp \left(\frac{-\Omega_{k, \Delta_{2}}(1) t^{\ell-1} \sigma_{d}^{2}(\mathcal{H})}{\theta \Delta}\right) .
$$

Recalling that $\ell \leqslant k$, this gives the required bound (6-4). 


\section{3}

\section{Approximation of $\Lambda^{\mathcal{H}}\left(B_{m}\right)$}

Recall that we have defined the following expression in the previous section

$$
\Lambda^{\mathcal{H}}\left(B_{m}\right)=\sum_{i=1}^{m} \sum_{\ell=1}^{k} \frac{(N-m)_{\ell}(m-i)_{k-\ell}}{(N-i)_{k}}\left(\begin{array}{c}
k-1 \\
\ell-1
\end{array}\right) \frac{(i-1)_{\ell-1}}{(N-1)_{\ell-1}} X_{1}^{\mathcal{H}}\left(B_{i}\right) .
$$

Besides this expression, we consider the following approximation of $\Lambda^{\mathcal{H}}\left(B_{m}\right)$, which we have defined in the beginning of this chapter

$$
\Lambda^{\mathcal{H}, *}\left(B_{m}\right)=\sum_{i=1}^{m} \sum_{\ell=1}^{k} \frac{(1-t)^{\ell}(t-s)^{k-\ell} s^{\ell-1}}{(1-s)^{k}}\left(\begin{array}{c}
k-1 \\
\ell-1
\end{array}\right) X_{1}^{\mathcal{H}}\left(B_{i}\right) .
$$

This expression can be simplified by an immediate application of the binomial theorem. We remark that the first expression we give for $\Lambda^{\mathcal{H}, *}\left(B_{m}\right)$ will be used in this section, whereas the second, given by (6-5), will be employed in Sections 6.4 and 6.5 .

Lemma 6.6 Given real numbers $s, t$, with $s \neq 1$ and a positive integer $k$, the following identity holds

$$
\sum_{\ell=1}^{k} \frac{(1-t)^{\ell}(t-s)^{k-\ell} s^{\ell-1}}{(1-s)^{k}}\left(\begin{array}{c}
k-1 \\
\ell-1
\end{array}\right)=\frac{t^{k-1}(1-t)}{1-s}
$$

Proof. We first rewrite the sum as

$$
\frac{(1-t)}{(1-s)^{k}} \sum_{\ell=1}^{k}(s-s t)^{\ell-1}(t-s)^{k-\ell}\left(\begin{array}{c}
k-1 \\
\ell-1
\end{array}\right) .
$$

By the binomial theorem, this last sum is equal to

$$
(s-s t+t-s)^{k-1}=t^{k-1}(1-s)^{k-1}
$$

and therefore we obtain the required identity.

By Lemma 6.6, recalling that $s=i / N$, we obtain the following formula.

$$
\Lambda^{\mathcal{H}, *}\left(B_{m}\right)=(1-t) t^{k-1} \sum_{i=1}^{m} \frac{N}{N-i} X_{1}^{\mathcal{H}}\left(B_{i}\right) .
$$

We now prove that $\Lambda^{\mathcal{H}, *}\left(B_{m}\right)$ is indeed a good approximation for $\Lambda^{\mathcal{H}}\left(B_{m}\right)$, which is made precise by the following lemma. 
Lemma 6.7 Let $\mathcal{H}$ be a $k$-uniform hypergraph on $[N]$ with maximum degree $\Delta$ and let $t=m / N \leqslant 1 / 2$. There is a constant $C=C(k)$ such that

$$
\left|\Lambda^{\mathcal{H}}\left(B_{m}\right)-\Lambda^{\mathcal{H}, *}\left(B_{m}\right)\right| \leqslant C t \Delta
$$

Proof. We first observe that the above difference is equal to

$\sum_{i=1}^{m} \sum_{\ell=1}^{k}\left[\frac{(N-m)_{\ell}(m-i)_{k-\ell}(i-1)_{\ell-1}}{(N-i)_{k}(N-1)_{\ell-1}}-\frac{(1-t)^{\ell}(t-s)^{k-\ell} s^{\ell-1}}{(1-s)^{k}}\right]\left(\begin{array}{c}k-1 \\ \ell-1\end{array}\right) X_{1}^{\mathcal{H}}\left(B_{i}\right)$.

Since the outer sum consists of $m=t N$ terms, the inner sum consists of $k$ terms, $\left(\begin{array}{c}k-1 \\ \ell-1\end{array}\right)=O_{k}(1)$, and $\left|X_{1}^{\mathcal{H}}\left(B_{i}\right)\right|=O_{k}(\Delta)$ for each $1 \leqslant i \leqslant m$, it is sufficient to prove the following claim.

Claim There exists a constant $C^{\prime}=C^{\prime}(k)$ such that for all $1 \leqslant i \leqslant m$,

$$
\left|\frac{(N-m)_{\ell}(m-i)_{k-\ell}(i-1)_{\ell-1}}{(N-i)_{k}(N-1)_{\ell-1}}-\frac{(1-t)^{\ell}(t-s)^{k-\ell} s^{\ell-1}}{(1-s)^{k}}\right| \leqslant \frac{C^{\prime}}{N} .
$$

Proof of Claim. We first observe that we can assume that $N$ is sufficiently large, because otherwise we could adjust the constant so the result holds for small values of $N$. Set $x=N-i, y=m-i$ and so $x-y=N-m$. Hence the difference inside the absolute value is equal to

$$
\frac{(x-y)_{\ell}(y)_{k-\ell}(i-1)_{\ell-1}}{(x)_{k}(N-1)_{\ell-1}}-\frac{(x-y)^{\ell} y^{k-\ell} i^{\ell-1}}{x^{k} N^{\ell-1}} .
$$

This expression may be rewritten as

$$
\frac{(x-y)_{\ell}(y)_{k-\ell}(i-1)_{\ell-1} N^{\ell-1} x^{k-1}-(x-y)^{\ell} y^{k-\ell} i^{\ell-1}(N-1)_{\ell-1}(x-1)_{k-1}}{x^{k}(x-1)_{k-1} N^{\ell-1}(N-1)_{\ell-1}} .
$$

We now observe that the numerator of this fraction can be reorganized as the sum of the following five terms

$$
\begin{aligned}
& {\left[(x-y)_{\ell}-(x-y)^{\ell}\right](y)_{k-\ell}(i-1)_{\ell-1} N^{\ell-1} x^{k-1},} \\
& {\left[(y)_{k-\ell}-y^{k-\ell}\right](x-y)^{\ell}(i-1)_{\ell-1} N^{\ell-1} x^{k-1},} \\
& {\left[(i-1)_{\ell-1}-i^{\ell-1}\right](x-y)^{\ell} y^{k-\ell} N^{\ell-1} x^{k-1},} \\
& {\left[N^{\ell-1}-(N-1)_{\ell-1}\right](x-y)^{\ell} y^{k-\ell} i^{\ell-1} x^{k-1},} \\
& {\left[x^{k-1}-(x-1)_{k-1}\right](x-y)^{\ell} y^{k-\ell} i^{\ell-1}(N-1)_{\ell-1} .}
\end{aligned}
$$


Since $y \leqslant x$, the highest order term cancels inside each of the brackets and since $\ell \leqslant k$ and $x-y \leqslant N, y \leqslant N, i \leqslant N, x \leqslant N$, by the triangle inequality there is a constant $C_{1}=C_{1}(k)$ such that the numerator has absolute value at most

$$
C_{1} N^{2 k+2 \ell-4}
$$

Finally, for sufficiently large $N$, we have

$$
x^{k}(x-1)_{k-1} N^{\ell-1}(N-1)_{\ell-1} \geqslant \frac{1}{2} x^{2 k-1} N^{2 \ell-2} .
$$

Therefore

$$
\left|\frac{(N-m)_{\ell}(m-i)_{k-\ell}(i-1)_{\ell-1}}{(N-i)_{k}(N-1)_{\ell-1}}-\frac{(1-t)^{\ell}(t-s)^{k-\ell} s^{\ell-1}}{(1-s)^{k}}\right| \leqslant \frac{2 C_{1} N^{2 k-2}}{x^{2 k-1}} .
$$

Since $x=N-i \geqslant N / 2$, we obtain the desired result with $C^{\prime}=4^{k} C_{1}$.

This concludes the proof of the lemma.

\section{4}

\section{Conditional variance of $\Lambda^{\mathcal{H}, *}\left(B_{m}\right)$}

Recalling the formula obtained for $\Lambda^{\mathcal{H}, *}\left(B_{m}\right)$ obtained in (6-5), we observe that the conditional expectation $\mathbb{E}\left[X_{1}^{\mathcal{H}}\left(B_{i}\right)^{2} \mid B_{i-1}\right]$ plays a key role in the conditional variance of the process. Since $X_{1}^{\mathcal{H}}\left(B_{i}\right)=A_{1}^{\mathcal{H}}\left(B_{i}\right)-\mathbb{E}\left[A_{1}^{\mathcal{H}}\left(B_{i}\right) \mid B_{i-1}\right]$, it follows that

$$
\mathbb{E}\left[X_{1}^{\mathcal{H}}\left(B_{i}\right)^{2} \mid B_{i-1}\right]=\mathbb{E}\left[A_{1}^{\mathcal{H}}\left(B_{i}\right)^{2} \mid B_{i-1}\right]-\left(\mathbb{E}\left[A_{1}^{\mathcal{H}}\left(B_{i}\right) \mid B_{i-1}\right]\right)^{2}
$$

and so if we understand both the quantities on the right hand side of the above equality, we may be able to understand the desired conditional expectation. Furthermore we note that since $A_{1}^{\mathcal{H}}\left(B_{i}\right)=N_{1}^{\mathcal{H}}\left(B_{i}\right)-N_{1}^{\mathcal{H}}\left(B_{i-1}\right)=d_{\mathcal{H}}\left(b_{i}\right)$, where we recall that $b_{i}$ is the $i$-th element added in the process, we have

$$
\mathbb{E}\left[A_{1}^{\mathcal{H}}\left(B_{i}\right)^{2} \mid B_{i-1}\right]=\frac{1}{N-i+1} \sum_{x \in[N] \backslash B_{i-1}} d_{\mathcal{H}}(x)^{2}
$$

and

$$
\mathbb{E}\left[A_{1}^{\mathcal{H}}\left(B_{i}\right) \mid B_{i-1}\right]=\frac{1}{N-i+1} \sum_{x \in[N] \backslash B_{i-1}} d_{\mathcal{H}}(x) .
$$

Motivated by the above expressions, we will obtain results in a more general framework. Namely, given a function $f:[N] \rightarrow \mathbb{R}_{\geqslant 0}{ }^{2}$, we denote by

${ }^{2} \mathbb{R}_{\geqslant 0}$ denotes the set of the nonnegative real numbers. 
$\Delta_{f}:=\max _{x \in[N]} f(x)$ the maximum value of $f$ and we set

$$
\bar{f}=\frac{1}{N} \sum_{x \in[N]} f(x)
$$

the average value of $f$. Moreover, for a set $B \subset[N]$ we define

$$
N_{f}(B)=\sum_{x \in B} f(x)
$$

and

$$
D_{f}(B)=N_{f}(B)-\bar{f}|B|
$$

Besides that, we define the increase in $N_{f}\left(B_{i}\right)$ when the $i$-th element is added by

$$
A_{f}\left(B_{i}\right)=N_{f}\left(B_{i}\right)-N_{f}\left(B_{i-1}\right)
$$

and we define the martingale increments as

$$
X_{f}\left(B_{i}\right)=A_{f}\left(B_{i}\right)-\mathbb{E}\left[A_{f}\left(B_{i}\right) \mid B_{i-1}\right] .
$$

We have the following martingale representation for $D_{f}\left(B_{m}\right)$, in the same spirit as Lemma 3.1. Although the proof of the lemma is very similar to the proof of Lemma 3.1, we include it here for the sake of completeness.

Lemma 6.8 Let $\mathcal{H}$ be a hypergraph on $[N]$ and a function $f:[N] \rightarrow \mathbb{R}_{\geqslant 0}$. For all $0 \leqslant m \leqslant N$, we have

$$
D_{f}\left(B_{m}\right)=\sum_{i=1}^{m} \frac{(N-m)}{(N-i)} X_{f}\left(B_{i}\right) .
$$

Proof. The proof is by induction on $m$. For $m=0$, both sides of the above expression are equal to 0 and thus the base case holds. Assume that the result is true for $m^{\prime}<m$. Since $A_{f}\left(B_{m}\right)=N_{f}\left(B_{m}\right)-N_{f}\left(B_{m-1}\right)=f\left(b_{m}\right)$ and each vertex has probability $1 /(N-m+1)$ to be selected next, we have

$$
\begin{aligned}
\mathbb{E}\left[A_{f}\left(B_{m}\right) \mid B_{m-1}\right] & =\frac{1}{N-m+1} \sum_{x \in[N] \backslash B_{m-1}} f(x) \\
& =\frac{\bar{f} N-N_{f}\left(B_{m-1}\right)}{N-m+1} \\
& =\bar{f}-\frac{D_{f}\left(B_{m-1}\right)}{N-m+1}
\end{aligned}
$$

where in the last step we used that $N_{f}\left(B_{m-1}\right)=D_{f}\left(B_{m-1}\right)+(m-1) \bar{f}$, since $\left|B_{m-1}\right|=m-1$. Recalling that $\mathbb{E}\left[A_{f}\left(B_{m}\right) \mid B_{m-1}\right]=A_{f}\left(B_{m}\right)-X_{f}\left(B_{m}\right)$ and 
replacing

$$
\begin{aligned}
A_{f}\left(B_{m}\right) & =N_{f}\left(B_{m}\right)-N_{f}\left(B_{m-1}\right) \\
& =D_{f}\left(B_{m}\right)-D_{f}\left(B_{m-1}\right)+\bar{f}
\end{aligned}
$$

we obtain

$$
D_{f}\left(B_{m}\right)=\frac{(N-m)}{(N-m+1)} D_{f}\left(B_{m-1}\right)+X_{f}\left(B_{m}\right) .
$$

The required expression (6-6) now follows immediately from the induction hypothesis by checking the coefficient of each $X_{f}\left(B_{i}\right)$.

Based on the above martingale representation for $D_{f}\left(B_{m}\right)$, we now prove the following proposition, which is a simple application of the Hoeffding-Azuma inequality.

Proposition 6.9 Let $\mathcal{H}$ be a k-uniform hypergraph on $[N]$ and a function $f:[N] \rightarrow \mathbb{R}_{\geqslant 0}$. Then, for all $\alpha>0$ and $0 \leqslant m \leqslant N$, we have

$$
\mathbb{P}\left(D_{f}\left(B_{m}\right)>\alpha\right) \leqslant \exp \left(\frac{-\alpha^{2}}{m \Delta_{f}^{2}}\right) .
$$

Furthermore, the same bound applies to the corresponding negative deviations and therefore

$$
\mathbb{P}\left(\left|D_{f}\left(B_{m}\right)\right|>\alpha\right) \leqslant 2 \exp \left(\frac{-\alpha^{2}}{m \Delta_{f}^{2}}\right)
$$

Proof. In the expression obtained in (6-6), we first observe that the coefficients $(N-m) /(N-i)$ are at most 1 . Recalling that $X_{f}\left(B_{i}\right)=A_{f}\left(B_{i}\right)-$ $\mathbb{E}\left[A_{f}\left(B_{i}\right) \mid B_{i-1}\right]$ and that both $A_{f}\left(B_{i}\right)$ and $\mathbb{E}\left[A_{f}\left(B_{i}\right) \mid B_{i-1}\right]$ are non-negative and at most $\Delta_{f}$ ( since $\left.A_{f}\left(B_{i}\right)=f\left(b_{i}\right) \leqslant \Delta_{f}\right)$, we have

$$
\left\|X_{f}\left(B_{i}\right)\right\|_{\infty} \leqslant \Delta_{f} \quad \text { a.s. }
$$

Therefore by an application of Hoeffding-Azuma inequality, we obtain

$$
\mathbb{P}\left(D_{f}\left(B_{m}\right)>\alpha\right) \leqslant \exp \left(\frac{-\alpha^{2}}{m \Delta_{f}^{2}}\right)
$$

as desired. The result for negative deviations follows in the same way.

Now we are in position to prove a result that controls the difference between $\mathbb{E}\left[X_{1}^{\mathcal{H}}\left(B_{i}\right)^{2} \mid B_{i-1}\right]$ and $\sigma_{d}^{2}(\mathcal{H})$, the variance of the degree sequence of $\mathcal{H}$. 
Proposition 6.10 Let $\mathcal{H}$ be a k-uniform hypergraph on $[N]$ with maximum degree $\Delta$ and let $m \leqslant N / 2$. For all $\alpha>0$ and $1 \leqslant i \leqslant m$, we have

$$
\mathbb{P}\left(\left|\mathbb{E}\left[X_{1}^{\mathcal{H}}\left(B_{i}\right)^{2} \mid B_{i-1}\right]-\sigma_{d}^{2}(\mathcal{H})\right|>\alpha\right) \leqslant 4 \exp \left(-\frac{\alpha^{2} N^{2}}{64 m \Delta^{4}}\right) .
$$

Proof. For each $x \in[N]$, we denote $f_{1}(x)=d_{\mathcal{H}}(x)$ and $f_{2}(x)=d_{\mathcal{H}}(x)^{2}$. Observe that

$$
\begin{aligned}
\mathbb{E}\left[A_{1}^{\mathcal{H}}\left(B_{i}\right)^{2} \mid B_{i-1}\right] & =\frac{1}{N-i+1} \sum_{x \in[N] \backslash B_{i-1}} d_{\mathcal{H}}(x)^{2} \\
& =\bar{f}_{2}-\frac{D_{f_{2}}\left(B_{i-1}\right)}{N-i+1}
\end{aligned}
$$

and

$$
\begin{aligned}
\mathbb{E}\left[A_{1}^{\mathcal{H}}\left(B_{i}\right) \mid B_{i-1}\right] & =\frac{1}{N-i+1} \sum_{x \in[N] \backslash B_{i-1}} d_{\mathcal{H}}(x) \\
& =\bar{f}_{1}-\frac{D_{f_{1}}\left(B_{i-1}\right)}{N-i+1}
\end{aligned}
$$

We now prove the following claims.

\section{Claim}

$$
\mathbb{P}\left(\left|\mathbb{E}\left[A_{1}^{\mathcal{H}}\left(B_{i}\right)^{2} \mid B_{i-1}\right]-\bar{f}_{2}\right|>\alpha / 2\right) \leqslant 2 \exp \left(-\frac{\alpha^{2} N^{2}}{16 m \Delta^{4}}\right)
$$

Proof of Claim. We first observe that

$$
\left|\mathbb{E}\left[A_{1}^{\mathcal{H}}\left(B_{i}\right)^{2} \mid B_{i-1}\right]-\bar{f}_{2}\right|=\left|\frac{D_{f_{2}}\left(B_{i-1}\right)}{N-i+1}\right|
$$

Since $N-i+1 \geqslant N / 2, i \leqslant m$ and $\Delta_{f_{2}}=\Delta^{2}$, Proposition 6.9 establishes the claim.

\section{Claim}

$$
\mathbb{P}\left(\left|\left(\mathbb{E}\left[A_{1}^{\mathcal{H}}\left(B_{i}\right) \mid B_{i-1}\right]\right)^{2}-\bar{f}_{1}^{2}\right|>\alpha / 2\right) \leqslant 2 \exp \left(-\frac{\alpha^{2} N^{2}}{64 m \Delta^{4}}\right) .
$$

Proof of Claim. We first observe that

$$
\left|\mathbb{E}\left[A_{1}^{\mathcal{H}}\left(B_{i}\right) \mid B_{i-1}\right]-\bar{f}_{1}\right|=\left|\frac{D_{f_{1}}\left(B_{i-1}\right)}{N-i+1}\right|
$$


and

$$
\left|\mathbb{E}\left[A_{1}^{\mathcal{H}}\left(B_{i}\right) \mid B_{i-1}\right]+\bar{f}_{1}\right| \leqslant 2 \Delta .
$$

Since $N-i+1 \geqslant N / 2, i \leqslant m$ and $\Delta_{f_{1}}=\Delta$, Proposition 6.9 establishes the claim.

To finish the proof of the proposition, we note that

$$
\mathbb{E}\left[X_{1}^{\mathcal{H}}\left(B_{i}\right)^{2} \mid B_{i-1}\right]=\mathbb{E}\left[A_{1}^{\mathcal{H}}\left(B_{i}\right)^{2} \mid B_{i-1}\right]-\left(\mathbb{E}\left[A_{1}^{\mathcal{H}}\left(B_{i}\right) \mid B_{i-1}\right]\right)^{2}
$$

and $\sigma_{d}^{2}(\mathcal{H})=\bar{f}_{2}-\bar{f}_{1}^{2}$. Thus the above two claims and the triangle inequality give us the desired result.

\section{5}

\section{Deviations for $\Lambda^{\mathcal{H}, *}\left(B_{m}\right)$}

Here we prove the following result, which provides the asymptotic of the log-probability of deviations of $\Lambda^{\mathcal{H}_{N}, *}\left(B_{m}\right)$. The proof makes use of Proposition 6.10 to control the conditional variance of the process and Freedman's inequalities (Lemmas 2.15 and 2.16).

Proposition 6.11 Let $\mathcal{H}_{N}$ be a sequence of k-uniform hypergraphs such that $V\left(\mathcal{H}_{N}\right)=[N]$ with maximum degree $\Delta=\Delta\left(\mathcal{H}_{N}\right)$ and let $m / N=t \leqslant 1 / 2$. Assume that $\sigma_{d}\left(\mathcal{H}_{N}\right) / \Delta \gg(t \log N / N)^{1 / 4}$ and let $a_{N}$ be a sequence such that

$$
t^{k-1 / 2} \sigma_{d}\left(\mathcal{H}_{N}\right) N^{1 / 2} \ll a_{N} \ll \min \left\{\frac{t^{k} \sigma_{d}^{2}\left(\mathcal{H}_{N}\right) N}{\Delta}, \frac{\sigma_{d}^{3}\left(\mathcal{H}_{N}\right) N t^{k-1}}{\Delta^{2}}\right\} .
$$

Then

$$
\mathbb{P}\left(\Lambda^{\mathcal{H}_{N}, *}\left(B_{m}\right) \geqslant a_{N}\right)=\exp \left(\frac{-(1+o(1)) a_{N}^{2}}{2(1-t) t^{2 k-1} \sigma_{d}^{2}\left(\mathcal{H}_{N}\right) N}\right)
$$

Proof. We start by recalling the expression for $\Lambda^{\mathcal{H}_{N}, *}\left(B_{m}\right)$, obtained in (6-5).

$$
\Lambda^{\mathcal{H}_{N}, *}\left(B_{m}\right)=(1-t) t^{k-1} \sum_{i=1}^{m} \frac{N}{N-i} X_{1}^{\mathcal{H}_{N}}\left(B_{i}\right) .
$$

We first observe that the event that $\Lambda^{\mathcal{H}_{N}, *}\left(B_{m}\right) \geqslant a_{N}$ is equivalent to

$$
S_{m} \geqslant \frac{a_{N}}{t^{k-1}(1-t)}
$$

where $S_{m}:=\sum_{i=1}^{m} \frac{N}{N-i} X_{1}^{\mathcal{H}_{N}}\left(B_{i}\right)$ and so it is sufficient to prove that

$$
\mathbb{P}\left(S_{m} \geqslant \frac{a_{N}}{t^{k-1}(1-t)}\right)=\exp \left(\frac{-(1+o(1)) a_{N}^{2}}{2(1-t) t^{2 k-1} \sigma_{d}^{2}\left(\mathcal{H}_{N}\right) N}\right) .
$$


We will split the proof of (6-7) into two parts, an upper bound and a lower bound. The upper bound will be based on Freedman's inequality while the lower bound on its converse, which requires controlling the following quantity

$$
V(m):=\sum_{i=1}^{m} \frac{N^{2}}{(N-i)^{2}} \mathbb{E}\left[X_{1}^{\mathcal{H}_{N}}\left(B_{i}\right)^{2} \mid B_{i-1}\right]
$$

Fixing $0<\varepsilon<1$, for all $1 \leqslant i \leqslant m$, Proposition 6.10 gives us

$$
\mathbb{P}\left(\left|\mathbb{E}\left[X_{1}^{\mathcal{H}_{N}}\left(B_{i}\right)^{2} \mid B_{i-1}\right]-\sigma_{d}^{2}\left(\mathcal{H}_{N}\right)\right|>\frac{\varepsilon}{2} \sigma_{d}^{2}\left(\mathcal{H}_{N}\right)\right) \leqslant 4 \exp \left(-\frac{\varepsilon^{2} \sigma_{d}^{4}\left(\mathcal{H}_{N}\right) N^{2}}{256 m \Delta^{4}}\right)
$$

Furthermore, by Riemann sum, we have

$$
\begin{aligned}
\sum_{i=1}^{m} \frac{N^{2}}{(N-i)^{2}} & =N(1+o(1)) \int_{0}^{t} \frac{1}{(1-s)^{2}} d s \\
& =(1+o(1)) \frac{N t}{1-t},
\end{aligned}
$$

provided that $1 / N \ll t \leqslant 1 / 2$ (note that the constraint $1 / N \ll t$ follows from the conditions imposed on $a_{N}$, otherwise the interval of possible $a_{N}$ would be empty). Therefore for all sufficiently large $N$, it follows that

$$
(1-\varepsilon / 3) \frac{N t}{1-t} \leqslant \sum_{i=1}^{m} \frac{N^{2}}{(N-i)^{2}} \leqslant(1+\varepsilon / 3) \frac{N t}{1-t} .
$$

Altogether, defining $E_{N}^{m}(\varepsilon)$ as the event that

$$
(1-\varepsilon) \sigma_{d}^{2}\left(\mathcal{H}_{N}\right) \frac{N t}{1-t} \leqslant V(m) \leqslant(1+\varepsilon) \sigma_{d}^{2}\left(\mathcal{H}_{N}\right) \frac{N t}{1-t},
$$

we obtain that, for all sufficiently large N,

$$
\begin{aligned}
\mathbb{P}\left(E_{N}^{m}(\varepsilon)^{c}\right) & \leqslant 4 m \exp \left(-\frac{\varepsilon^{2} \sigma_{d}^{4}\left(\mathcal{H}_{N}\right) N^{2}}{256 m \Delta^{4}}\right) \\
& \leqslant \exp \left(-\frac{\varepsilon^{2} \sigma_{d}^{4}\left(\mathcal{H}_{N}\right) N^{2}}{512 m \Delta^{4}}\right)
\end{aligned}
$$

where the last inequality holds for large $N$ since $\sigma_{d}\left(\mathcal{H}_{N}\right) / \Delta \gg(t \log N / N)^{1 / 4}$. We are now in position to prove the upper bound. We observe that

$$
\mathbb{P}\left(S_{m} \geqslant \frac{a_{N}}{t^{k-1}(1-t)}\right) \leqslant \mathbb{P}\left(\left\{S_{m} \geqslant \frac{a_{N}}{t^{k-1}(1-t)}\right\} \cap E_{N}^{m}(\varepsilon)\right)+\mathbb{P}\left(\left(E_{N}^{m}(\varepsilon)\right)^{c}\right) .
$$

We bound the first probability by Freedman's inequality with $\alpha=a_{N} /\left(t^{k-1}(1-\right.$ $t)), \beta=(1+\varepsilon) \sigma_{d}^{2}\left(\mathcal{H}_{N}\right) N t /(1-t)$ and $R=C \Delta$ (for some constant $C$ depending 
only on $k$ ), where we use that $\left|X_{1}^{\mathcal{H}_{N}}\left(B_{i}\right)\right|=O_{k}(\Delta)$ and $N /(N-i) \leqslant 2$ for all $1 \leqslant i \leqslant m$. Moreover we bound the second probability by (6-8) and then we obtain

$$
\begin{aligned}
& \mathbb{P}\left(S_{m} \geqslant \frac{a_{N}}{t^{k-1}(1-t)}\right) \leqslant \\
& \exp \left(\frac{-a_{N}^{2}}{2(1+\varepsilon)(1-t) t^{2 k-1} \sigma_{d}^{2}\left(\mathcal{H}_{N}\right) N+2 C \Delta t^{k-1}(1-t) a_{N}}\right)+ \\
& \exp \left(-\frac{\varepsilon^{2} \sigma_{d}^{4}\left(\mathcal{H}_{N}\right) N^{2}}{512 m \Delta^{4}}\right)
\end{aligned}
$$

By the conditions on $a_{N}$, the second term of the denominator in the first exponential is o(1) times the first term and the second exponential is o(1) times the first exponential. Therefore we have

$$
\mathbb{P}\left(S_{m} \geqslant \frac{a_{N}}{t^{k-1}(1-t)}\right) \leqslant \exp \left(\frac{-a_{N}^{2}(1-O(\varepsilon))}{2(1-t) t^{2 k-1} \sigma_{d}^{2}\left(\mathcal{H}_{N}\right) N}\right) .
$$

Since $\varepsilon$ is arbitrary, this gives us the desired upper bound

$$
\mathbb{P}\left(S_{m} \geqslant \frac{a_{N}}{t^{k-1}(1-t)}\right) \leqslant \exp \left(\frac{-(1+o(1)) a_{N}^{2}}{2(1-t) t^{2 k-1} \sigma_{d}^{2}\left(\mathcal{H}_{N}\right) N}\right) .
$$

For the lower bound of (6-7), we need to work slightly differently, because we need to use Lemma 2.16 instead of Lemma 2.15. We fix $0<\varepsilon<1 / 2$ and define $F_{N}^{m}(\varepsilon)$ to be the event that there is $1 \leqslant \ell \leqslant m$ such that

$$
S_{\ell} \geqslant \frac{(1+\varepsilon) a_{N}}{t^{k-1}(1-t)}
$$

Applying Freedman's inequality to the part of the martingale that occurs after first crossing $(1+\varepsilon) a_{N} / t^{k-1}(1-t)$, it is straightforward that (provided $N$ is sufficiently large) there is probability at least $1 / 2$ that the martingale remains above $a_{N} / t^{k-1}(1-t)$. Therefore

$$
\mathbb{P}\left(S_{m} \geqslant \frac{a_{N}}{t^{k-1}(1-t)}\right) \geqslant \frac{1}{2} \mathbb{P}\left(F_{N}^{m}(\varepsilon)\right)
$$

and so it is sufficient to prove that

$$
\mathbb{P}\left(F_{N}^{m}(\varepsilon)\right) \geqslant \exp \left(\frac{-(1+O(\varepsilon)) a_{N}^{2}}{2(1-t) t^{2 k-1} \sigma_{d}^{2}\left(\mathcal{H}_{N}\right) N}\right) .
$$

Setting $\alpha=(1+\varepsilon) a_{N} /\left(t^{k-1}(1-t)\right)$ and $\beta=(1-\varepsilon) \sigma_{d}^{2}\left(\mathcal{H}_{N}\right) N t /(1-t)$, we 
observe that

$$
\left\{T_{\alpha} \leqslant \beta\right\} \subset\left(E_{N}^{m}(\varepsilon)\right)^{c} \cup F_{N}^{m}(\varepsilon),
$$

where we recall that $T_{\alpha}=V\left(m_{\alpha}\right)$ and $m_{\alpha}$ is the least $m$ such that $S_{m}>S_{0}+\alpha$ (we let $m_{\alpha}=\infty$ if there is no such $m$ ). Hence we obtain that

$$
\mathbb{P}\left(F_{N}^{m}(\varepsilon)\right) \geqslant \mathbb{P}\left(T_{\alpha} \leqslant \beta\right)-\mathbb{P}\left(\left(E_{N}^{m}(\varepsilon)\right)^{c}\right) .
$$

By Lemma 2.16 and (6-8), this gives us

$$
\mathbb{P}\left(F_{N}^{m}(\varepsilon)\right) \geqslant \frac{1}{2} \exp \left(\frac{-\alpha^{2}(1+4 \delta)}{2 \beta}\right)-\exp \left(-\frac{\varepsilon^{2} \sigma_{d}^{4}\left(\mathcal{H}_{N}\right) N^{2}}{512 m \Delta^{4}}\right)
$$

where $\delta>0$ is minimal such that $\beta / \alpha \geqslant 9 R \delta^{-2}$ and $\alpha^{2} / \beta \geqslant 16 \delta^{-2} \log \left(64 \delta^{-2}\right)$ (recall that $R=O_{k}(\Delta)$ ). Replacing the values of $\alpha$ and $\beta$ and since the second exponential is $o(1)$ times the first, for sufficiently large $N$, we have

$$
\mathbb{P}\left(F_{N}^{m}(\varepsilon)\right) \geqslant \frac{1}{4} \exp \left(\frac{-(1+O(\varepsilon)+O(\delta)) a_{N}^{2}}{2(1-t) t^{2 k-1} \sigma_{d}^{2}\left(\mathcal{H}_{N}\right) N}\right) .
$$

Finally, from the conditions imposed on $a_{N}$ (which imply $\alpha R \ll \beta \ll \alpha^{2}$ ), it follows that $\delta=o(1)$. Since $\varepsilon$ is arbitrary, we obtain the lower bound, which completes the proof of the proposition.

\section{6}

\section{Proof of Theorem 6.1}

We are now in position to prove Theorem 6.1, combining Lemma 6.7 with Propositions 6.5 and 6.11. In general lines, we use the following strategy. Fixing $\varepsilon>0$ small, recalling that

$$
D^{\mathcal{H}}\left(B_{m}\right)=\Lambda^{\mathcal{H}}\left(B_{m}\right)+D^{\mathcal{H}, \perp}\left(B_{m}\right)
$$

we have by the union bound

$$
\begin{aligned}
& \mathbb{P}\left(D^{\mathcal{H}}\left(B_{m}\right) \geqslant a_{N}\right) \leqslant \mathbb{P}\left(\Lambda^{\mathcal{H}, *}\left(B_{m}\right) \geqslant(1-2 \varepsilon) a_{N}\right)+ \\
& \mathbb{P}\left(\Lambda^{\mathcal{H}}\left(B_{m}\right)-\Lambda^{\mathcal{H}, *}\left(B_{m}\right) \geqslant \varepsilon a_{N}\right)+\mathbb{P}\left(D^{\mathcal{H}, \perp}\left(B_{m}\right) \geqslant \varepsilon a_{N}\right) .
\end{aligned}
$$

We then use Proposition 6.11 to control the first probability and Lemma 6.7 and Proposition 6.5 to ensure that the last two probabilities are very small compared to the first one. This gives an upper bound for $\mathbb{P}\left(D^{\mathcal{H}}\left(B_{m}\right) \geqslant a_{N}\right)$ and the lower bound follows in a similar way. 
Proof of Theorem 1.7. First we fix $0<\varepsilon<1 / 100$. Then Proposition 6.11 gives us

$$
\mathbb{P}\left(\Lambda^{\mathcal{H}, *}\left(B_{m}\right) \geqslant(1-2 \varepsilon) a_{N}\right) \leqslant \exp \left(\frac{-(1-5 \varepsilon) a_{N}^{2}}{2(1-t) t^{2 k-1} \sigma_{d}^{2}\left(\mathcal{H}_{N}\right) N}\right)
$$

Since $a_{N} \gg t \Delta$ and $\left|\Lambda^{\mathcal{H}}\left(B_{m}\right)-\Lambda^{\mathcal{H}, *}\left(B_{m}\right)\right|=O_{k}(t \Delta)$ by Lemma 6.7 , we have that

$$
\mathbb{P}\left(\Lambda^{\mathcal{H}}\left(B_{m}\right)-\Lambda^{\mathcal{H}, *}\left(B_{m}\right) \geqslant \varepsilon a_{N}\right)=0
$$

for sufficiently large $N$. Furthermore, applying Proposition 6.5 with $\theta=$ $(1-5 \varepsilon) /\left[2(1-t) \varepsilon^{2}\right] \geqslant 1$, since $\varepsilon<1 / 100$, we obtain

$$
\begin{aligned}
& \mathbb{P}\left(D^{\mathcal{H}, \perp}\left(B_{m}\right) \geqslant \varepsilon a_{N}\right) \leqslant \exp \left(\frac{-(1-5 \varepsilon) a_{N}^{2}}{2(1-t) t^{2 k-1} \sigma_{d}^{2}\left(\mathcal{H}_{N}\right) N}\right)+ \\
& N^{O_{k}(1)} \exp \left(\frac{-\Omega_{k, \Delta_{2}}(1) t^{k-1} \varepsilon^{2} \sigma_{d}^{2}(\mathcal{H})}{(1-5 \varepsilon) \Delta}\right)
\end{aligned}
$$

where we used $2(1-t) \geqslant 1$ in the second exponential. Since $\Delta_{2}\left(\mathcal{H}_{N}\right)=O(1)$ and $\sigma_{d}^{2}\left(\mathcal{H}_{N}\right) / \Delta \gg \log N / t^{k-1}$, there exists a constant $C=C(k)>0$ such that

$$
\begin{aligned}
& \mathbb{P}\left(D^{\mathcal{H}, \perp}\left(B_{m}\right) \geqslant \varepsilon a_{N}\right) \leqslant \exp \left(\frac{-(1-5 \varepsilon) a_{N}^{2}}{2(1-t) t^{2 k-1} \sigma_{d}^{2}\left(\mathcal{H}_{N}\right) N}\right)+ \\
& \exp \left(\frac{-C t^{k-1} \varepsilon^{2} \sigma_{d}^{2}(\mathcal{H})}{(1-5 \varepsilon) \Delta}\right) .
\end{aligned}
$$

Since

$$
a_{N} \ll \frac{t^{3 k / 2-1} \sigma_{d}^{2}\left(\mathcal{H}_{N}\right) N^{1 / 2}}{\Delta^{1 / 2}},
$$

the second exponential in the above expression is $o(1)$ times the first and therefore we get

$$
\mathbb{P}\left(D^{\mathcal{H}, \perp}\left(B_{m}\right) \geqslant \varepsilon a_{N}\right) \leqslant(1+\varepsilon) \exp \left(\frac{-(1-5 \varepsilon) a_{N}^{2}}{2(1-t) t^{2 k-1} \sigma_{d}^{2}\left(\mathcal{H}_{N}\right) N}\right) .
$$

Replacing (6-10), (6-11) and (6-12) in (6-9) and recalling that $a_{N} \gg$ $t^{k-1 / 2} \sigma_{d}^{2}\left(\mathcal{H}_{N}\right) N^{1 / 2}$, we obtain

$$
\mathbb{P}\left(D^{\mathcal{H}}\left(B_{m}\right) \geqslant \varepsilon a_{N}\right) \leqslant \exp \left(\frac{-(1-6 \varepsilon) a_{N}^{2}}{2(1-t) t^{2 k-1} \sigma_{d}^{2}\left(\mathcal{H}_{N}\right) N}\right) .
$$

As $\varepsilon$ is arbitrary, this gives us

$$
\mathbb{P}\left(D^{\mathcal{H}}\left(B_{m}\right) \geqslant \varepsilon a_{N}\right) \leqslant \exp \left(\frac{-(1+o(1)) a_{N}^{2}}{2(1-t) t^{2 k-1} \sigma_{d}^{2}\left(\mathcal{H}_{N}\right) N}\right) .
$$


The lower bound follows by the same argument and the inequality

$$
\begin{aligned}
& \mathbb{P}\left(D^{\mathcal{H}}\left(B_{m}\right) \geqslant a_{N}\right) \geqslant \mathbb{P}\left(\Lambda^{\mathcal{H}, *}\left(B_{m}\right) \geqslant(1+2 \varepsilon) a_{N}\right)- \\
& \mathbb{P}\left(\Lambda^{\mathcal{H}, *}\left(B_{m}\right)-\Lambda^{\mathcal{H}}\left(B_{m}\right) \geqslant \varepsilon a_{N}\right)-\mathbb{P}\left(D^{\mathcal{H}, \perp}\left(B_{m}\right) \leqslant-\varepsilon a_{N}\right) .
\end{aligned}
$$




\section{7}

\section{Deviations in p-model for non-regular hypergraphs}

In this chapter, we prove Theorem 1.8, which we restate below for the convenience of the reader.

Theorem (Restatement of Theorem 1.8) Let $\mathcal{H}_{N}$ be a sequence of $k$ uniform hypergraphs $(k \geqslant 2)$ such that $V\left(\mathcal{H}_{N}\right)=[N]$ with $e\left(\mathcal{H}_{N}\right)=\Theta\left(N^{2}\right)$, maximum pair degree $\Delta_{2}=O(1)$ and $\sigma_{d}\left(\mathcal{H}_{N}\right)=\Theta(N)$. Let $p=p_{N}$, bounded away from $1 / 2^{1}$, be such that $p \gg(\log N / N)^{1 /(k-1)}$ and let $\delta_{N}$ be a sequence such that

$$
\max \left\{\frac{1}{p^{k-1} N}, \frac{1}{\sqrt{p N}}\right\} \ll \delta_{N} \ll p^{k / 2-1} .
$$

Then

$$
\mathbb{P}\left(D^{\mathcal{H}_{N}}\left(B_{p}\right) \geqslant \delta_{N} L^{\mathcal{H}_{N}}(p)\right)=\exp \left(\frac{-(1+o(1)) \delta_{N}^{2} p e\left(\mathcal{H}_{N}\right)^{2}}{2(1-p)\left(\bar{d}\left(\mathcal{H}_{N}\right)^{2}+\sigma_{d}^{2}\left(\mathcal{H}_{N}\right)\right) N}+O(\log N)\right) .
$$

To streamline notation we simply write $\sigma$ for $\sigma_{d}\left(\mathcal{H}_{N}\right), \bar{d}$ for $\bar{d}\left(\mathcal{H}_{N}\right)$ and $h$ for $e\left(\mathcal{H}_{N}\right)$ throughout this chapter. We recall that if we condition that $B_{p}$ contain exactly $m$ elements then it is distributed as $B_{m}$, and so we have

$$
\mathbb{P}\left(D^{\mathcal{H}_{N}}\left(B_{p}\right) \geqslant \delta_{N} L^{\mathcal{H}_{N}}(p)\right)=\sum_{m=0}^{N} b_{N, p}(m) \mathbb{P}\left(N^{\mathcal{H}_{N}}\left(B_{m}\right) \geqslant\left(1+\delta_{N}\right) p^{k} h\right),
$$

where we used $L^{\mathcal{H}_{N}}(p)=p^{k} h$ and $b_{N, p}(m):=\mathbb{P}(\operatorname{Bin}(N, p)=m)$. As in Chapter 5 , it is useful to have an estimate for $b_{N, p}(m)$. Setting $q:=1-p$, we use the following bound, which is a consequence of Theorem 2.17 .

$$
b_{N, p}(m)=\exp \left(-\frac{(1+o(1)) x(m)^{2}}{2}+O(\log N)\right),
$$

where $x(m)=(m-p N) / \sqrt{p q N}$, which holds provided $N^{-1} \leqslant p \leqslant 1 / 2$, and $\sqrt{p N} \ll m-p N \ll p N$.

We must also consider what deviation is required in the $m$-model for each value of $m$. For $\eta \in[0,1]$ let us define

$$
m_{\eta}:=\left(1+\frac{\eta \delta_{N}}{k}\right) p N
$$

${ }^{1}$ This means that there exists a constant $\varepsilon>0$ such that $p \leqslant 1 / 2-\varepsilon$. 
We may think of (7-1) as offering us various ways to achieve the required deviation. The term $m=m_{0}$ corresponds to a case where the number of points in $B_{p}$ is equal to its expected value, $p N$, and all the work of achieving the deviation must be done in the $m$-model. On the other hand $m=m_{1}$ corresponds to a large enough deviation in the number of points in $B_{p}$ that no (significant) deviation is required in the $m$-model, as $L^{\mathcal{H}_{N}}\left(m_{1}\right) \approx\left(1+\delta_{N}\right) h$. So we will be interested in the choice of $\eta \in[0,1]$ which minimises the total "cost" of the deviation. In fact this will be achieved by

$$
\eta^{*}:=\frac{\bar{d}^{2}}{\bar{d}^{2}+\sigma^{2}}
$$

Note that for $\eta \in[0,1]$ we have by a fairly simple computation

$$
L^{\mathcal{H}_{N}}\left(m_{\eta}\right)=p^{k} h+(1+o(1)) \eta \delta_{N} p^{k} h+O\left(p^{k-1} h / N\right) .
$$

Therefore, achieving the deviation $N^{\mathcal{H}_{N}}\left(m_{\eta}\right) \geqslant\left(1+\delta_{N}\right) p^{k} h$ corresponds to

$$
D^{\mathcal{H}_{N}}\left(m_{\eta}\right) \geqslant(1-\eta+o(1)) \delta_{N} p^{k} h
$$

since $p^{k-1} h / N=o\left(\delta_{N} p^{k} h\right)$. We are now ready to prove Theorem 1.8.

Proof of Theorem 1.8. Since we have a $O(\log N)$ as an error term, which is equivalent to a multiplicative $N^{O(1)}$, it suffices to prove a result for the maximum contribution (as there are only $N$ values of $m$ ) in (7-1). In other words we must prove that

$$
\max _{m} f(m)=\exp \left(\frac{-(1+o(1)) \delta_{N}^{2} p h^{2}}{2 q\left(\bar{d}^{2}+\sigma^{2}\right) N}+O(\log N)\right),
$$

where

$$
f(m):=b_{N, p}(m) \mathbb{P}\left(N^{\mathcal{H}_{N}}\left(B_{m}\right) \geqslant\left(1+\delta_{N}\right) p^{k} h\right) .
$$

We consider three regimes of $m$ : (i) $m \leqslant m_{0}$, (ii) $m_{0} \leqslant m \leqslant m_{1}$ and (iii) $m \geqslant m_{1}$.

In the first case, since $b_{N, p}(m) \leqslant 1$ and as the event $N^{\mathcal{H}_{N}}\left(B_{m}\right) \geqslant$ $\left(1+\delta_{N}\right) p^{k} h$ is increasing in $m$, we have

$$
\begin{aligned}
f(m) & \left.\leqslant \mathbb{P}\left(N^{\mathcal{H}_{N}}\left(B_{m_{0}}\right)\right) \geqslant\left(1+\delta_{N}\right) p^{k} h\right) \\
& =\mathbb{P}\left(D^{\mathcal{H}_{N}}\left(B_{m_{0}}\right) \geqslant(1+o(1)) \delta_{N} p^{k} h\right),
\end{aligned}
$$

where we used (7-3) in the last line. The conditions on $\delta_{N}$ allow us to apply 
Theorem 1.7 and so we obtain

$$
\begin{aligned}
f(m) & \leqslant \exp \left(\frac{-(1+o(1)) \delta_{N}^{2} p h^{2}}{2 q \sigma^{2} N}\right) \\
& \leqslant \exp \left(\frac{-(1+o(1)) \delta_{N}^{2} p h^{2}}{2 q\left(\bar{d}^{2}+\sigma^{2}\right) N}+O(\log N)\right) .
\end{aligned}
$$

In the third case, since $\mathbb{P}\left(N^{\mathcal{H}_{N}}\left(B_{m}\right) \geqslant\left(1+\delta_{N}\right) p^{k} h\right) \leqslant 1$ and $b_{N, p}(m) \leqslant$ $b_{N, p}\left(m_{1}\right)$ for $m \geqslant m_{1}$, we obtain

$$
\begin{aligned}
f(m) & \leqslant b_{N, p}\left(m_{1}\right) \\
& =\exp \left(-(1+o(1)) \frac{\delta_{N}^{2} p N}{2 q k^{2}}+O(\log N)\right) \\
& \leqslant \exp \left(\frac{-(1+o(1)) \delta_{N}^{2} p h^{2}}{2 q\left(\bar{d}^{2}+\sigma^{2}\right) N}+O(\log N)\right),
\end{aligned}
$$

where we used in the second line the estimate for $b_{N, p}(m)$ given in (7-2).

In the third case, we must take into account the contributions from both the binomial distribution and the deviations in the $m$-model. Since $m_{0} \leqslant m \leqslant m_{1}$, it suffices to prove

$$
\max _{\eta \in[0,1]} f\left(m_{\eta}\right)=\exp \left(\frac{-(1+o(1)) \delta_{N}^{2} p h^{2}}{2 q\left(\bar{d}^{2}+\sigma^{2}\right) N}+O(\log N)\right) .
$$

The corresponding value of $x, x\left(m_{\eta}\right)$, is

$$
x\left(m_{\eta}\right)=\frac{\eta \delta_{N} p^{1 / 2} N^{1 / 2}}{k q^{1 / 2}} .
$$

Using (7-2), it follows that

$$
b_{N}\left(m_{\eta}\right)=\exp \left(-(1+o(1)) \frac{\eta^{2} \delta_{N}^{2} p N}{2 q k^{2}}+O(\log N)\right)
$$

On the other hand, by (7-3) and Theorem 1.7, we have

$$
\begin{aligned}
\mathbb{P}\left(N^{\mathcal{H}_{N}}\left(m_{\eta}\right) \geqslant\left(1+\delta_{N}\right) h\right) & =\mathbb{P}\left(D^{\mathcal{H}_{N}}\left(m_{\eta}\right) \geqslant(1-\eta+o(1)) \delta_{N} p^{k} h\right) \\
& =\exp \left(\frac{-(1+o(1))(1-\eta)^{2} \delta_{N}^{2} p h^{2}}{2 q \sigma^{2} N}\right) \\
& =\exp \left(\frac{-(1+o(1))(1-\eta)^{2} \delta_{N}^{2} p \bar{d}^{2} N}{2 q k^{2} \sigma^{2}}\right)
\end{aligned}
$$

where we have used in the final line that $h=\bar{d} N / k$, which is true by the 
handshaking lemma (Lemma 2.1). It follows that

$$
f\left(m_{\eta}\right)=\exp \left(\frac{-(1+o(1)) \delta_{N}^{2} p N}{2 q k^{2} \sigma^{2}}\left[\sigma^{2} \eta^{2}+\bar{d}^{2}(1-\eta)^{2}\right]\right) .
$$

This expression is maximized by choosing $\eta$ to minimize $\sigma^{2} \eta^{2}+\bar{d}^{2}(1-\eta)^{2}$. This value of $\eta$ is

$$
\eta^{*}=\frac{\bar{d}^{2}}{\bar{d}^{2}+\sigma^{2}}
$$

and in this case (using $\bar{d}=h k / N$ ) we have precisely

$$
f\left(m_{\eta^{*}}\right)=\exp \left(\frac{-(1+o(1)) \delta_{N}^{2} p h^{2}}{2 q\left(\bar{d}^{2}+\sigma^{2}\right) N}+O(\log N)\right)
$$

as required. 


\section{8 \\ Applications for arithmetic configurations in random sets}

In this section we collect some applications of Theorems 1.2, 1.5, 1.7 and 1.8 in order to illustrate their use in obtaining bounds of deviations for the count of arithmetic structures in random sets. The section is divided into two parts. In the first, we consider random sets over the additive group $\mathbb{Z}_{N}$, where $N$ is a prime number. In the second part, we consider random sets over the set of the first $N$ positive integers, $[N]$. The results for regular hypergraphs are well suited for the first case, while the results for non-regular hypergraphs will handle the second case.

\section{1}

\section{Progressions in $\mathbb{Z}_{N}$}

We consider deviations for the $m$-model first. Let $N^{k}\left(B_{m}\right)$ denote the number of $k$-progressions in a random subset $B_{m}$ of the cyclic group $\mathbb{Z} / N \mathbb{Z}$, $N$ prime, and let $D^{k}\left(B_{m}\right)$ be the deviation of $N^{k}\left(B_{m}\right)$.

Define $\mathcal{H}_{k}$ to be the $k$-uniform hypergraph with vertex set $\mathbb{Z} / N \mathbb{Z}$ and edges the nontrivial $k$-progressions $\{x, x+d, \ldots, x+(k-1) d\}$ with $x \in \mathbb{Z} / N \mathbb{Z}$ and $d \in\{1,2, \ldots,(N-1) / 2\}$. Every pair $\{x, y\}$ of elements in $\mathbb{Z} / N \mathbb{Z}$ is contained in $\left(\begin{array}{l}k \\ 2\end{array}\right)$ edges of $\mathcal{H}_{k}$, so that the hypergraph is 2 -regular and hence 1-regular. A direct application of Theorem 1.1 for $r=2$ gives in this case,

Theorem 8.1 For each $a>0$,

$$
\mathbb{P}\left(D^{k}\left(B_{m}\right)>a\right) \leqslant N^{c_{1}} \exp \left(-c_{2} \frac{a}{m}\right)
$$

for some constants $c_{1}, c_{2}$ which depend only on $k$. The same inequality holds for negative deviations.

The expected value of $N^{k}\left(B_{m}\right)$ in this example is

$$
L^{k}(m)=\left(\begin{array}{c}
N \\
2
\end{array}\right) \frac{(m)_{k}}{(N)_{k}} \sim \frac{m^{k}}{2 N^{k-2}} .
$$

Bounds on moderate deviations are thus obtained in Theorem 8.1 for $a \ll$ $m^{k} / N^{k-2}$. In particular, for $m \log N \ll a \ll m^{k} / N^{k-2}$ we obtain exponentially small bounds for moderate deviations of the $k$-progressions count, which apply 
to random sets of $\mathbb{Z} / N \mathbb{Z}$ of size $m \gg\left(N^{k-2} \log N\right)^{1 /(k-1)}$. This is slightly above the threshold cardinality for the existence of $k$-progressions in a random set (see Rödl, Ruciński [28]). One can not expect to obtain such small deviation for smaller sets since, as shown in Rué, Spiegel and Zumalacárregui [29], the count of $k$-progressions within the threshold window converges to a Poisson distribution.

We note that the term in the exponential in Theorem 1.1 for $r=3$ corresponding to this example would be $-\Omega_{k}(1) a^{2 / 3} / m$ (as $\Delta_{3}=O_{k}(1)$ ), while for $r=1$ we have $\Delta_{1}=\Theta_{k}(N)$ and we would obtain $-\Omega_{k}(1) a^{2} / m N$. Both are usually worse than the one obtained for $r=2$ as displayed in the bound of Theorem 8.1. This exemplifies Remark 1.4 in the Introduction.

The bounds on deviations for the counting in the $m$-model can be transferred to the $p$-binomial model using Theorem 1.5. Taking into account the 1-regulariy of the hypergraph $\mathcal{H}_{k}$, Theorem 1.5 with $\eta=0$ and $r=2$ (recalling that $e\left(\mathcal{H}_{k}\right)=\left(\begin{array}{c}N \\ 2\end{array}\right)$ and $\Delta_{2}\left(\mathcal{H}_{k}\right)=\left(\begin{array}{c}k \\ 2\end{array}\right)$ ) gives us

Theorem 8.2 Let $D^{k}\left(B_{p}\right)$ denote the deviation of the $k$-progressions count in a p-random subset $B_{p}$ of $\mathbb{Z} / N \mathbb{Z}, N$ prime.

Let $\delta_{N}$ be a sequence satisfying

$$
\max \left\{\frac{\log N}{p^{k-1} N}, \frac{1}{\sqrt{p N}}\right\} \ll \delta_{N} \ll p^{k-2} .
$$

If $p$ is bounded away from 1 , then

$$
\mathbb{P}\left(D^{k}\left(B_{p}\right)>\delta_{N} L^{k}(p)\right)=\exp \left(-(1+o(1)) \frac{\delta_{N}^{2} p N}{2 k^{2}(1-p)}\right)
$$

Furthermore, the same bounds apply to the corresponding negative deviations.

We observe that Theorem 8.2 provides exponentially small bounds of moderate deviations for $p \gg(\log N / N)^{1 /(2 k-3)}$.

We remark that the above result was obtained by Bhattacharya, Ganguly, Shao and Zhao [21] under the following conditions on $p$ and $\delta_{N}: p \rightarrow 0$, $\delta_{N}=O(1), \delta_{N}^{-3} p^{k-2}(\log (1 / p))^{2} \rightarrow \infty$, and

$$
\min \left\{\delta_{N} p^{k}, \delta_{N}^{2} p\right\} \geqslant N^{-\frac{1}{6(k-1)}} \log N .
$$

For $k=3$, the right-hand side can be relaxed to $N^{-1 / 6}(\log N)^{7 / 6}$ and for $k=4$, it can be relaxed to $N^{-1 / 12}(\log N)^{13 / 12}$. As we have seen in the introduction, our result extends the range of $p$ and $\delta_{N}$ for which the above asymptotic of the log probability holds. 


\section{2 \\ Schur equation in $\mathbb{Z}_{N}$}

In addition to the 3-APs, let us mention another 3-variable case, the Schur equation $x+y=z$, related to problems on sum-free sets. Threshold cardinalities and large deviations for the Schur equation have also been addressed in the literature, see e.g. [20, 30]. Consider the hypergraph $\mathcal{H}$ with vertex set the nonzero elements of $\mathbb{Z} / N \mathbb{Z}$ and edge set the pairwise distinct Schur triples $\{x, y, z\}$ with $x+y=z$, none of them zero. Every nonzero element $x$ in $\mathbb{Z} / N \mathbb{Z}$ belongs to $N-3$ triples of the form $\{x, y, x+y\}$ with $y \notin\{0, x,-x\}$ and $(N-3) / 2$ triples of the form $\{y, x-y, x\}$ with $y \notin\{0, x, x / 2\}$. Therefore, $\mathcal{H}$ is 1 -regular with $d_{1}(\mathcal{H})=3(N-3) / 2$ and maximum 2 -degree $\Delta_{2}(\mathcal{H})=3$. Let $D^{3}\left(B_{m}\right)$ denote the deviation on the count of Schur triples of nonzero elements in a random set $B_{m}$ of $(\mathbb{Z} / N \mathbb{Z})^{*}$. Therefore Theorem 1.1 for $r=2$ gives us

$$
\mathbb{P}\left(D^{3}\left(B_{m}\right)>a\right)<N^{C} \exp \left(-c\left(\frac{a}{m}\right)\right),
$$

for some constants $c$ and $C$.

The bounds on the deviations can be analogously transferred to the $p$ binomial model, obtaining the same result as in Corollary 1.6.

\section{3 \\ Sidon equation in $\mathbb{Z}_{N}$}

The Sidon equation $x+y=z+t$ has also been treated in the literature, see e.g. [29, 31]. In particular, bounds of the deviation on the number of solutions of the Sidon equation are given in [31, Lemma 5.3] by means of the Kim-Vu polynomial concentration inequality [11]. An analogous analysis can be carried over in our context to obtain bounds on moderate deviations giving more precise results in an appropriate range of the size of random sets. The number of nontrivial quadruples $(x, y, z, t)$ in a set $A \subset \mathbb{Z} / N \mathbb{Z}$ which satisfy the Sidon equation $x+y=z+t$ with $\{x, y\} \neq\{z, t\}$ is also called the Additive Energy of the set (which counts ordered quadruples). For unordered subsets, solutions of the Sidon equation in $A$ are either 3 progressions $\{x, y, z=t=(x+y) / 2\}$, which satisfy $x+y=2 z$, or quadruples $\{x, y, z, t\}$ of distinct elements.

We consider the 4 -uniform hypergraph $\mathcal{H}_{4}$ which has vertex set $\mathbb{Z} / N \mathbb{Z}$ (as usual we consider $N$ prime) and edges the pairwise distinct Sidon quadruples $\{x, y, z, t\}$ with $x+y=z+t$. Every pair $\{x, y\}$ is contained in $(N-3) / 2$ quadruples of the form $\{x, y, z, x+y-z\}$ with $z \notin\{x, y,(x+y) / 2\}$ and $(N-3)$ quadruples of the form $\{x, y, z, x+z-y\}$ with $z \notin\{x, y,(x-y) / 2\}$, so 
that $\mathcal{H}_{4}$ is $2-$ regular with $d_{2}\left(\mathcal{H}_{4}\right)=3(N-3) / 2$. Moreover, each triple $\{x, y, z\}$ which is not in arithmetic progression belongs to three distinct quadruples $\{x, y, z, t\}$ with $t \in\{x+y-z, x+z-y, y+z-x\}$, while triples which are 3 -progressions, say $z=(x+y) / 2$, belong to two distinct quadruples. Hence it follows that $\Delta_{3}\left(\mathcal{H}_{4}\right)=3$. By denoting by $D^{4}\left(B_{m}\right)$ the deviation in the count of Sidon quadruples of pairwise distinct elements, Theorem 1.1 with $r=3$ gives, for all $a>0$,

$$
\mathbb{P}\left(D^{4}\left(B_{m}\right)>a\right) \leqslant N^{c_{1}} \exp \left(-c_{2} \frac{a^{2 / 3}}{m}\right),
$$

for some constants $c_{1}, c_{2}$. By bounding the deviation $D^{S}\left(B_{m}\right)$ on the count of the number of solutions of the Sidon equation by the sum of deviations on the count of additive quadruples of distinct elements and the count of 3progressions from Theorem 8.1 we obtain

$$
\mathbb{P}\left(D^{S}\left(B_{m}\right)>a\right) \leqslant N^{c_{3}} \exp \left(-c_{S} \frac{a^{2 / 3}}{m}\right)
$$

for some constants $c_{3}$ and $c_{S}$. The expected number of solutions of the Sidon equation in a random set $B_{m}$ is

$$
L^{S}(m)=\frac{(m)_{3}}{2(N-2)}+\frac{(m)_{4}}{8(N-2)}
$$

Therefore, for $a \ll m^{4} / N$ and $a^{2 / 3} \gg m \log N$ we obtain exponentially small bounds for the deviation in the count of solutions of the Sidon equation, which apply to random sets of cardinality $m \gg N^{2 / 5}(\log N)^{3 / 5}$. This shows that, for these values of $m$, the Additive Energy of a random $m$-set in $\mathbb{Z} / N \mathbb{Z}$ is highly concentrated on its mean value. We observe that in this case the threshold for the appearance of solutions of the Sidon equation is $N^{1 / 4}$ and our bound starts to be effective only above $N^{2 / 5}$.

As in the preceding examples, Theorem 1.5 can be applied to transfer the bounds to the $p$-binomial model. Let $D^{4}\left(B_{p}\right)$ denote the deviation on the number of solutions to the Sidon equation with pairwise distinct entries in a random set $B_{p}$ of $\mathbb{Z} / N \mathbb{Z}$ and $L^{4}(p)$ its mean value. Taking into account that the hypergraph $\mathcal{H}_{4}$ is 2-regular, has constant maximum degree $\Delta_{3}\left(\mathcal{H}_{4}\right)=3$, and has $\Theta\left(N^{3}\right)$ edges, Theorem 1.5 with $r=3$ and $\eta=0$ gives

$$
\mathbb{P}\left(D^{4}\left(B_{p}\right)>\delta_{N} L^{4}(p)\right)=\exp \left(-(1+o(1)) \frac{\delta_{N}^{2} p N}{32(1-p)}\right),
$$


for every sequence $\delta_{N}$ satisfying

$$
\max \left\{\frac{1}{p^{5 / 2}}\left(\frac{\log N}{N}\right)^{3 / 2}, \frac{1}{\sqrt{p N}}\right\} \ll \delta_{N} \ll p^{1 / 2} .
$$

The above condition on $\delta_{N}$ gives a meaningful range of applications when $p \gg(\log N / N)^{1 / 2}$.

\section{4}

\section{Schur equation in $[N]$}

We denote by $\mathcal{H}^{S c h}$ the hypergraph with vertex set $[N]$ and edges being pairwise distinct solutions of the Schur equation $x+y=z$. In order to apply Theorems 1.7 and 1.8 , we first determine the asymptotics of the average degree and of the variance of the degree sequence of $\mathcal{H}^{\text {Sch }}$.

Lemma 8.3 Consider the hypergraph $\mathcal{H}^{\text {Sch }}$ of pairwise distinct Schur triples in $[N]$. We have

$$
\begin{aligned}
& \bar{d}\left(\mathcal{H}^{S c h}\right)=(1+o(1)) \frac{3 N}{4} . \\
& \sigma_{d}^{2}\left(\mathcal{H}^{S c h}\right)=(1+o(1)) \frac{N^{2}}{48}
\end{aligned}
$$

Proof. For any $a \in[N]$, we have

$$
d_{\mathcal{H}^{S c h}}(a)=\left\lfloor\frac{a-1}{2}\right\rfloor+\min \{a-1, N-a\}+(N-2 a)_{+},
$$

where $(\cdot)_{+}=\max \{\cdot, 0\}$ denotes the positive part, and the three terms account for Schur triples with largest element $a$, middle element $a$, and smallest element $a$, respectively. In particular, this gives us

$$
d_{\mathcal{H}^{\text {Sch }}}(a)=N-a / 2+O(1) .
$$

Then it follows that

$$
\begin{aligned}
\sum_{a \in[N]} d_{\mathcal{H}^{S c h}}(a) & =N^{2}-\frac{N^{2}}{4}+O(N) \\
& =\frac{3 N^{2}}{4}+O(N),
\end{aligned}
$$

which gives us

$$
\bar{d}\left(\mathcal{H}^{S c h}\right)=(1+o(1)) \frac{3 N}{4} .
$$


Moreover,

$$
\begin{aligned}
\sum_{a \in[N]} d_{\mathcal{H}^{\text {Sch }}}(a)^{2} & =\sum_{a \in[N]}\left(N^{2}-a N+\frac{a^{2}}{4}+O(N)\right) \\
& =N^{3}-\frac{N^{3}}{2}+\frac{N^{3}}{12}+O\left(N^{2}\right) \\
& =\frac{7 N^{3}}{12}+O\left(N^{2}\right),
\end{aligned}
$$

where we used the following results

$$
\begin{aligned}
\sum_{a \in[N]} a & =\frac{N^{2}}{2}+O(N), \\
\sum_{a \in[N]} a^{2} & =\frac{N^{3}}{3}+O\left(N^{2}\right) .
\end{aligned}
$$

Therefore the degree variance of $\mathcal{H}^{\text {Sch }}$ is

$$
\begin{aligned}
\sigma_{d}^{2}\left(\mathcal{H}^{S c h}\right) & =\frac{\sum_{a \in[N]} d_{\mathcal{H}^{S c h}}(a)^{2}}{N}-\left(\frac{\sum_{a \in[N]} d_{\mathcal{H}^{S c h}}(a)}{N}\right)^{2} \\
& =\frac{7 N^{2}}{12}+O(N)-\left(\frac{3 N}{4}+O(1)\right)^{2} \\
& =\frac{7 N^{2}}{12}-\frac{9 N^{2}}{16}+O(N) \\
& =\frac{N^{2}}{48}+O(N),
\end{aligned}
$$

which implies the lemma.

We now observe that $\sigma_{d}\left(\mathcal{H}^{S c h}\right)=\Theta(N), \Delta_{2}\left(\mathcal{H}^{S c h}\right)=O(1)$ and $\Delta\left(\mathcal{H}^{S c h}\right)=\Theta(N)$. Therefore Theorem 1.7 and Lemma 8.3 give us

Theorem 8.4 Let $0 \leqslant m \leqslant N$ be such that $t=m / N$ satisfies

$$
\left(\frac{\log N}{N}\right)^{1 / 2} \ll t \leqslant \frac{1}{2}
$$

Let $a_{N}$ be a sequence such that

$$
\max \left\{t N, t^{5 / 2} N^{3 / 2}\right\} \ll a_{N} \ll t^{7 / 2} N^{2}
$$

Then

$$
\mathbb{P}\left(D^{\mathcal{H}^{S c h}}\left(B_{m}\right) \geqslant a_{N}\right)=\exp \left(\frac{-(24+o(1)) a_{N}^{2}}{(1-t) t^{5} N^{3}}\right)
$$

This gives a meaningful range of applications when $N^{-2 / 5} \ll t \leqslant 1 / 2$. Furthermore we can transfer this result for the $p$-model. Recalling that we 
also have $e\left(\mathcal{H}^{S c h}\right)=\Theta\left(N^{2}\right)$, we can apply Theorem 1.8 and Remark 1.9 to obtain

Theorem 8.5 Let $\delta_{N}$ be a sequence satisfying

$$
\max \left\{\frac{1}{p^{2} N}, \sqrt{\frac{\log N}{p N}}\right\} \ll \delta_{N} \ll p^{1 / 2} .
$$

If $p$ is bounded away from $1 / 2$, then

$$
\mathbb{P}\left(D^{\mathcal{H}^{S c h}}\left(B_{p}\right) \geqslant \delta_{N} L^{\mathcal{H}^{S c h}}(p)\right)=\exp \left(\frac{-(3+o(1)) \delta_{N}^{2} p N}{56(1-p)}\right)
$$

This result is applicable when $p \gg N^{-2 / 5}$ and $p$ is bounded away from $1 / 2$.

\section{5}

\section{Progressions in $[N]$}

We denote by $\mathcal{H}^{k}$ the hypergraph encoding increasing $k$-APs in $[N]$. As in the case of Schur triples, we first need to compute the asymptotics of the average degree and of the variance of the degree sequence of $\mathcal{H}^{k}$. The following computation is essentially the same as in [21].

Lemma 8.6 Consider the hypergraph $\mathcal{H}^{k}$ of increasing $k$-APs in $[N]$. We have

$$
\bar{d}\left(\mathcal{H}^{k}\right)=(1+o(1)) \frac{k N}{2(k-1)}
$$

and

$$
\sigma_{d}^{2}\left(\mathcal{H}^{k}\right)=(1+o(1)) \theta_{k} N^{2}
$$

where

$$
\theta_{k}:=\frac{1}{3(k-1)^{2}}\left(k-\frac{3 k^{2}}{4}+\sum_{1 \leqslant i<j \leqslant k} \frac{(k-1)^{2}-(k-j)^{2}-(i-1)^{2}}{(j-1)(k-i)}\right) .
$$

Proof. In the following we will use the convention that $x / 0=+\infty$ and $\min \{x,+\infty\}=x$. For each $a \in[N]$, the number of $k$-APs with $a$ as the $j$-th term is

$$
\min \left\{\left\lfloor\frac{a-1}{j-1}\right\rfloor,\left\lfloor\frac{N-a}{k-j}\right\rfloor\right\}
$$


Indeed, the $k$-AP is determined by its common difference $d$, which satisfies $a-(j-1) d \geqslant 1$ and $a+(k-j) d \leqslant N$ and this gives us

$$
1 \leqslant d \leqslant \min \left\{\left\lfloor\frac{a-1}{j-1}\right\rfloor,\left\lfloor\frac{N-a}{k-j}\right\rfloor\right\} .
$$

Since $1 \leqslant j \leqslant k$, we obtain that

$$
d_{\mathcal{H}^{k}}(a)=\sum_{j=1}^{k} \min \left\{\left\lfloor\frac{a-1}{j-1}\right\rfloor,\left\lfloor\frac{N-a}{k-j}\right\rfloor\right\}
$$

By Riemann sum, we have

$$
\lim _{N \rightarrow \infty} \frac{\bar{d}\left(\mathcal{H}^{k}\right)}{N}=\sum_{j=1}^{k} \int_{0}^{1} \min \left\{\frac{x}{j-1}, \frac{1-x}{k-j}\right\} d x
$$

Each of the integrands is piecewise-linear, with transition point $x=(j-1) /(k-$ 1 ), and so

$$
\begin{aligned}
\int_{0}^{1} \min \left\{\frac{x}{j-1}, \frac{1-x}{k-j}\right\} d x & =\int_{0}^{\frac{j-1}{k-1}} \frac{x}{j-1} d x+\int_{\frac{j-1}{k-1}}^{1} \frac{1-x}{k-j} d x \\
& =\frac{j-1}{2(k-1)^{2}}+\frac{k-j}{2(k-1)^{2}} \\
& =\frac{1}{2(k-1)} .
\end{aligned}
$$

Therefore

$$
\bar{d}\left(\mathcal{H}^{k}\right)=(1+o(1)) \frac{k N}{2(k-1)},
$$

which completes the first part of the lemma.

For the second part, we first observe that

$$
\begin{aligned}
\lim _{N \rightarrow \infty} \frac{\sigma_{d}^{2}\left(\mathcal{H}^{k}\right)}{N^{2}} & =\lim _{N \rightarrow \infty} \frac{\sum_{a \in[N]} d_{\mathcal{H}^{k}}(a)^{2}}{N^{3}}-\lim _{N \rightarrow \infty} \frac{\bar{d}^{2}\left(\mathcal{H}^{k}\right)}{N^{2}} \\
& =\lim _{N \rightarrow \infty} \frac{\sum_{a \in[N]} d_{\mathcal{H}^{k}}(a)^{2}}{N^{3}}-\frac{k^{2}}{4(k-1)^{2}},
\end{aligned}
$$

where we used the first part of the lemma in the last line. It is sufficient now to prove that

$$
\lim _{N \rightarrow \infty} \frac{\sum_{a \in[N]} d_{\mathcal{H}^{k}}(a)^{2}}{N^{3}}=\frac{k}{3(k-1)^{2}}+\sum_{1 \leqslant i<j \leqslant k} \frac{(k-1)^{2}-(k-j)^{2}-(i-1)^{2}}{3(k-1)^{2}(j-1)(k-i)} .
$$


By Riemann sum, we have

$$
\lim _{N \rightarrow \infty} \frac{\sum_{a \in[N]} d_{\mathcal{H}^{k}}(a)^{2}}{N^{3}}=\sum_{i, j=1}^{k} \int_{0}^{1} \min \left\{\frac{x}{i-1}, \frac{1-x}{k-i}\right\} \min \left\{\frac{x}{j-1}, \frac{1-x}{k-j}\right\} d x .
$$

Analogously to the first part of the lemma, we can compute this integral for $1 \leqslant i \leqslant j \leqslant k$ as

$$
\begin{aligned}
& \int_{0}^{\frac{i-1}{k-1}} \frac{x^{2}}{(i-1)(j-1)} d x+\int_{\frac{i-1}{k-1}}^{\frac{j-1}{k-1}} \frac{(1-x) x}{(k-i)(j-1)} d x+\int_{\frac{j-1}{k-1}}^{1} \frac{(1-x)^{2}}{(k-i)(k-j)} d x \\
& =\frac{(k-1)^{2}-(k-j)^{2}-(i-1)^{2}}{6(k-1)^{2}(j-1)(k-i)} .
\end{aligned}
$$

In particular, for $i=j$, this expression is equal to $\frac{1}{3(k-1)^{2}}$. Altogether, since

$$
\sum_{i, j=1}^{k} f(i, j)=\sum_{i=1}^{k} f(i, i)+2 \sum_{1 \leqslant i<j \leqslant k} f(i, j)
$$

the second part of the lemma follows.

Analogously to the previous section, the hypergraph $\mathcal{H}^{k}$ has maximum degree, average degree and standard deviation of the degree sequence of order $N$. Since the maximum pair degree is bounded by $\left(\begin{array}{l}k \\ 2\end{array}\right)$, we are in position to apply Theorems 1.7 and 1.8 to obtain results in both $m$-model and $p$-model. First, in the $m$-model we have the following

Theorem 8.7 Let $0 \leqslant m \leqslant N$ be such that $t=m / N$ satisfies

$$
\left(\frac{\log N}{N}\right)^{1 /(k-1)} \ll t \leqslant \frac{1}{2}
$$

Let $a_{N}$ be a sequence such that

$$
\max \left\{t N, t^{k-1 / 2} N^{3 / 2}\right\} \ll a_{N} \ll t^{3 k / 2-1} N^{2} .
$$

Then

$$
\mathbb{P}\left(D^{\mathcal{H}^{k}}\left(B_{m}\right) \geqslant a_{N}\right)=\exp \left(\frac{-(1+o(1)) a_{N}^{2}}{2 \theta_{k}(1-t) t^{2 k-1} N^{3}}\right),
$$

where $\theta_{k}$ is defined in (8-1).

Concerning the $p$-model, we first define

$$
\gamma_{k}=\frac{4}{3}\left(k+\sum_{1 \leqslant i<j \leqslant k} \frac{(k-1)^{2}-(k-j)^{2}-(i-1)^{2}}{(j-1)(k-i)}\right)
$$


as in $[21]$ so that

$$
\frac{\bar{d}\left(\mathcal{H}^{k}\right)^{2}+\sigma_{d}^{2}\left(\mathcal{H}^{k}\right)}{e\left(\mathcal{H}^{k}\right)^{2}}=(1+o(1)) \frac{\gamma_{k}}{N^{2}}
$$

Hence Theorem 1.8 and Remark 1.9 give the following result.

Theorem 8.8 Let $\delta_{N}$ be a sequence satisfying

$$
\max \left\{\frac{1}{p^{k-1} N}, \sqrt{\frac{\log N}{p N}}\right\} \ll \delta_{N} \ll p^{k / 2-1} .
$$

If $p$ is bounded away from $1 / 2$, then

$$
\mathbb{P}\left(D^{\mathcal{H}^{k}}\left(B_{p}\right) \geqslant \delta_{N} L^{\mathcal{H}^{k}}(p)\right)=\exp \left(\frac{-(1+o(1)) \delta_{N}^{2} p N}{2 \gamma_{k}(1-p)}\right) .
$$

The above result is applicable when $p \gg N^{-2 /(3 k-4)}$. We remark that the above result was obtained by Bhattacharya, Ganguly, Shao and Zhao [21] under the following conditions on $p$ and $\delta_{N}: p \rightarrow 0, \delta_{N}=O(1), \delta_{N}^{-3} p^{k-2}(\log (1 / p))^{2} \rightarrow \infty$, and

$$
\min \left\{\delta_{N} p^{k}, \delta_{N}^{2} p\right\} \geqslant N^{-\frac{1}{6(k-1)}} \log N .
$$

For $k=3$, the right-hand side can be relaxed to $N^{-1 / 6}(\log N)^{7 / 6}$ and for $k=4$, it can be relaxed to $N^{-1 / 12}(\log N)^{13 / 12}$. As we saw in the introduction, our theorem extends the range of $p$ and $\delta_{N}$ for which the result is valid. 


\section{Bibliography}

[1] VAN DER WAERDEN, B. L.. Beweis einer Baudetschen Vermutung. Nieuw Arch. Wisk., 15:212-216, 1927.

[2] ERDŐS, P.; TURÁN, P.. On some sequences of integers. Journal of the London Mathematical Society, s1-11(4):261-264, 1936.

[3] ROTH, K.. On certains sets of integers. Journal of the London Mathematical Society, 28:104-109, 1953.

[4] SZEMERÉDI, E.. On sets of integers containing no four elements in arithmetic progression. Acta Mathematica Academiae Scientiarum Hungarica, 20:89-104, 1969.

[5] SZEMERÉDI, E.. On sets of integers containing no $k$ elements in arithmetic progression. Acta Arithmetica, 27:199-245, 1975.

[6] GREEN, B.; TAO, T.. The primes contain arbitrarily long arithmetic progressions. Annals of Mathematics, 167(2):481-547, 2008.

[7] RUCIŃSKI, A.. When are small subgraphs of a random graph normally distributed? Probability Theory and Related Fields, 78:1-10, 1988.

[8] BARBOUR, A. D.; KAROŃSKI, M. ; RUCIŃSKI, A.. A central limit theorem for decomposable random variables with applications to random graphs. Journal of Combinatorial Theory, Series B, 47(2):125145, 1989.

[9] REINERT, G.; RÖLLIN, A.. Random subgraph counts and $U$ statistics: multivariate normal approximation via exchangeable pairs and embedding. Journal of Applied Probability, 47(2):378-393, 2010.

[10] KROKOWSKI, K.; REICHENBACHS, A. ; THÄLE, C.. Discrete malliavin-stein method: Berry-Esseen bounds for random graphs and percolation. Annals of Probability, 45(2):1071-1109, 2017. 
[11] KIM, J. H.; VU, V. H.. Concentration of multivariate polynomials and its applications. Combinatorica, 20(3):417-434, 2000.

[12] CHATTERJEE, S.; VARADHAN, S. R. S.. The large deviation principle for the Erdős-Rényi random graph. European Journal of Combinatorics, 32(7):1000-1017, 2011.

[13] LUBETZKY, E.; ZHAO, Y.. On the variational problem for upper tails in sparse random graphs. Random Structures and Algorithms, 50(3):420-436, 2017.

[14] CHATTERJEE, S.. An introduction to large deviations for random graphs. Bulletin of the American Mathematical Society, 53(4):617-642, 2016.

[15] HAREL, M.; MOUSSET, F. ; SAMOTIJ, W.. Upper tails via high moments and entropic stability. arXiv:1904.08212, 2019.

[16] DÖRING, H.; EICHELSBACHER, P.. Moderate deviations in a random graph and for the spectrum of bernoulli random matrices. Electronic Journal of Probability, 14:2636-2656, 2009.

[17] DÖRING, H.; EICHELSBACHER, P.. Moderate deviations via cumulants. Journal of Theoretical Probability, 26:360-385, 2013.

[18] GOLDSCHMIDT, C.; GRIFFITHS, S. ; SCOTT, A.. Moderate deviations of subgraph counts in the Erdős-Rényi random graphs $G(n, m)$ and $G(n, p)$. arXiv:1902.06830, 2019.

[19] JANSON, S.; RUCIŃSKI, A.. Upper tails for counting objects in randomly induced subhypergraphs and rooted random graphs. Arkiv för Matematik, 49:79-96, 2011.

[20] WARNKE, L.. Upper tails for arithmetic progressions in random subsets. Israel Journal of Mathematics, 221:317-365, 2017.

[21] BHATTACHARYA, B. B.; GANGULY, S.; SHAO, X. ; ZHAO, Y.. Upper tail large deviations for arithmetic progressions in a random set. International Mathematical Research Notes, 2020(1):167-213, 2020.

[22] FIZ PONTIVEROS, G.; GRIFFITHS, S.; SECCO, M. ; SERRA, O.. Deviation probabilities for arithmetic progressions and other regular discrete structures. arXiv:1910.12835, 2019. 
[23] WILLIAMS, D.. Probability with Martingales. Cambridge Mathematical Textbooks, 1991.

[24] AZUMA, K.. Weighted sums of certain dependent random variables. Tohoku Mathematical Journal, 19(3):357-367, 1967.

[25] HOEFFDING, W.. Probability inequalities for sums of bounded random variables. Journal of the American Statistical Association, 58(301):13-30, 1963.

[26] FREEDMAN, D. A.. On tail probabilities for martingales. The Annals of Probability, 3(1):100-118, 1975.

[27] BAHADUR, R. R.. Some approximations to the binomial distribution function. The Annals of Mathematical Statistics, 31(1):43-54, 1960.

[28] RÖDL, V.; RUCIŃSKI, A.. Rado partition theorem for random subsets of integers. Proc. London Math. Soc., 74:481-502, 1997.

[29] RUÉ, J.; SPIEGEL, C. ; ZUMALACÁRREGUI, A.. Threshold functions and Poisson convergence for systems of equations in random sets. Mathematische Zeitschrift, 288:333-360, 2018.

[30] GRAHAM, R.; RÖDL, V. ; RUCIŃSKI, A.. On Schur properties of random subsets of integers. Journal of Number Theory, 61:388-408, 1996.

[31] KOHAYAKAWA, Y.; LEE, S. J.; RÖDL, V. ; SAMOTIJ, W.. The number of Sidon sets and the maximum size of Sidon sets contained in a sparse random set of integers. Random Structures and Algorithms, 46:1-25, 2015. 